
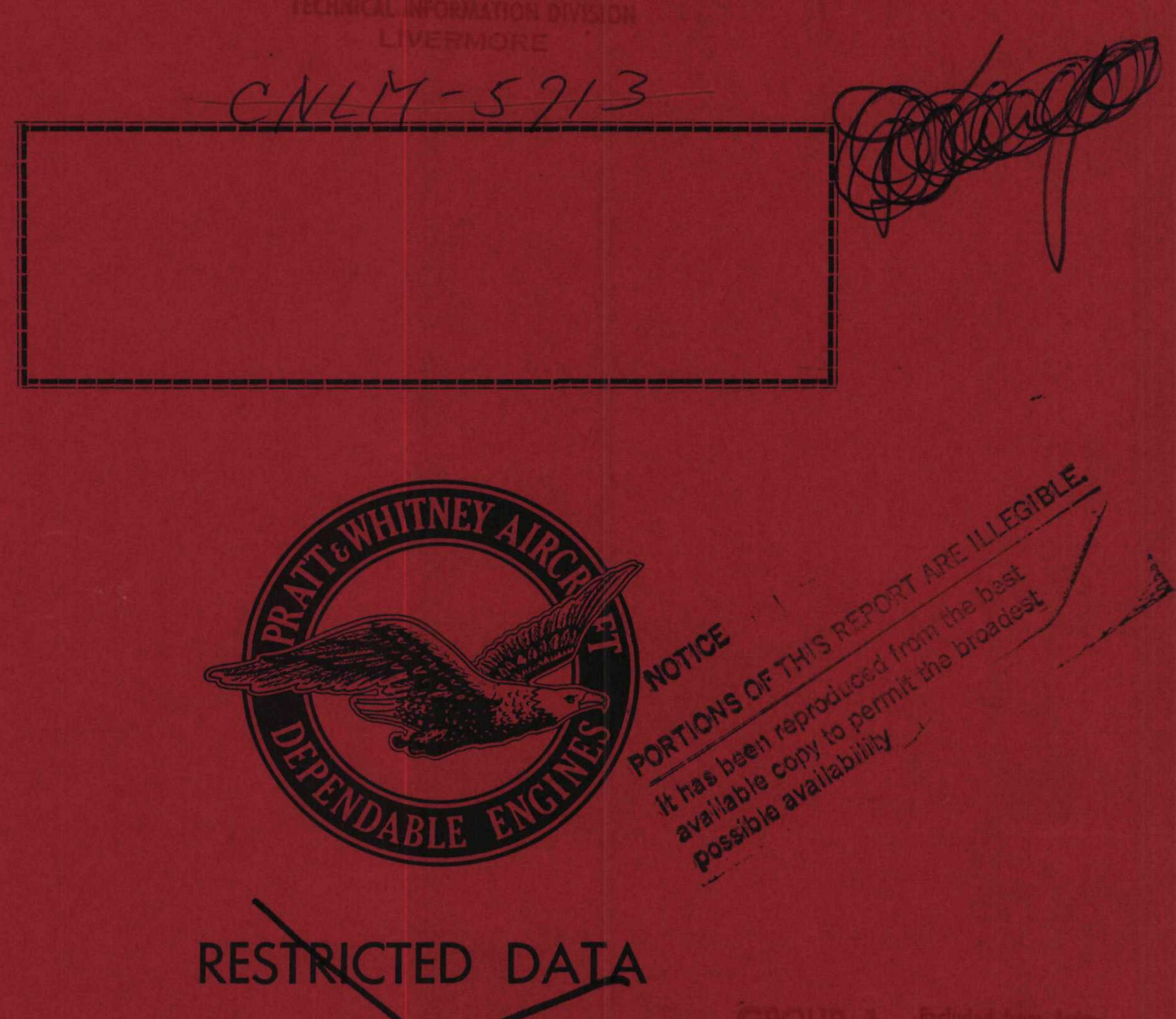

This document contains resticted data as defined in the Atomic Energy Act of 1954. In transmittal or the disclosure of itscontents in any manner te an unauthorized person is prohibited.

$\begin{array}{llllllllllllllllllllll}P & R & A & T & T & \& & W & H & \text { I } & T & N & E & Y & A & \text { I } & R & C & R & A & F & T\end{array}$ DIVISION OF UNITED AIRCRAFT CORPORATION CANEL M I D DLETOWN $\quad$ CONNECTICUT 


\section{DISCLAIMER}

This report was prepared as an account of work sponsored by an agency of the United States Government. Neither the United States Government nor any agency Thereof, nor any of their employees, makes any warranty, express or implied, or assumes any legal liability or responsibility for the accuracy, completeness, or usefulness of any information, apparatus, product, or process disclosed, or represents that its use would not infringe privately owned rights. Reference herein to any specific commercial product, process, or service by trade name, trademark, manufacturer, or otherwise does not necessarily constitute or imply its endorsement, recommendation, or favoring by the United States Government or any agency thereof. The views and opinions of authors expressed herein do not necessarily state or reflect those of the United States Government or any agency thereof. 


\section{DISCLAIMER}

Portions of this document may be illegible in electronic image products. Images are produced from the best available original document. 
20: DH:

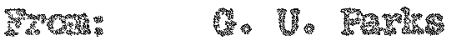

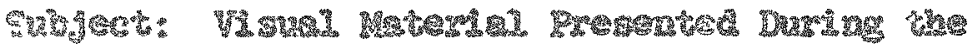

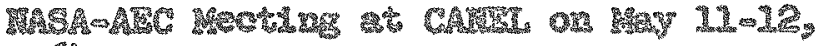
2964.

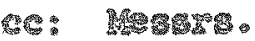

蛙. 001

R. I. Strovgh

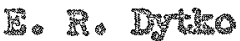

II 13 (3)

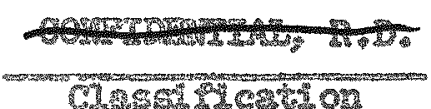

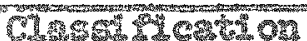

3

(P)

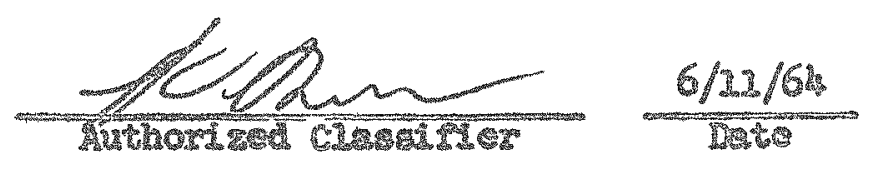

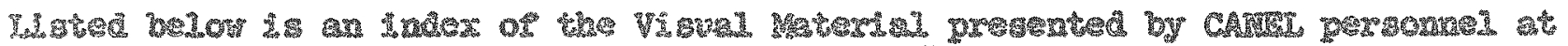
Th

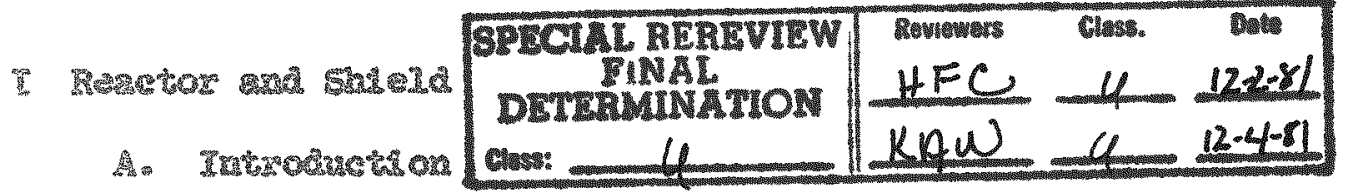

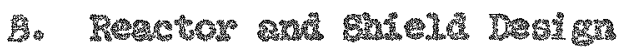

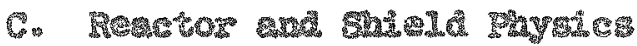

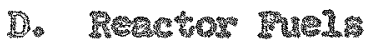

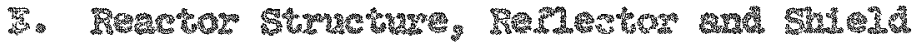

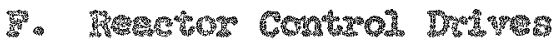

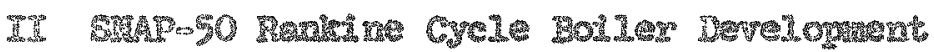

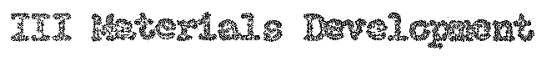

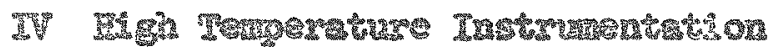

Y. Pure Derelogasent
Pag

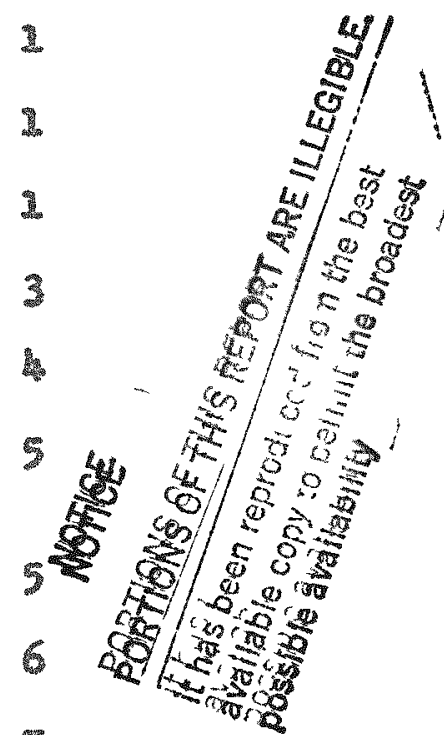

$\eta$

T
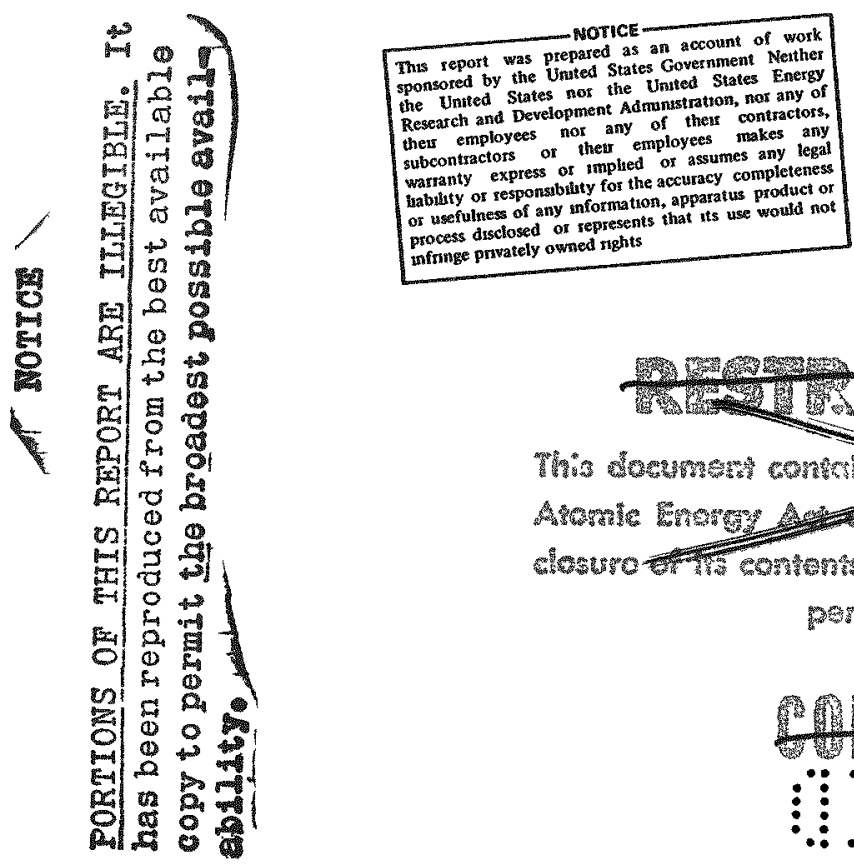

CLASSIFICATION CANCELIJED

Corto

BY AUTHORITY OF DIVISION OF CLASSIBICARTON

BY: TED REDMON DATE: $\angle 1-7-25$ geess assclosed or represtist

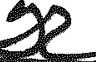

DISTREB̈UTION OF THIS DOCUMENT IS UMLATTEU,

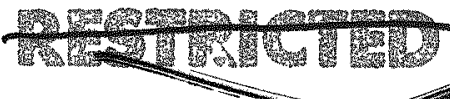

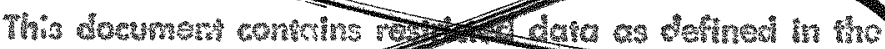
Aregrats E⿱艹

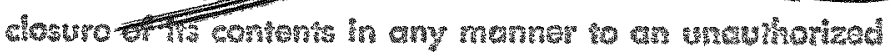
person is pohntiped.

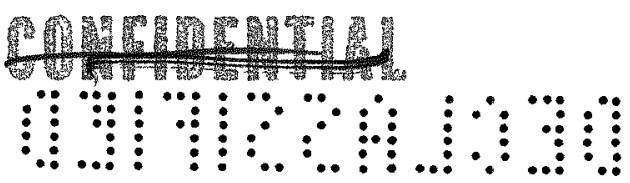




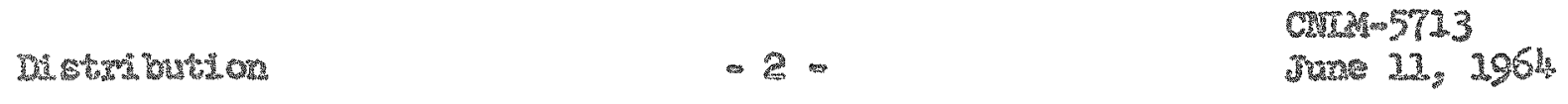

1 Remctor and she

A. Trtsoductov

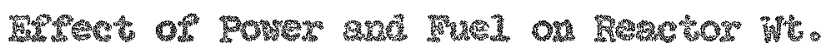

$2+9=10$

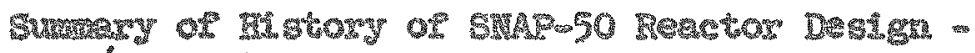
UC/ON IRE?

B. reactor and Sheld Design

General Leacription

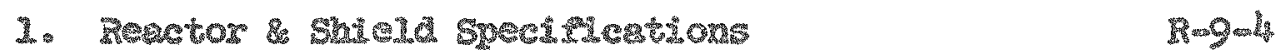

3. Reactor shield Dexign Concept Rmg-8

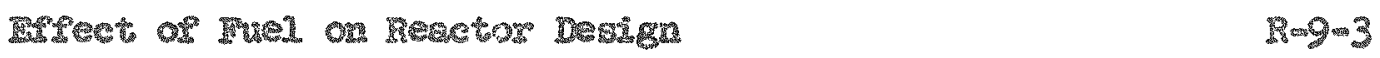

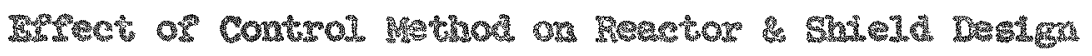

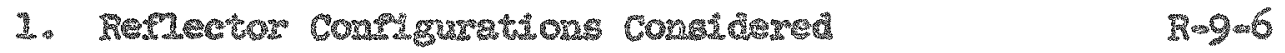

Bresent Design status

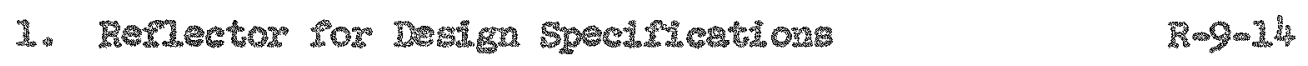

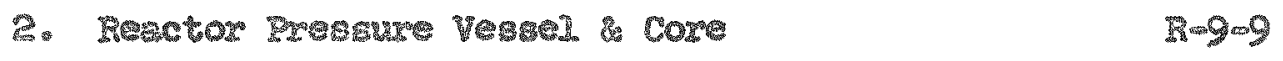

3. Wue1 Pin Design

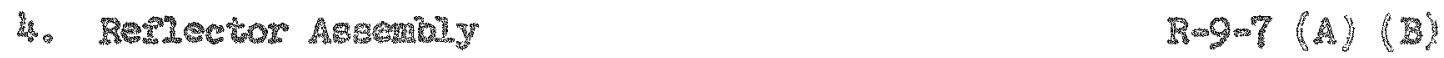

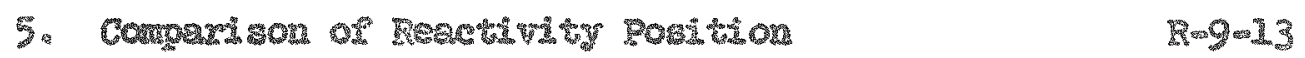

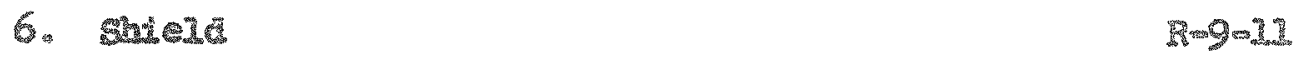

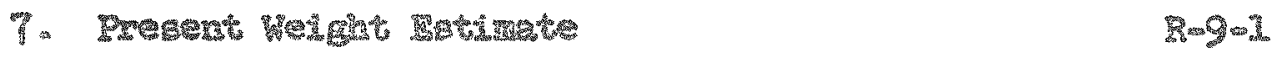

Future Dagign Prograx

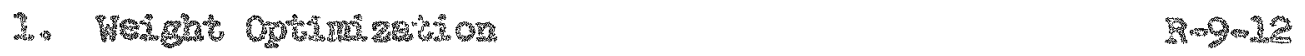

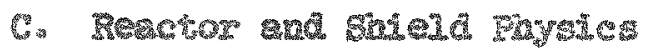

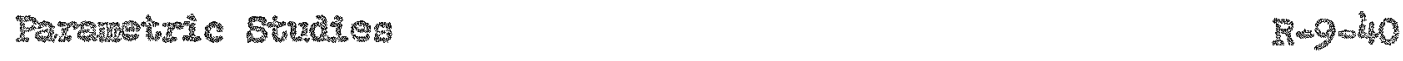

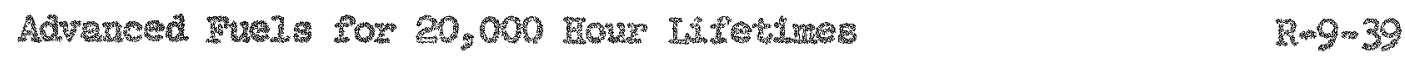

CCAs? Mux 


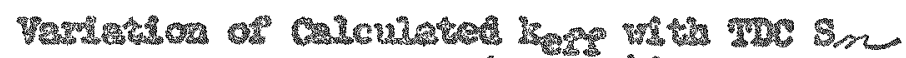

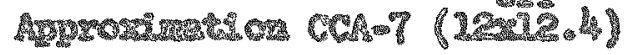

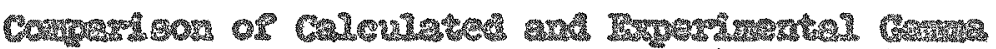

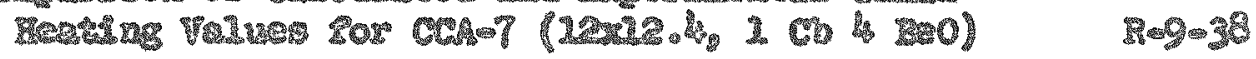

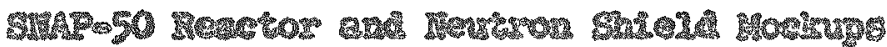

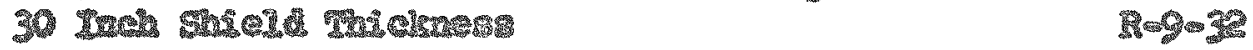

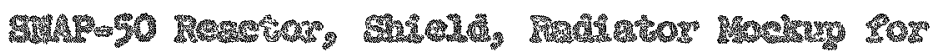

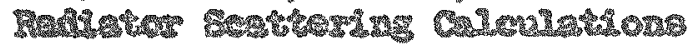

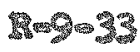

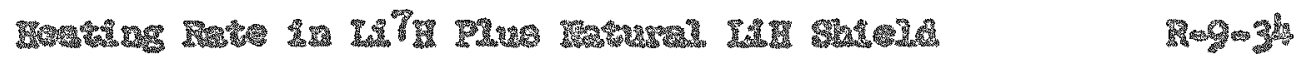

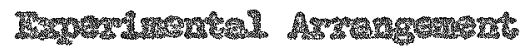

D. Rascos

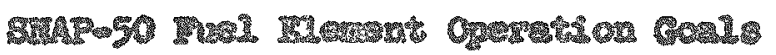
$2=958$

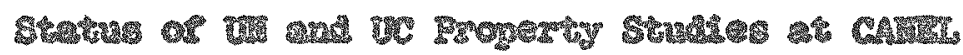
M-15 Adraresc 26. 5

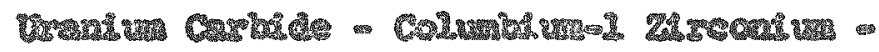
compatumaty counde

The 24

16.

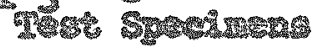

a.

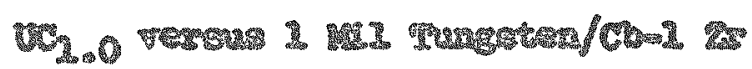

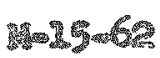

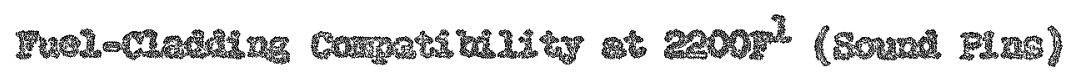

1965

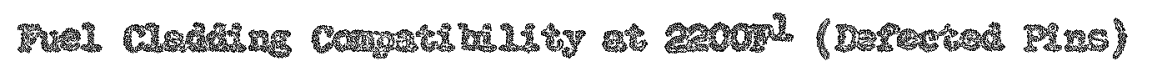

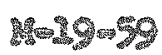

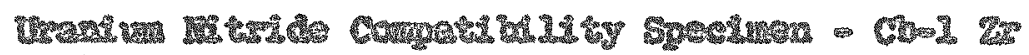
M11.5 Cas

Ny-2 2

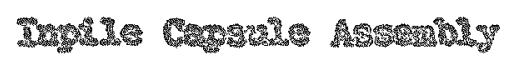

34. 396

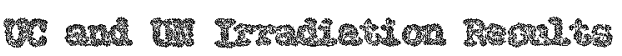

S.9.6.

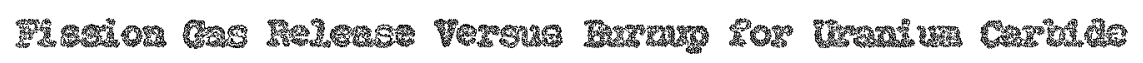

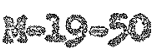

PA

19. 90

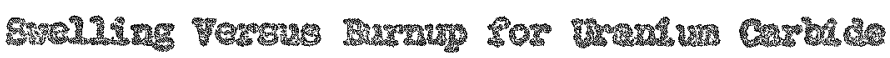

II: 29

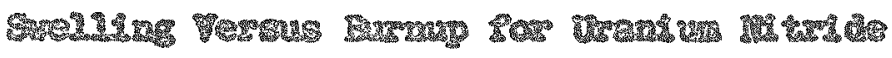

29.

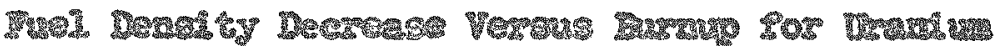
Cas?ude

som

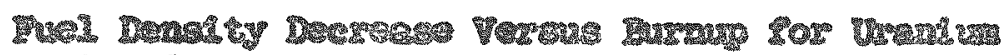

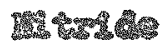




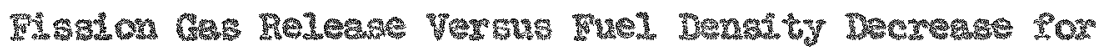
Imanu coridide

Mas

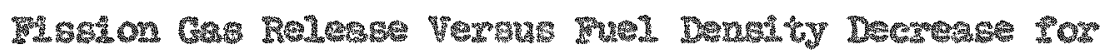

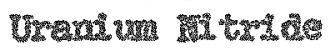

$M=2006$

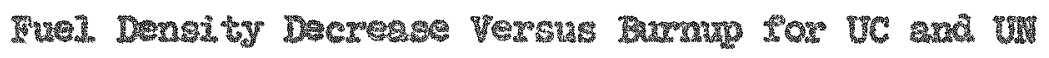
$\operatorname{Mrg} 19-51$

Fre1 Development Prograsm Goals 29.61

SWAP-50 Fuel Irrediacton Cond tron $4=29-62$

SHAP-50 UC and Un Iryeats athon Suzport Exogram M

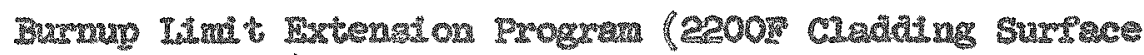
Texporstume)

$M-19-57$

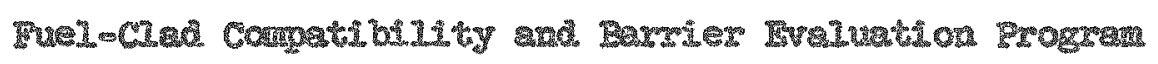

$19=19-63$

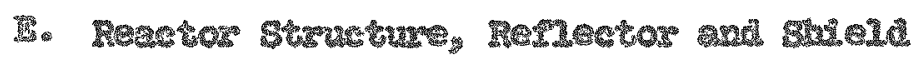

Fued chad objectures

$M=19-30$

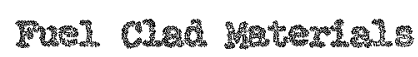

H $19-35$

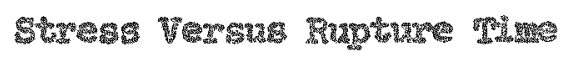

Mes 20012

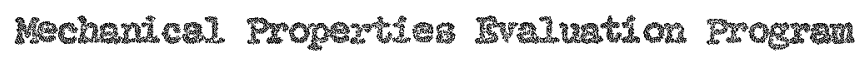

H. 19.99

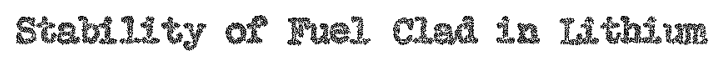

$M=1908$

stzmetural MLy objectron

M-19-31

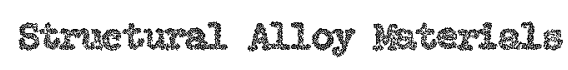

$M=19-19$

Advenced struetura Al Aloys

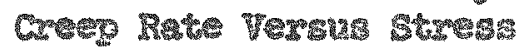

M $200-20$

Rosesure Vested Mew progran

$M=32$

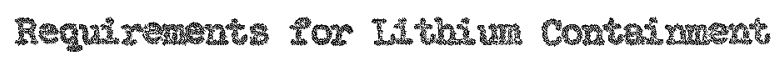

M. $19-42 \times$

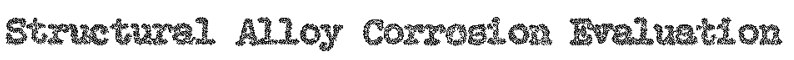

MAxto 39

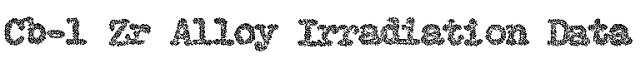

$319=20$ 


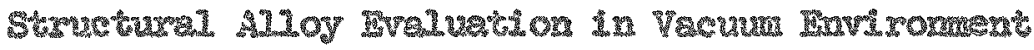

$M-19=36$

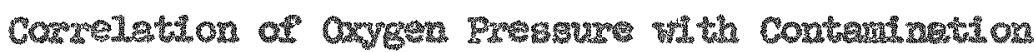
or Cber 2 $2130 y$

Mos 20

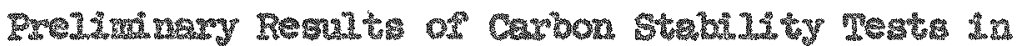
Vecuxan

$M=29-37$

Renector Semport structure

$4-20-15$

Reriector Matral

Mas $-20-14$

Weutron shield Requm ments

$M=19-33$

Hewtron Shald Matratals

$m-19-34$

Suratery of ISI Thechnology

Mos 9966

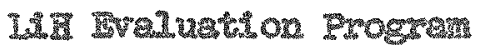

$M=19-67$

2. Resctor Control Dyduea

Co-Iireer Control Drve, Contsued

$7 \times 9=2$

Dety Cye2s

High Twaperature Control component Ixparence

Rom $\log 3$

Conkrol Dxive Corgonent Perfoxnene objectuves

$8=9-4$

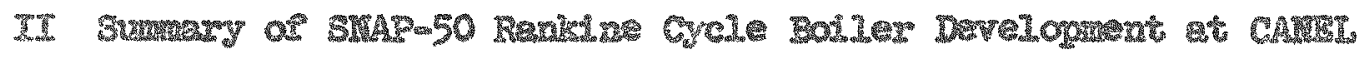

Past Hat

T)

Feat sarehanger

2.m.

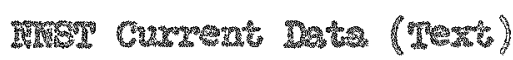

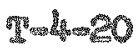

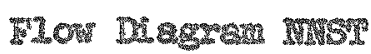

$2=6.96$

gext

tres

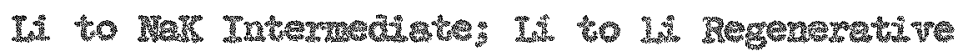
Heat Nxchomgers

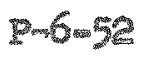

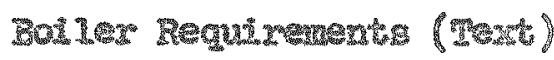

I 


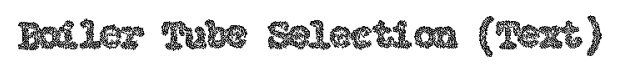

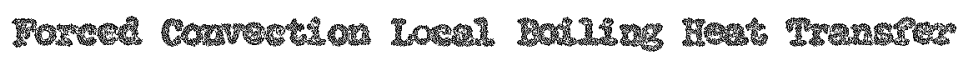
Veras Iocs Gusts

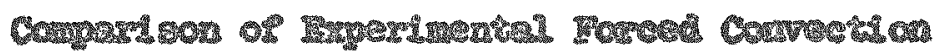

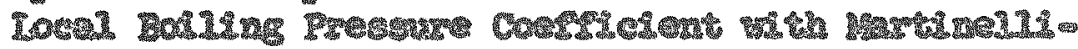

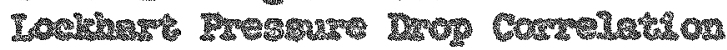

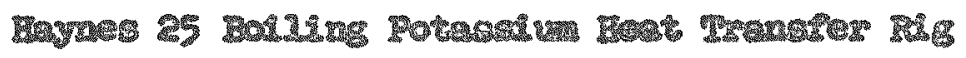

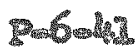

Tho Thase potras Das: (Text)

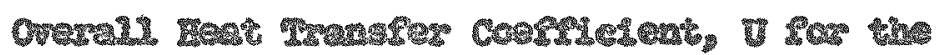

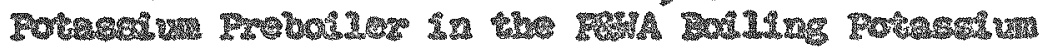

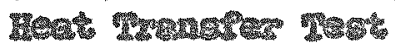

503

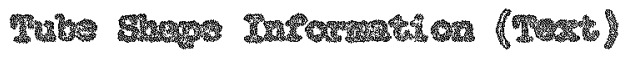

Tyes

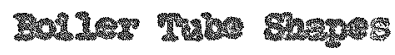

7. 2.61

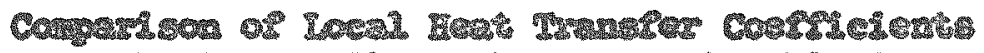

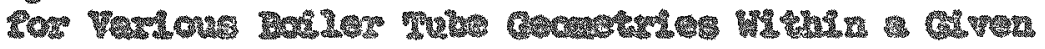

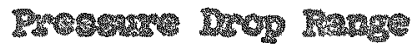

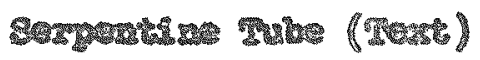

Fed 13

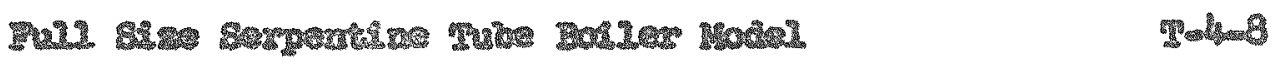

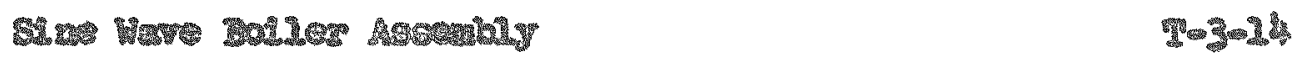

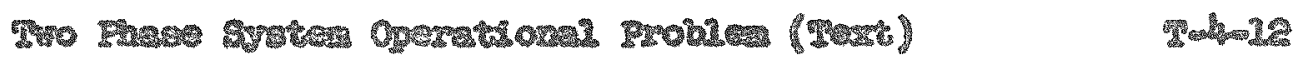

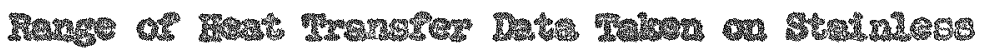

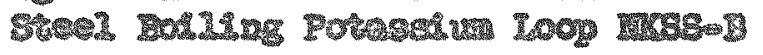

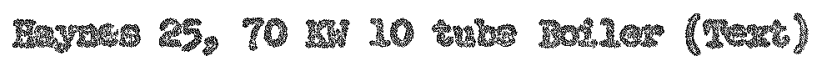

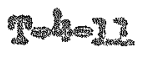

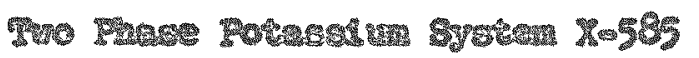

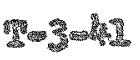

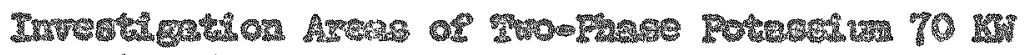
syoten Test

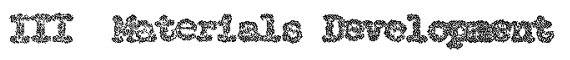




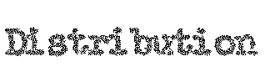

IV Kgh Temperature Tnstrumentaton

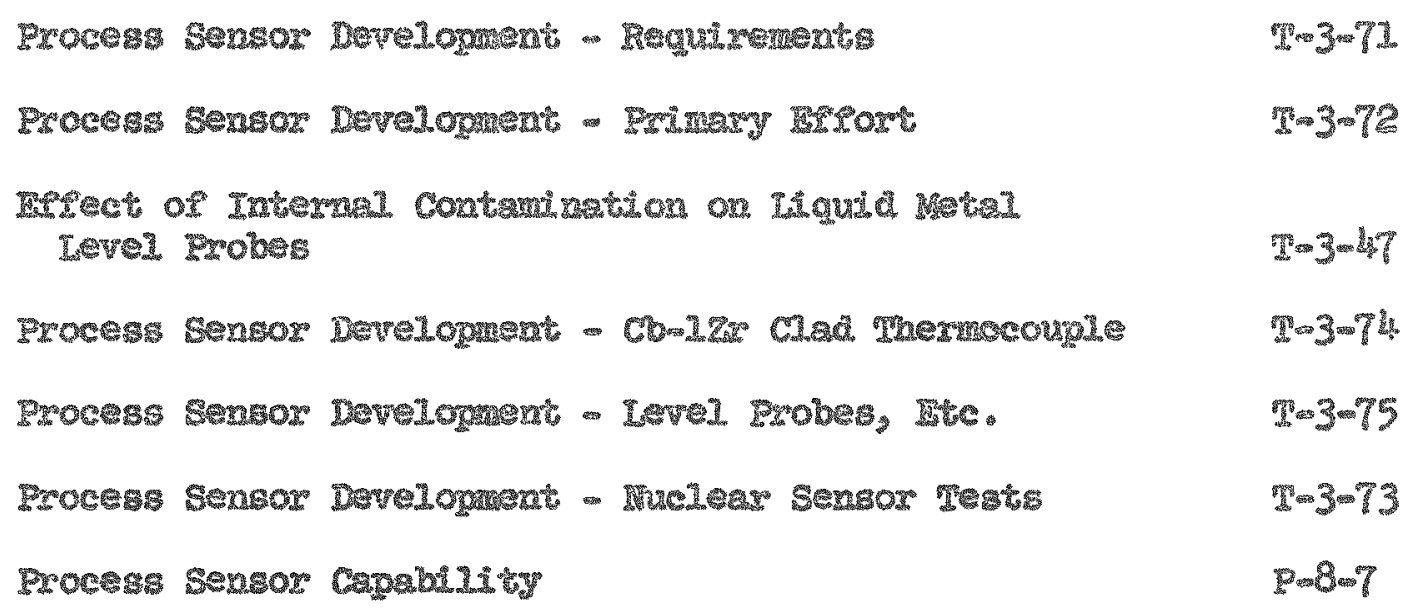

V Damp Devezognerat

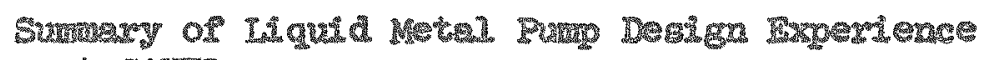
st CANES

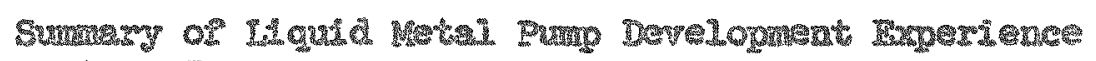
at CANEL

$p=90$

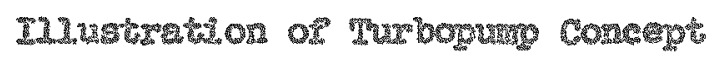

$p-5=38$

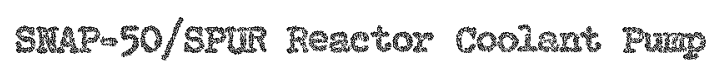

$p=8-32$

SWAP-50/SPUR condenesto Barm

P. 33

SRMP-50/5YUR Condensox Contant Furn

$\operatorname{pos}_{0} 32$

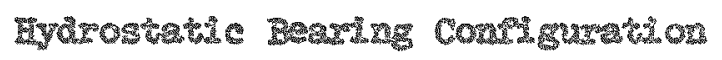

$2 \times 8 \times 9$

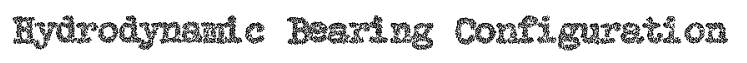

P. 8.68

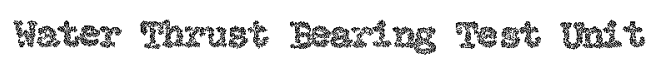

$\operatorname{Ps} 8.72$

L.M. Beareng MI:

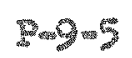

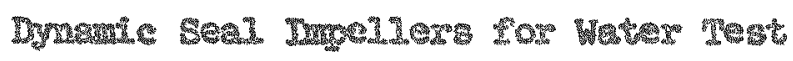

9.8.

Intersace on Dynome Seat

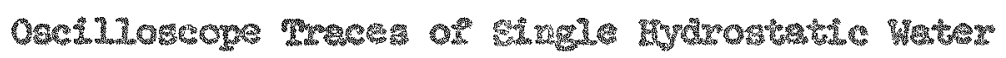

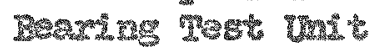




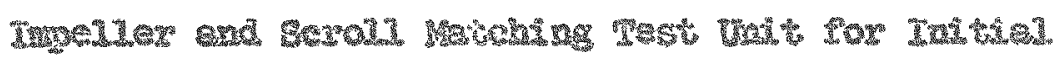
Reactor coolant Prin? 


\section{EFFECT OF POWER AND FUEL BURNUP ON REACTOR WEIGHT}

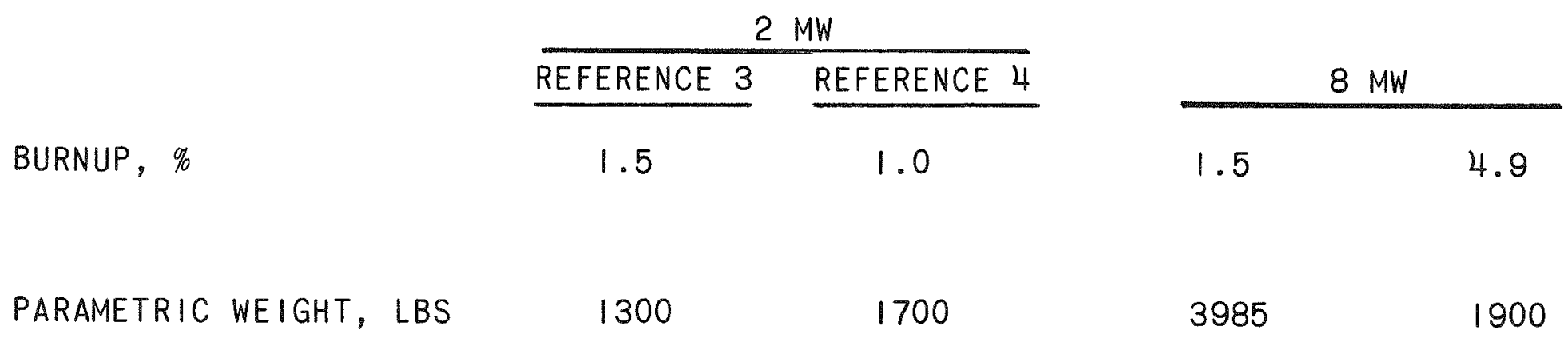

\section{:....:}

::... ......

¿.....

….... $\therefore \because$

$\because \cdots$ . ...... $\therefore \therefore$ ¿: :...: 


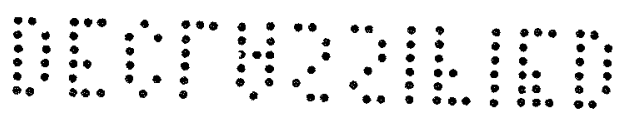

\section{SNAP-50 REACTOR AND SHIELD DESIGN SPECIFICATIONS}

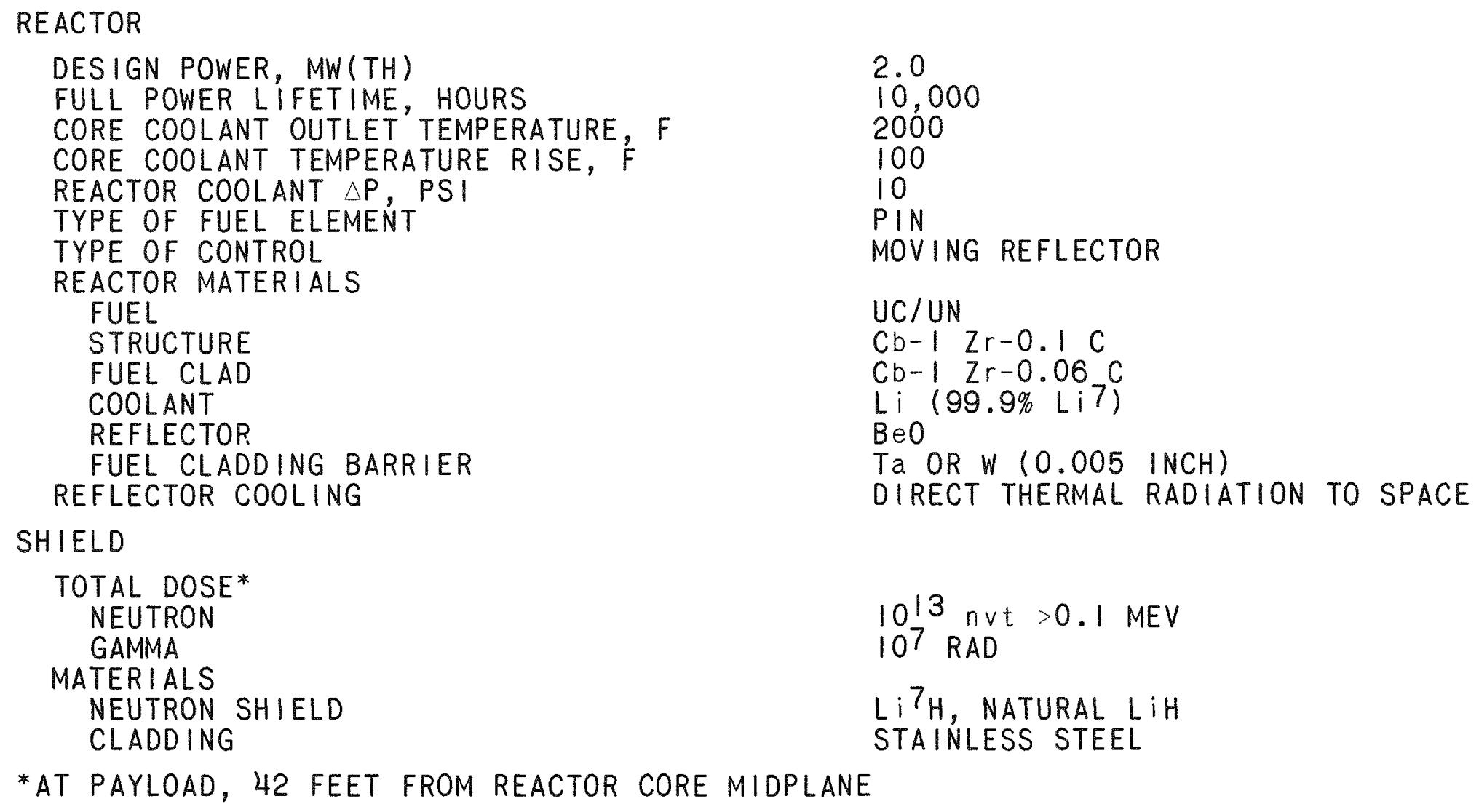

$$
2.0
$$$$
10,000
$$$$
2000
$$$$
100
$$

*at PAYLOAD, 42 FEET FROM REACTOR CORE MIDPLANE

$\therefore \cdots:$ :::..: ....... $\vdots \ldots$ (a..... $\because \because$ $\therefore \because$ $\therefore \because$ $\ldots . .$. $\therefore \therefore$ $\vdots .$. $\therefore: .:$ 
SNAP-50 REACTOR AND SHIELD DESIGN CONCEPT

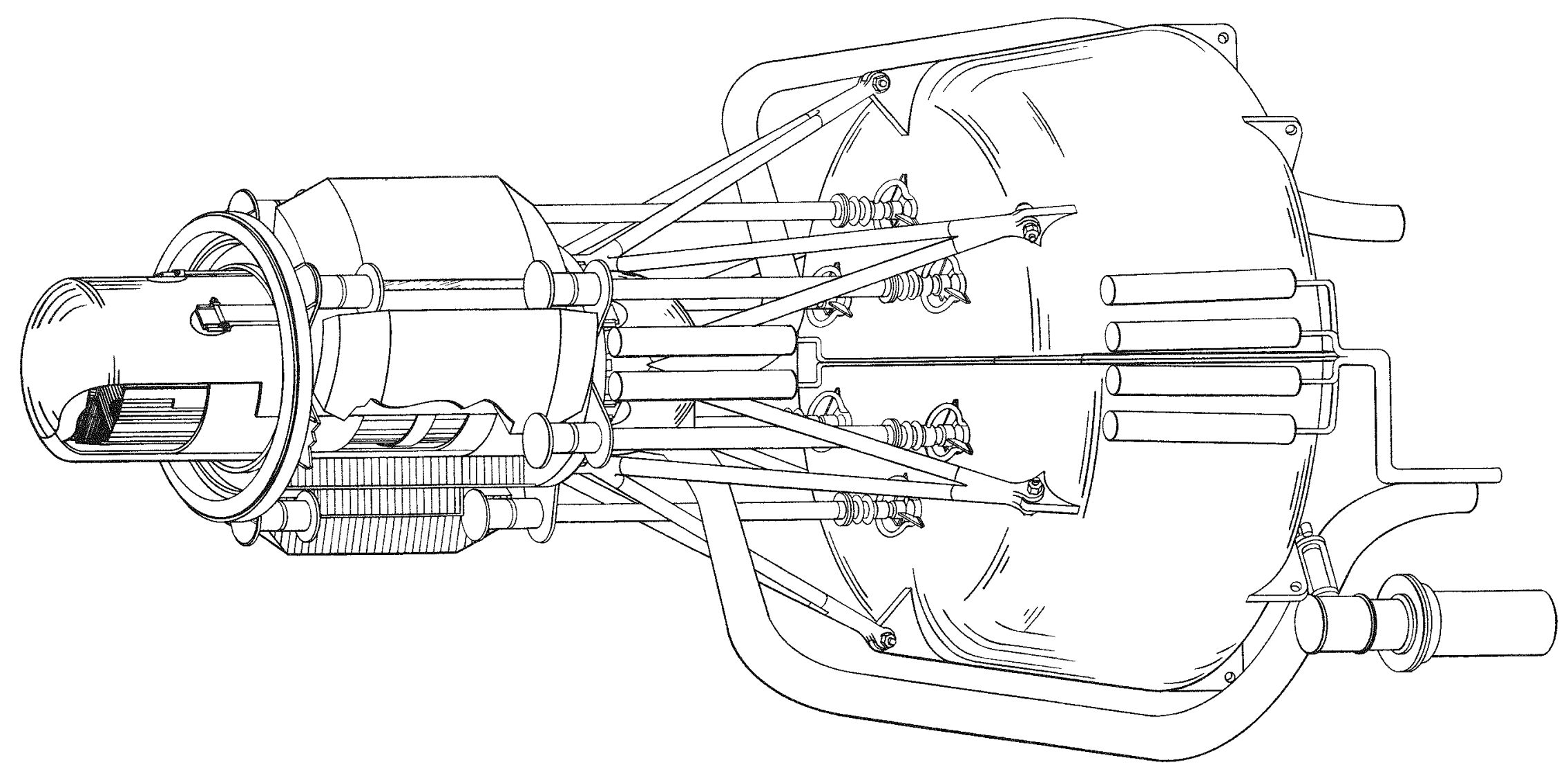




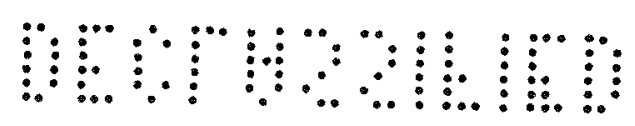

RESULTS OF REACTOR FUEL PARAMETRIC STUDIES FIRST GENERATION FUEL

PRESENT

UC/UN

PERFORMANCE ASSUMPTIONS

STUDIED

1. FUEL DENSITY,

$\%$ THEORETICAL

2. MAXIMUM FUEL

TEMPERATURE, F

3. FISSION GAS' RELEASE, \%

4. MAXIMUM BURNUP, $\%$

5. FUEL CLAD

\section{RESULTS}

6. PRESENT REACTOR DESIGN POSITION (REF. 4)

FISSION GAS RELEASE, \% 20

MAXIMUM BURNUP, $\%, 1.0$

MAXIMUM POWER DENSITY,

$\mathrm{KW} / \mathrm{CC}$

ESTIMATED ENGINEERED

0.280

WE IGHT, LBS

1800

7. BACKUP REACTOR DESIGN POSITION

MAXIMUM BURNUP, \%

MAXIMUM POWER DENSITY,

$\mathrm{KW} / \mathrm{CC}$

ESTIMATED ENGINEERED

WEIGHT, LBS

95
2300
1,10
$1.0,1.5,2.0$

$\begin{array}{ccl}95 & 95 & 95 \\ 2300 & 2500 & 2300 \\ 10 & 10 & 10 \\ \text { Cb- I Zr-0.06 } & \mathrm{C} \\ 1500 \text { PSI } & \\ 2200 \mathrm{~F} & \end{array}$

OTHER CASES

FOR COMPARISON

9OUC-ZrC UC-IW $\mathrm{UO}_{2} \quad \mathrm{UO}_{2}-\mathrm{BeO}$ SECOND GENERATION
FUEL

60UC-W $804 C-W \quad 8040_{2}^{-W}$

8. HIGHER POWER LEVEL REACTOR EXAMPLES AT $8 \mathrm{MW}$

MAXIMUM BURNUP, \%

FUEL CLAD

MAXIMUM POWER DENSITY,

$\mathrm{KW} / \mathrm{CC}$

ESTIMATED ENGINEERED

WE I GHT, LBS

$\begin{array}{llll}1.5 & 1.6 & 1.1 & 1.4 \\ 0.38 & 0.44 & 0.24 & 0.15 \\ 1500 & 1350 & 1900 & 2200\end{array}$

$\begin{array}{ccc}4.5 & 5.0 & 4.0 \\ C b-1 & z r-0.06 & c \\ 0.76 & 1.14 & 0.67 \\ 2600 & 2000 & 2500\end{array}$

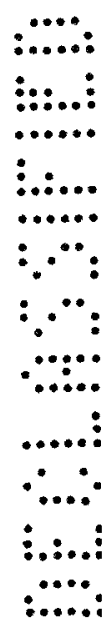

$\begin{array}{lll} & 95 & \\ 2500 & 2500 & 2700 \\ 10 & 10 & 10\end{array}$

$\mathrm{Cb}-1 \mathrm{Zr}-0.06 \mathrm{C}$ $T-222\left\{\begin{array}{l}4000 \mathrm{PSI} \\ 2200 \mathrm{~F}\end{array}\right.$ 
मओआया।

\section{REFLECTOR CONFIGURATIONS CONSIDERED FOR SNAP-50 REACTOR}

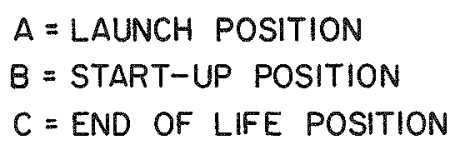

TRANSLATING

VENETIAN BLIND

ROTARY

(SNAP-8 TYPE)

PETAL
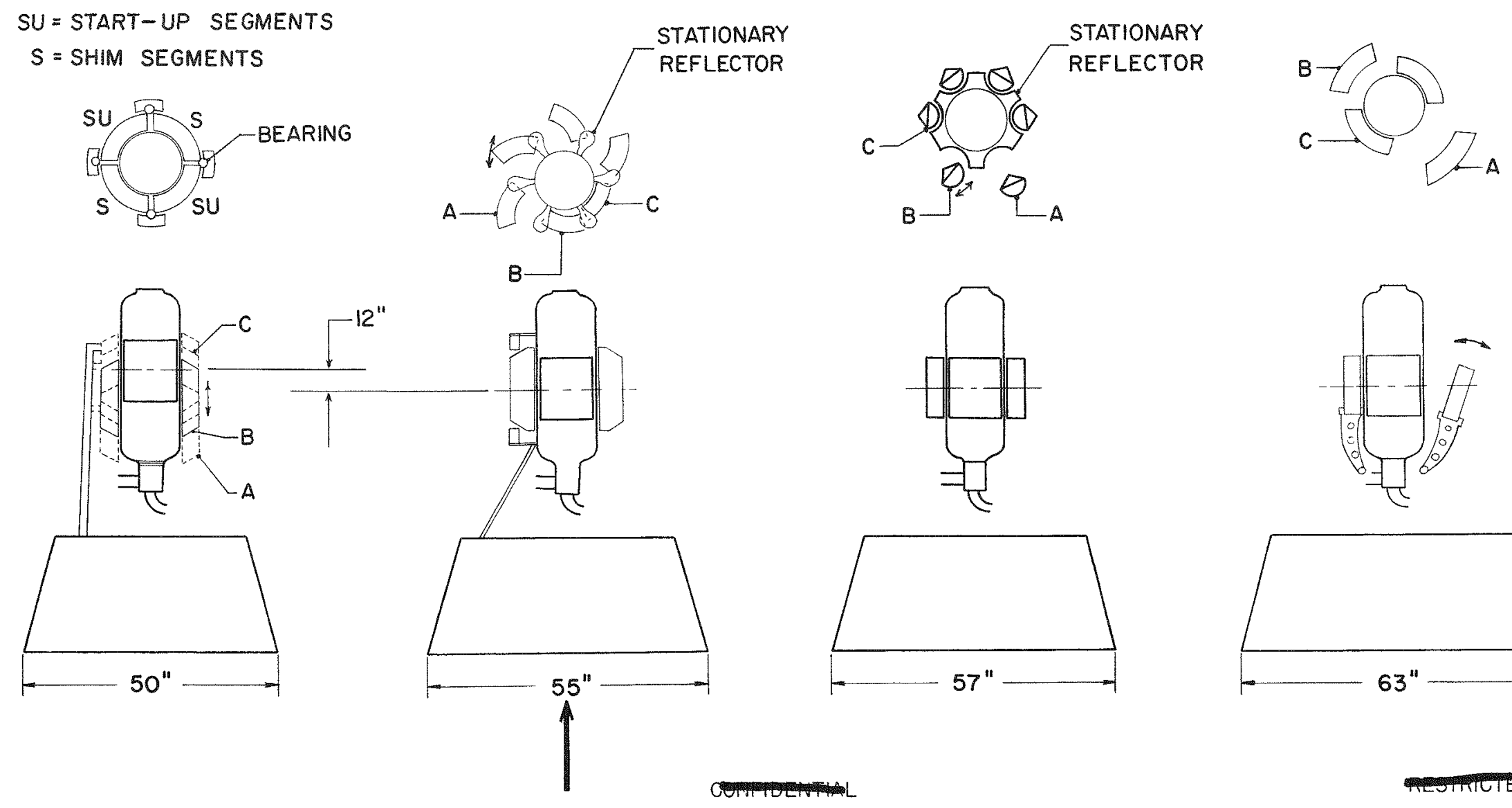

$\therefore: \ldots:$

$\therefore$

(.....

$\vdots . . .$.

$\because$

$\because$

$\because \because$

$\because \because:$

(......

$\therefore$

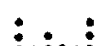

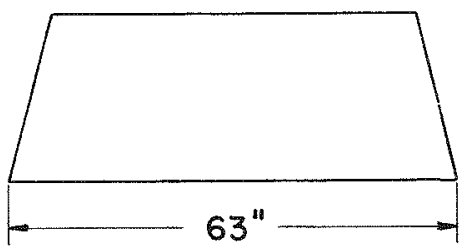

:....:

:....: 


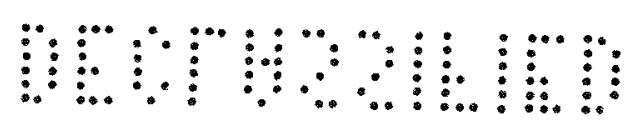

\section{REFERENCE REACTOR 4 DESIGN SPECIFICATIONS}

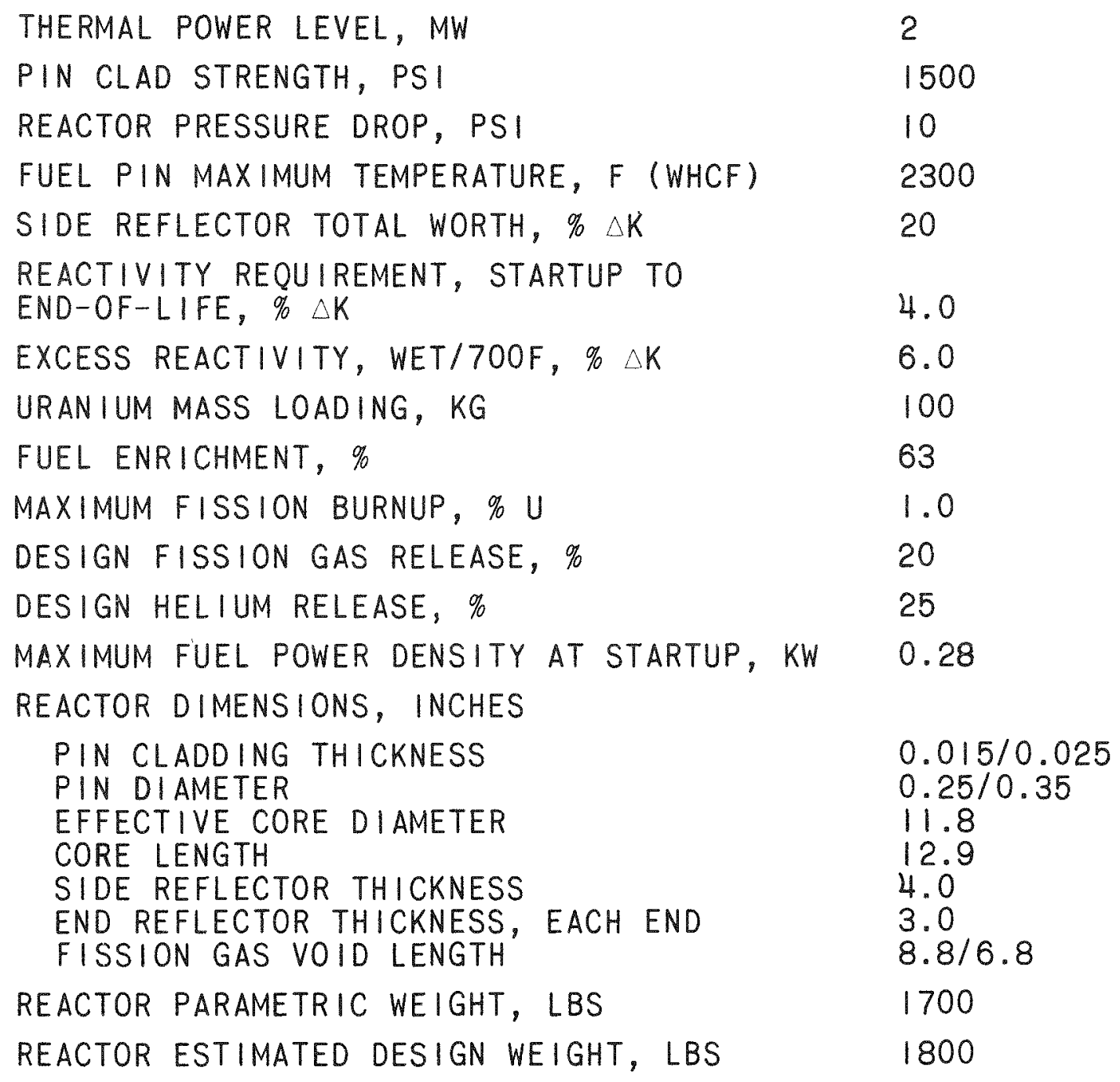
REACTOR ESTIMATED DESIGN WEIGHT, LBS

$\therefore: \cdots:$

$:: . .:$

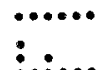
$\therefore$ $\because \because$ $\because \because$ $\because \because$ (...... $\therefore$ :.... :...:

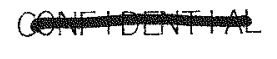




\section{REACTOR PRESSURE VESSEL AND CORE}

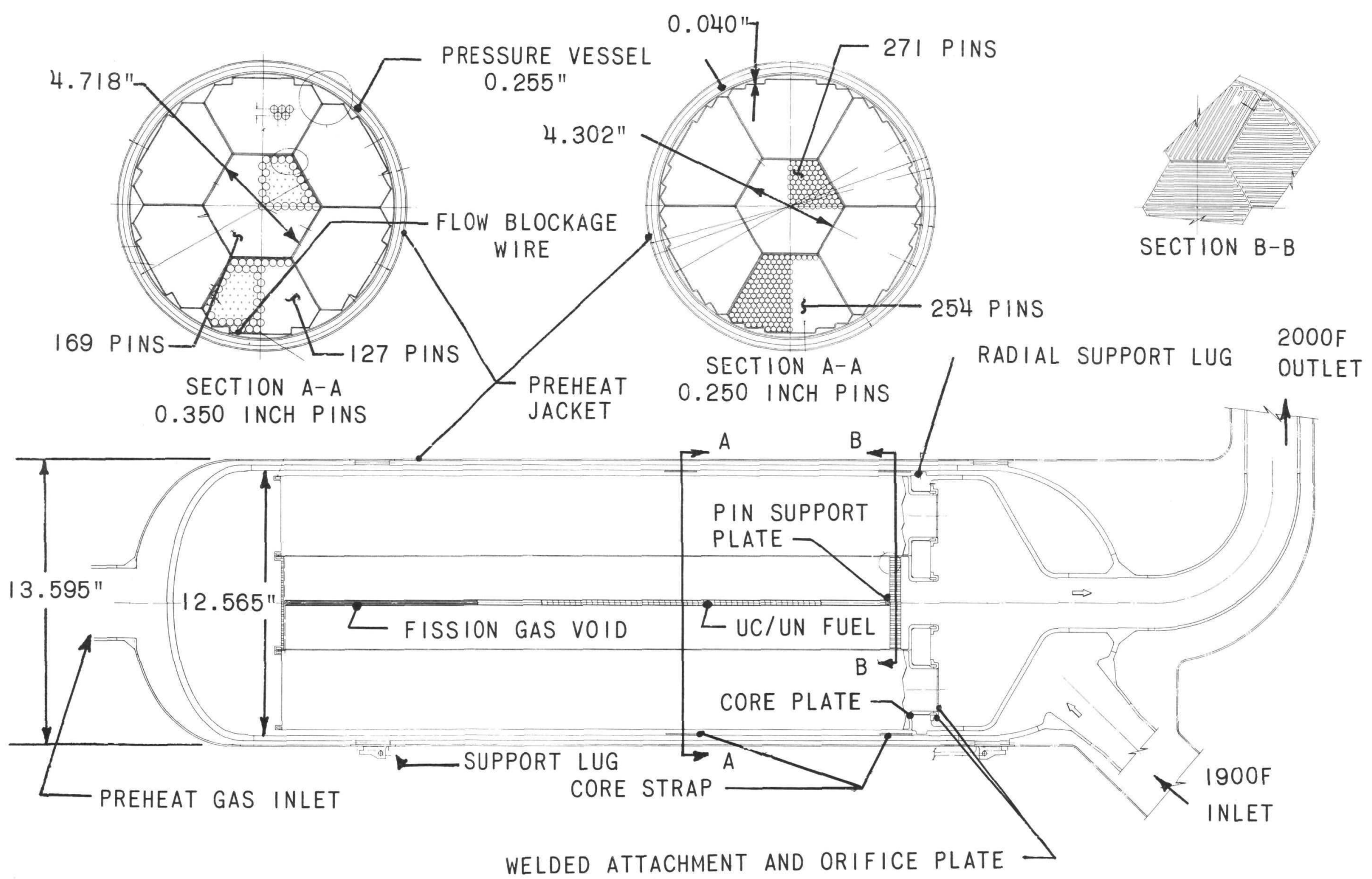

$\therefore: \cdots:$

$\vdots:: . .:$ ......

:......

$\therefore$

$\because$

$\because:$

.....:

$\therefore \therefore$

$\vdots: . .:$

$\therefore:$

WELDED ATTACHMENT AND ORIFICE PLATE 
\begin{tabular}{l}
\hdashline \\
\hdashline
\end{tabular}

REACTOR FUEL PIN - 0.250 INCH DIAMETER

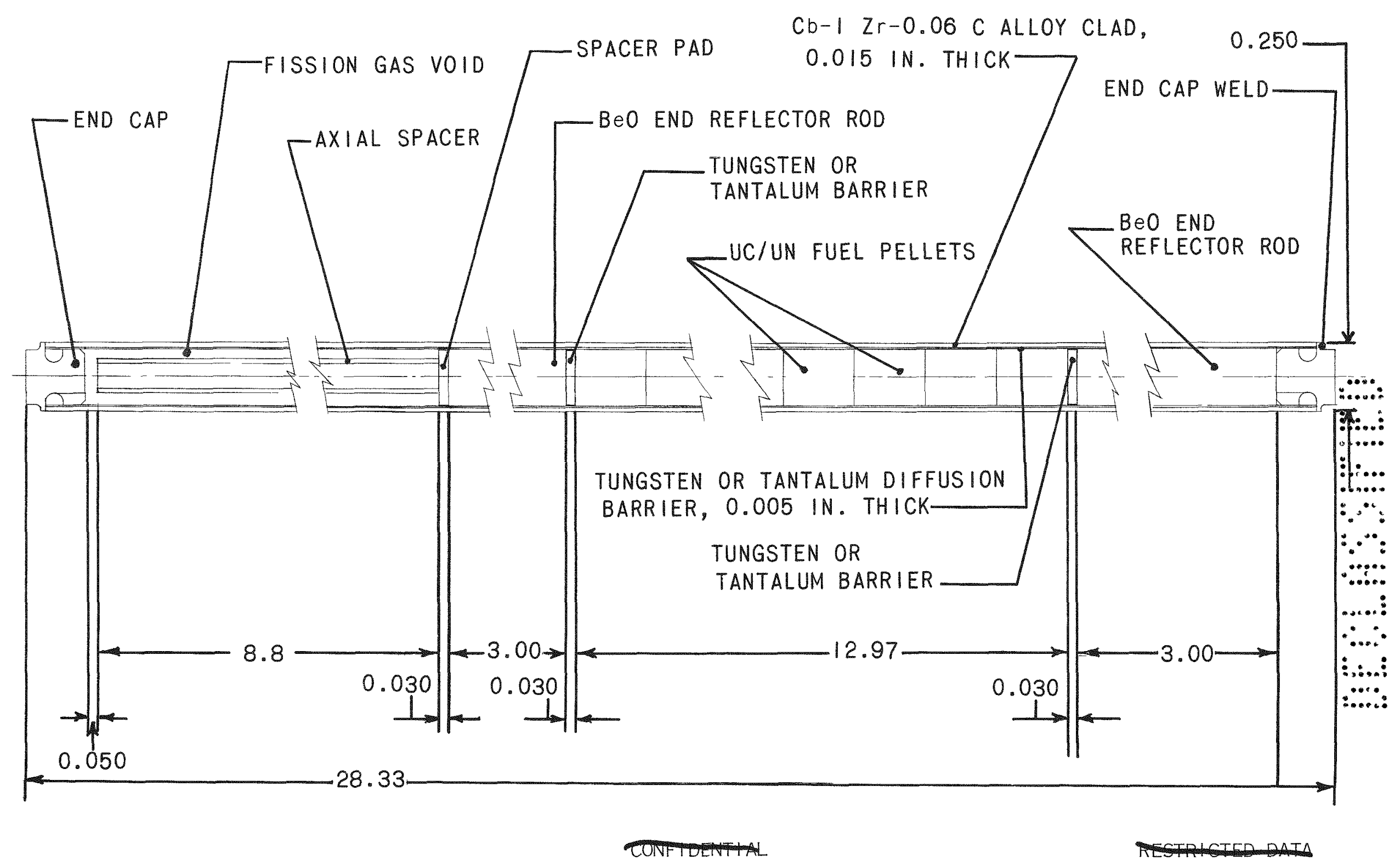




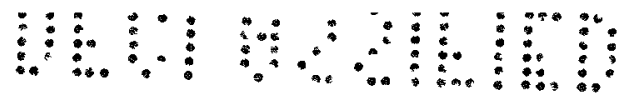

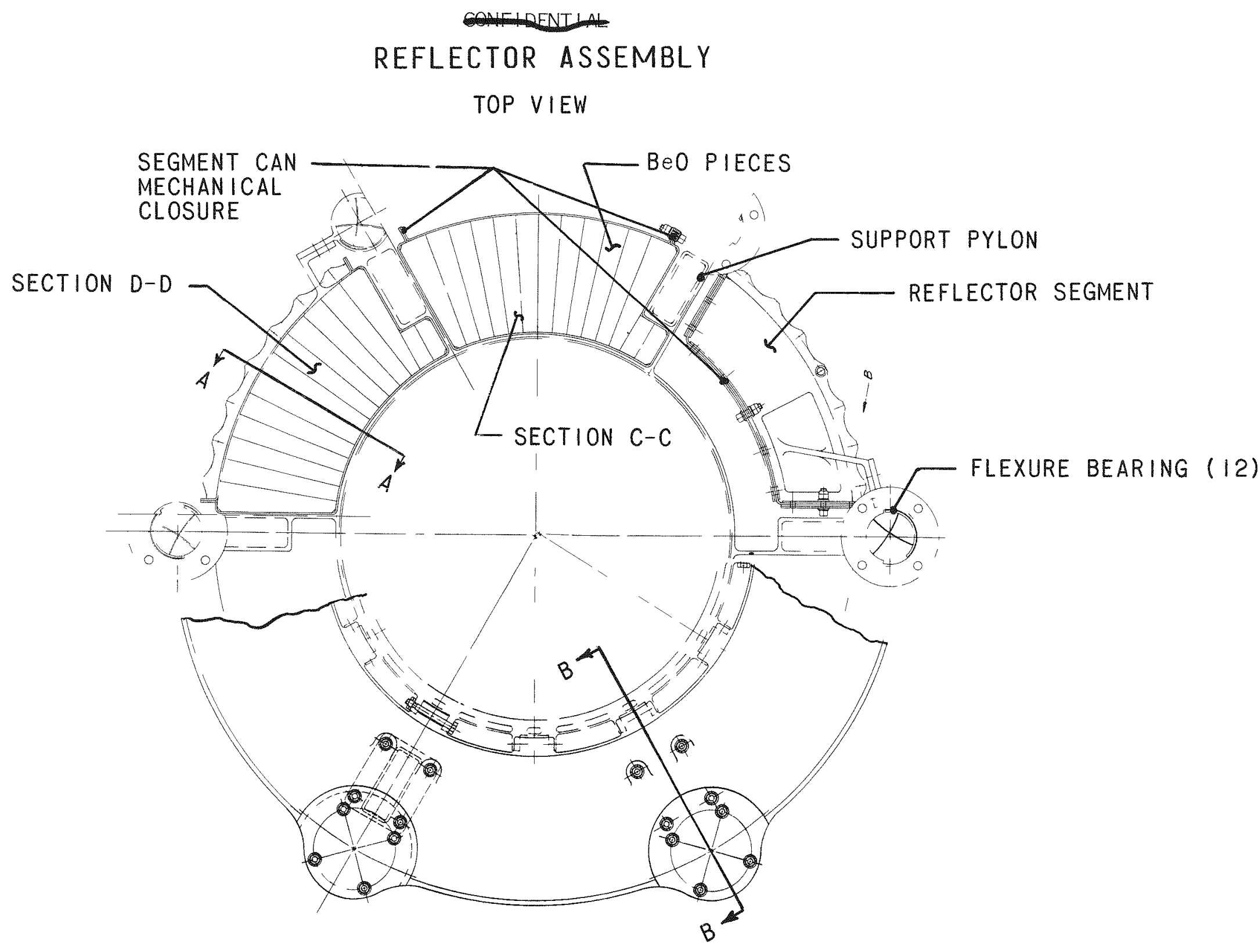

$\therefore: \cdots:$

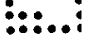

(n)

- lo...

$\therefore \because$

$\because \because$

$\because \because$

$+\cdots$

$\therefore \therefore$

¿...

$\therefore:$. 
⿶凵:

OAE IDENT LAL

REFLECTOR ASSEMBLY

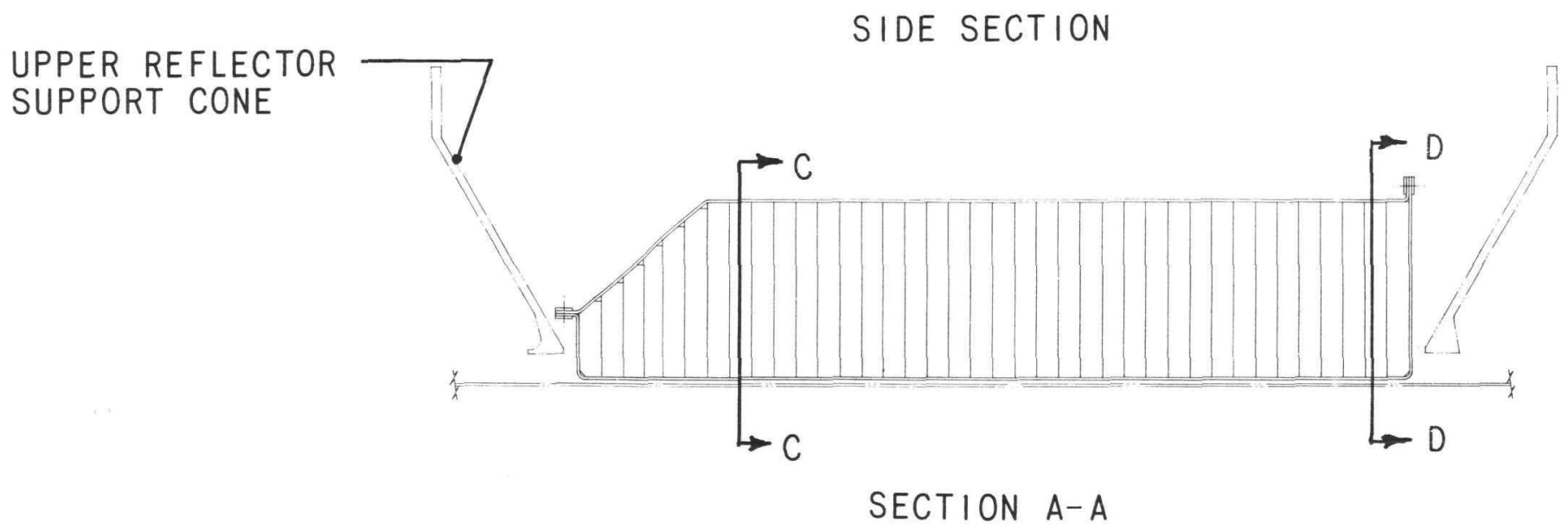

SECTION B-B

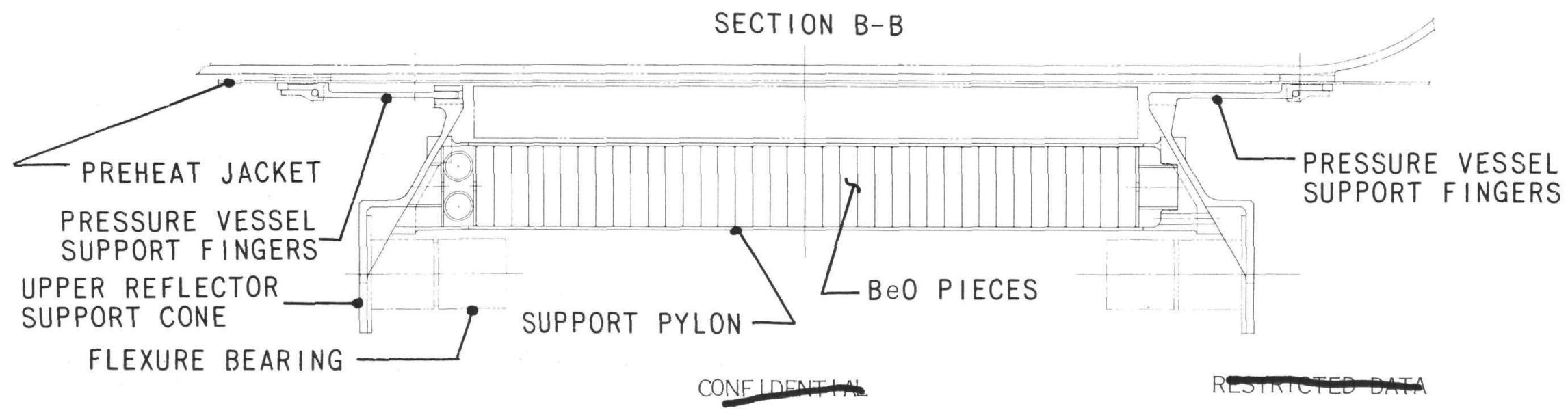

$\therefore \ldots:$

....:

$:: . .$.

$\therefore$

(......

$\therefore \because$

$\therefore$.

$\because \because$ 
आ०ण

\section{COMPARISON OF SNAP-50 REACTIVITY POSITION}

WITH OTHER SNAP REACTORS

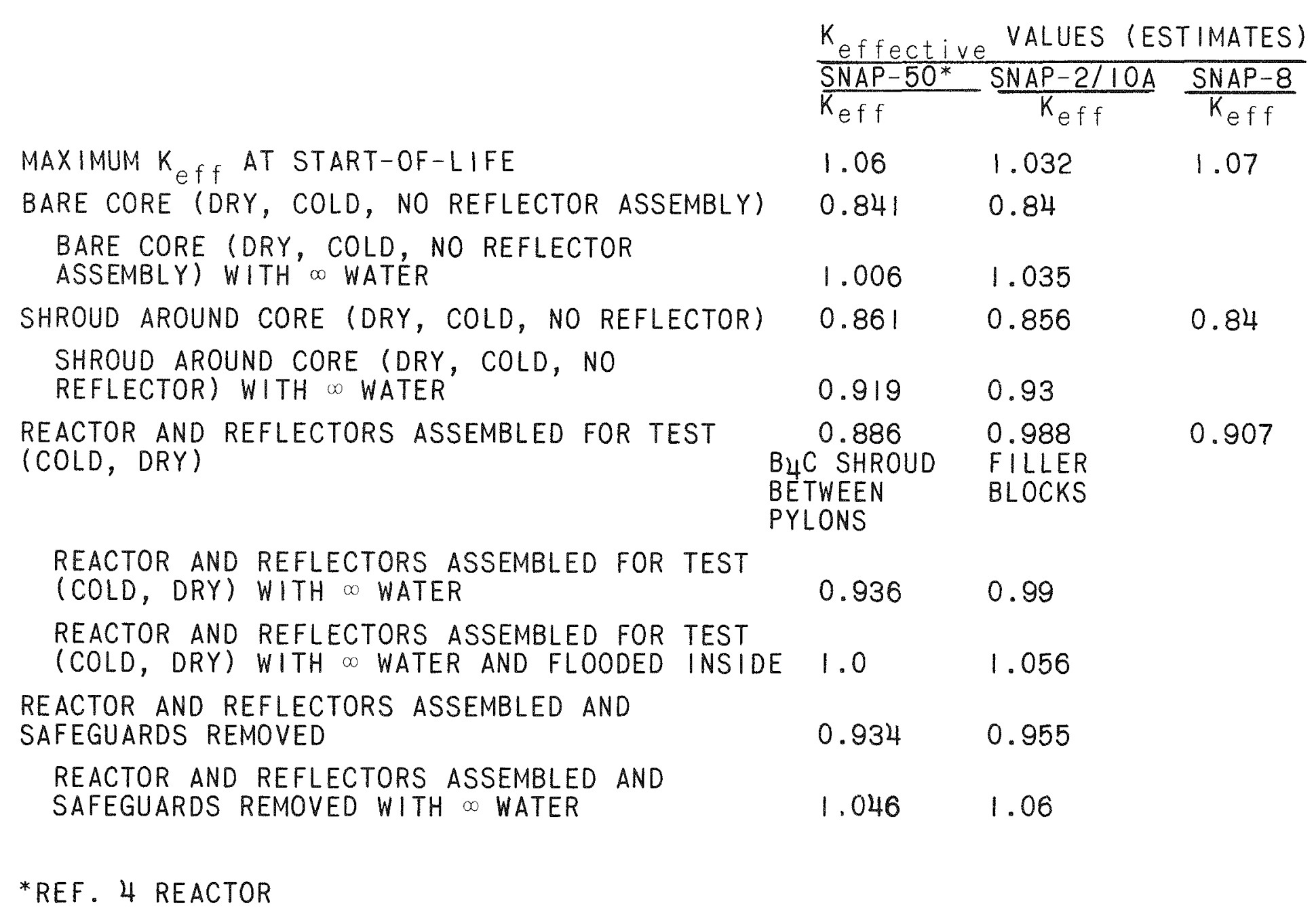


年:

\section{PRESENT SHIELD CONCEPT}

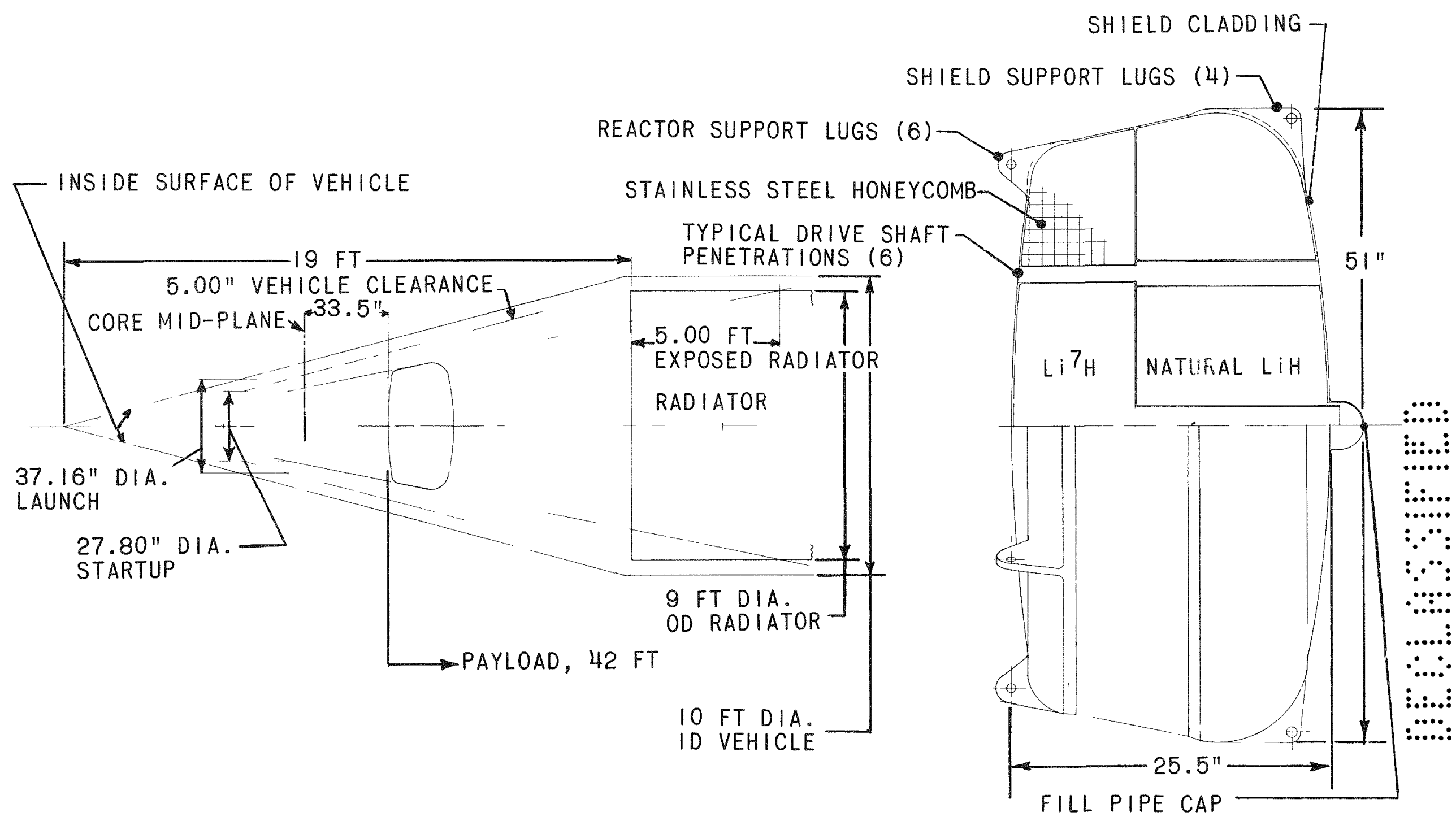




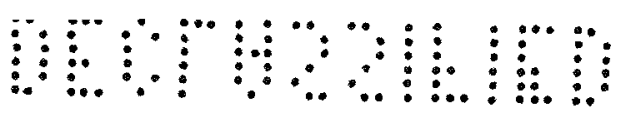

\section{PRESENT WEIGHT ESTIMATE - \\ SNAP-50 REACTOR-SHIELD ASSEMBLY}

\section{REACTOR}

PRESSURE VESSEL AND CORE

1060

SIDE REFLECTOR

460

CONTROL DRIVES, GEARING, SHAFT

270

LIQUID METAL

40

TOTAL

1830

SHIELD

LIH MAIN SHIELD

1250

LIH PIPING PATCH SHIELD

100

STRUCTURE AND CAN

490

TOTAL

1840

REACTOR SUPPORT STRUCTURE

160

MISCELLANEOUS COMPONENTS (INSTRUMENTATION, WIRING, SAFETY DEVICES, INSULATION)

100

PIPING ( INCLUDING Li)

TOTAL REACTOR-SHIELD ASSEMBLY

4010

\section{$\because \cdots:$}

:::..:

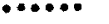

$\vdots \ldots$

(n.....

$\because \because:$

$\because \because$

$\because \ldots$

$\ldots \ldots$ :

$\therefore \ldots$

$\vdots \ldots$

a...: 


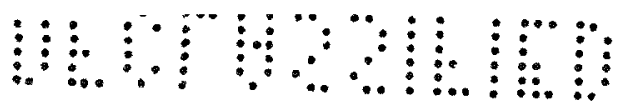

204 TOETIAL

\section{PARAMETRIC STUDIES}

FIRST GENERATION FUELS (PART 1 )

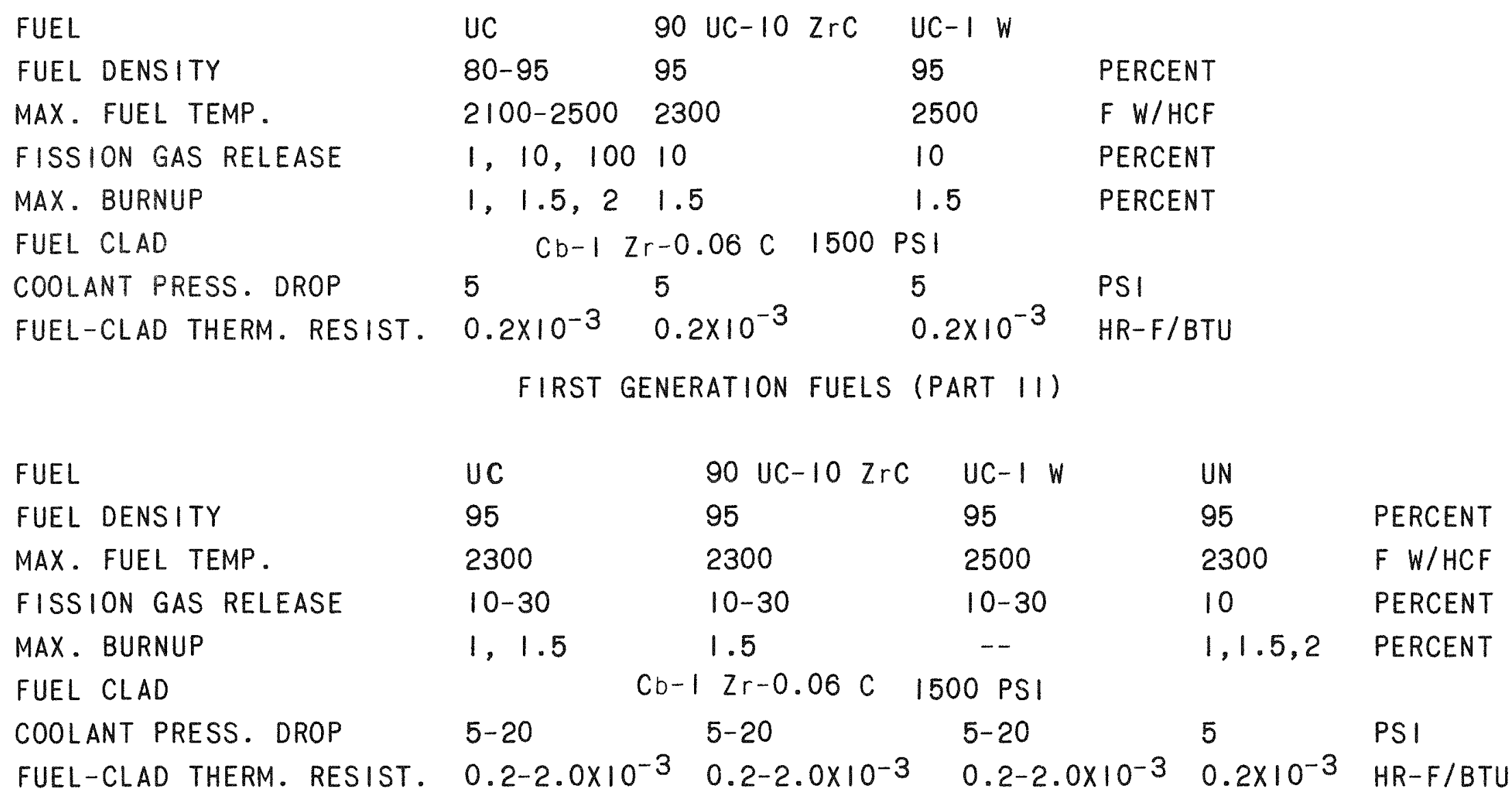

\section{$\therefore: \cdots:$}

$:: \ldots$

(n)

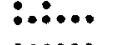

$\therefore$

$\therefore \because$

$\therefore:$

.....:

$\ldots$

$\therefore$. 


\section{ADVANCED FUELS FOR 20.000 HOUR LIFETIMES}

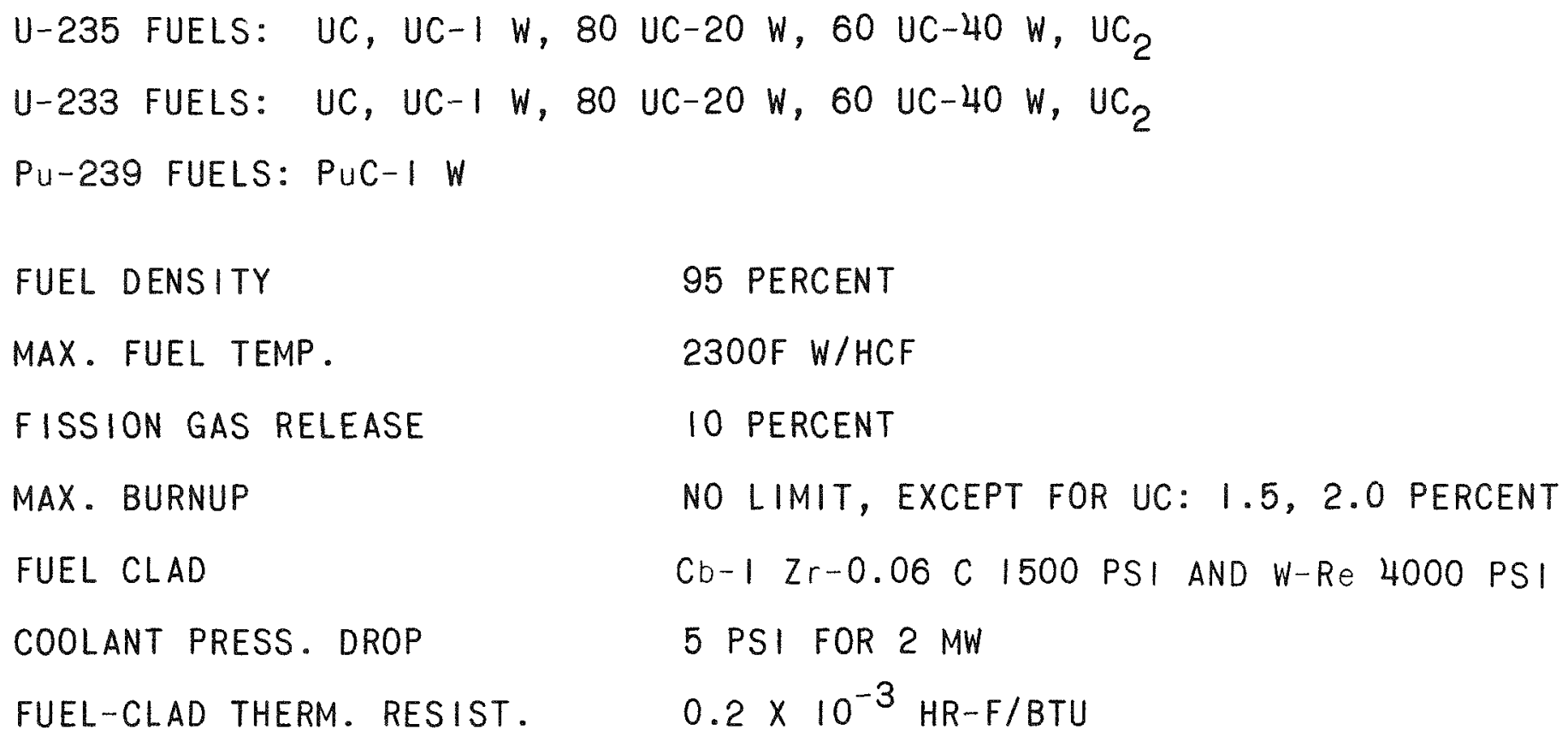

$0.2 \times 10^{-3} \mathrm{HR}-\mathrm{F} / \mathrm{BTU}$

FUEL-CLAD THERM. RESIST.

$\therefore: \cdots:$

::..:

-......

$\vdots . .$.

$\cdots \cdots$

$\because \because$

$\because \because$

$\cdots$ 


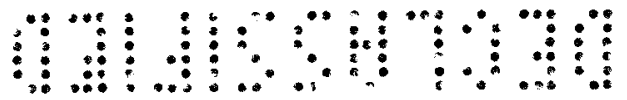

COASATAL

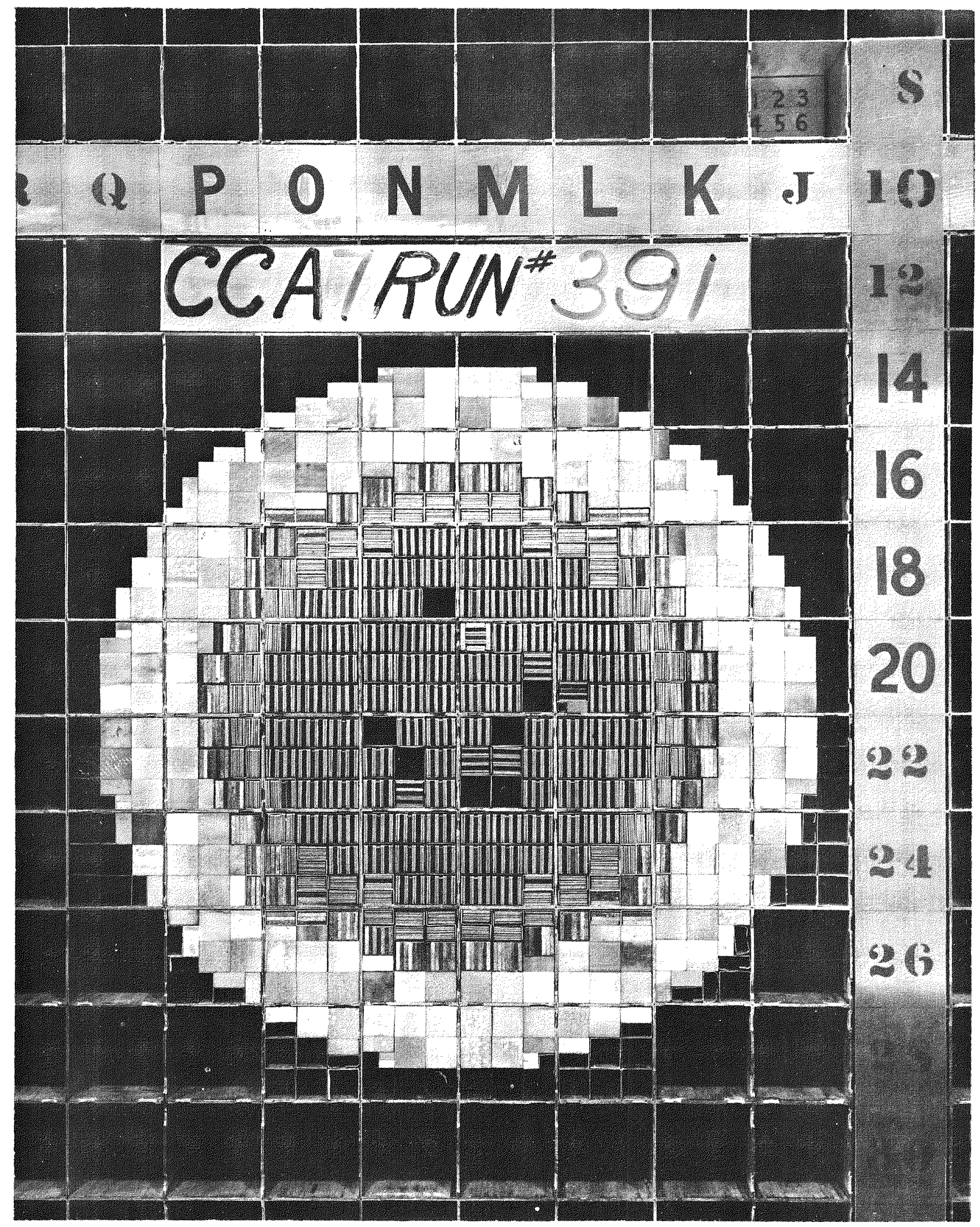

$\therefore \ldots$ 


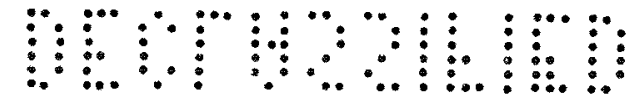

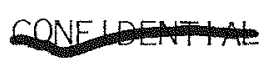

\section{VARIATION OF CALCULATED $k_{\text {eff }}$ WITH TDC $S_{n}$ APPROXIMATION CCA-7 $(12 \times 12.4)$}

$\begin{array}{lcc}\frac{S n}{S 2} & \frac{\text { FORWARD }}{1.0282} & \frac{\text { ADJOINT }}{1.0087} \\ S 4 & 1.0032 & 0.9931 \\ S 6 & 0.9992 & 0.9939 \\ S 8 & 0.9976 & 0.9929 \\ \text { 10X 1O MESH INTERVALS. LAMS-2543 } & 16-\text { GROUP } \\ \text { CROSS SECTIONS WITH CORRECTIONS TO Be }(n, 2 n) \\ \text { AND CD (FXM-6606) }\end{array}$


by

COMPARISON OF CALCULATED AND EXPERIMENTAL GAMMA HEATING VALUES FOR

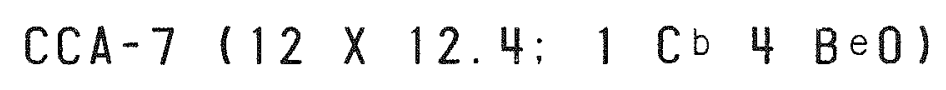

\begin{tabular}{|c|c|c|c|c|}
\hline $\begin{array}{l}\text { RAD IUS } \\
\text { (CM) }\end{array}$ & & 0.86 DISTAN & $\begin{array}{l}\text { CE FROM MIDPL } \\
6.75\end{array}$ & $\begin{array}{l}\text { ANE }(\mathrm{CM}) \\
16.9\end{array}$ \\
\hline 18.7 & $\begin{array}{l}\text { CALC. } \\
\text { EXP. }\end{array}$ & $\begin{array}{l}2.3 \times 10^{-7} \\
2.4\end{array}$ & $\begin{array}{l}2.1 \times 10^{-7} \\
2.2\end{array}$ & $\begin{array}{l}0.9 \times 10^{-7} \\
1.02\end{array}$ \\
\hline 21.6 & $\begin{array}{l}\text { CALC. } \\
\text { EXP. }\end{array}$ & $\begin{array}{l}1.4 \times 10^{-7} \\
1.5\end{array}$ & & \\
\hline 24.1 & $\begin{array}{l}\text { CALC. } \\
\text { EXP. }\end{array}$ & $\begin{array}{l}0.95 \times 10^{-7} \\
0.83\end{array}$ & $\begin{array}{l}0.81 \times 10^{-7} \\
0.80\end{array}$ & $\begin{array}{l}0.44 \times 10^{-7} \\
0.39\end{array}$ \\
\hline 26.7 & $\begin{array}{l}\text { CALC. } \\
\text { EXP. }\end{array}$ & $\begin{array}{l}0.59 \times 10^{-7} \\
0.54\end{array}$ & & \\
\hline
\end{tabular}


SNAP-50 REACTOR AND NEUTRON SHIELD MOCKUPS 30 INCH SHIELD THICKNESS

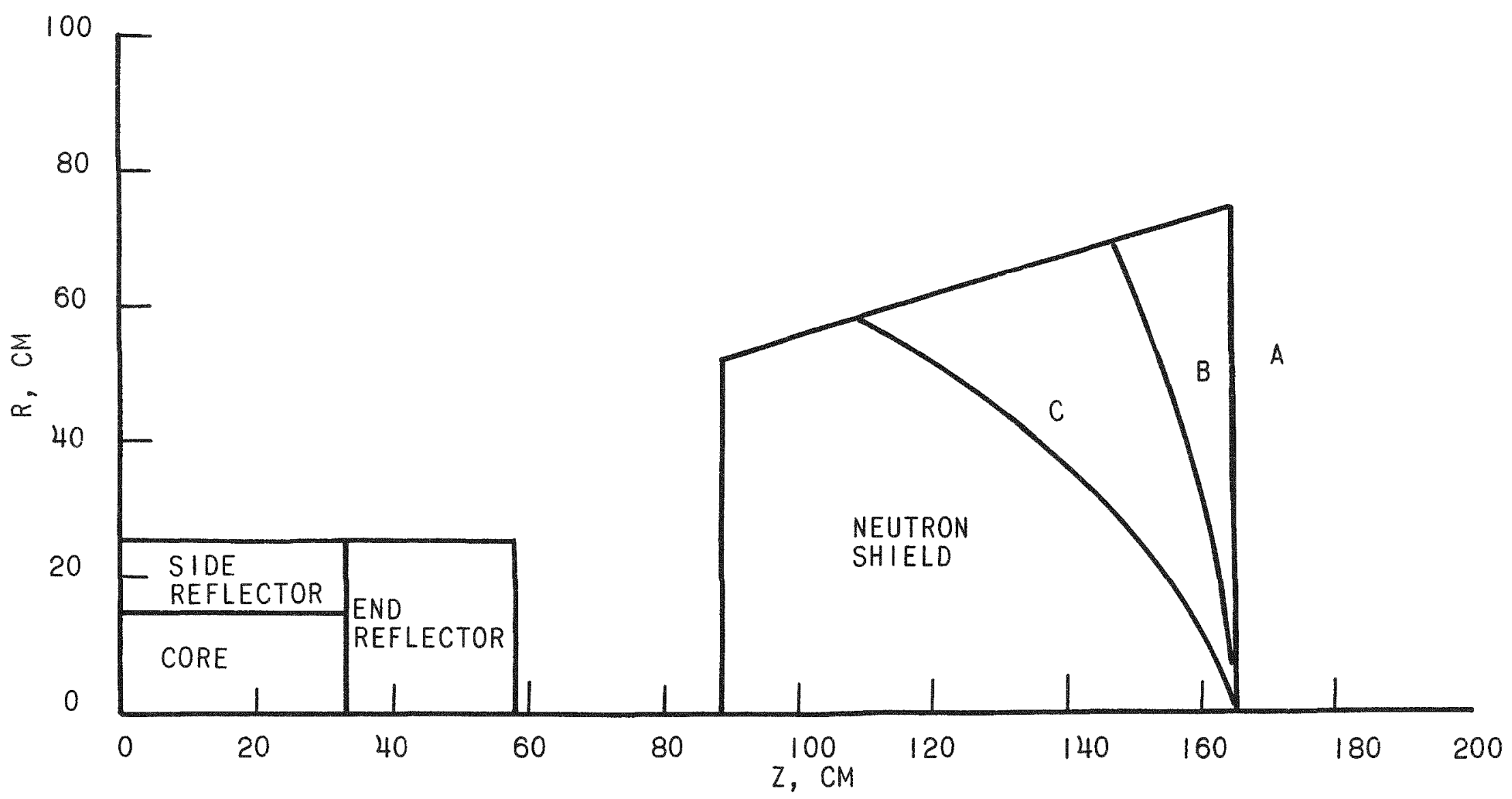

$:$

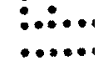

$\because \because$

$\therefore$

$\because \because$

....

$\therefore . .$.

(.....

$\therefore: .:$ 
SNAP-50 REACTOR. SHIELD. RADIATOR MOCKUP FOR RADIATOR SCATTERING

\section{CALCULATIONS}

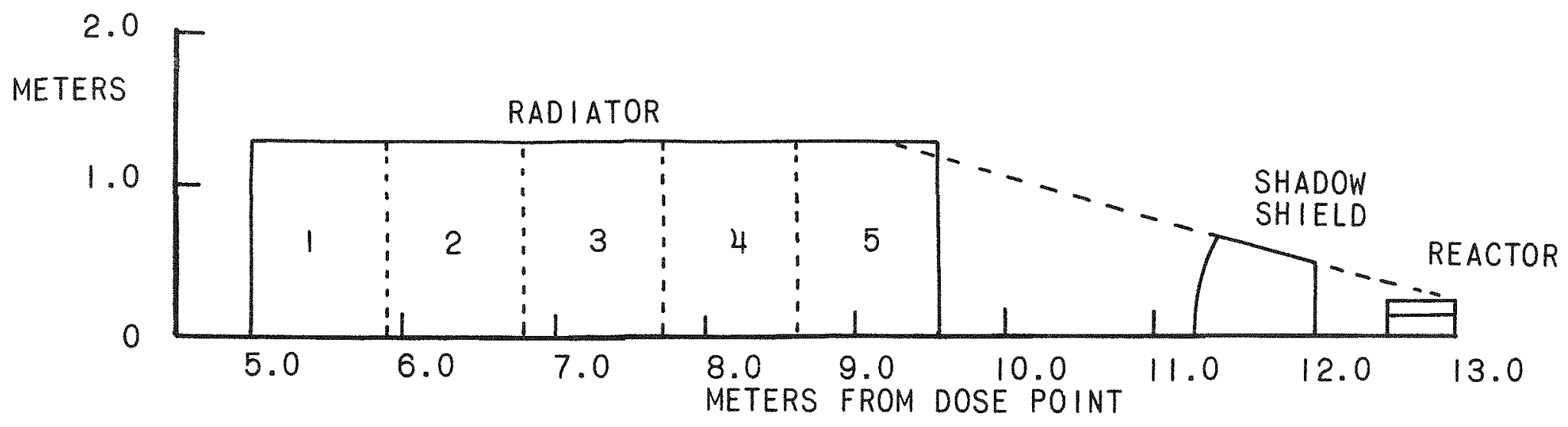

$\therefore \ldots .:$

$:$

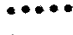

$\therefore$

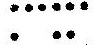

$\therefore \because$

$\therefore$

$\because \cdots$

$\therefore \ldots$

$\vdots$.

$\therefore .$. 


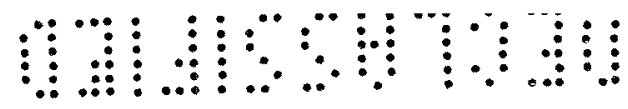

win

HEATING RATE IN Li ${ }^{7}$ H PLUS NATURAL LiH SHIELD REFERENCE DESIGN 2 REACTOR AT 2 MW

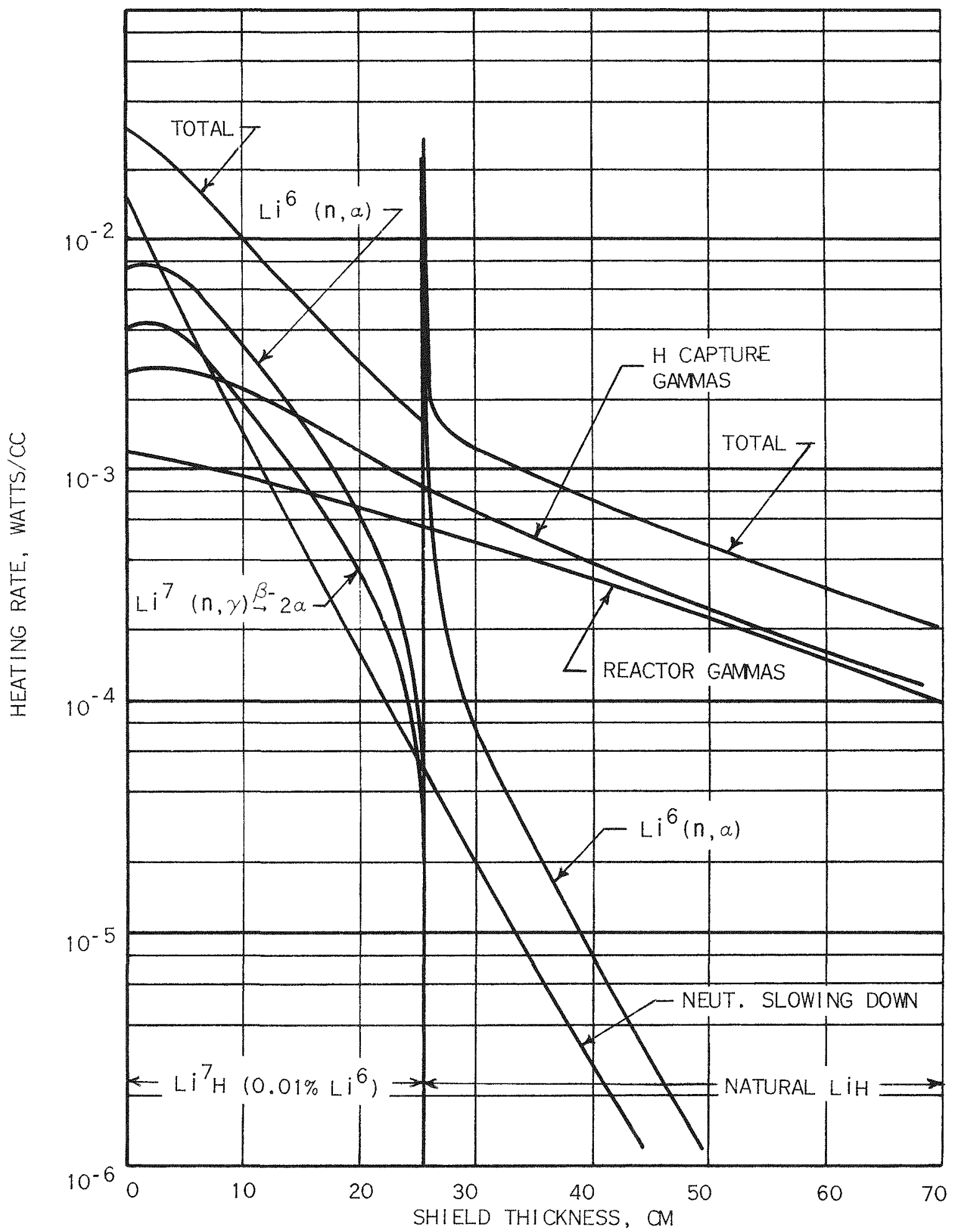




\section{EXPERIMENTAL ARRANGEMENT}

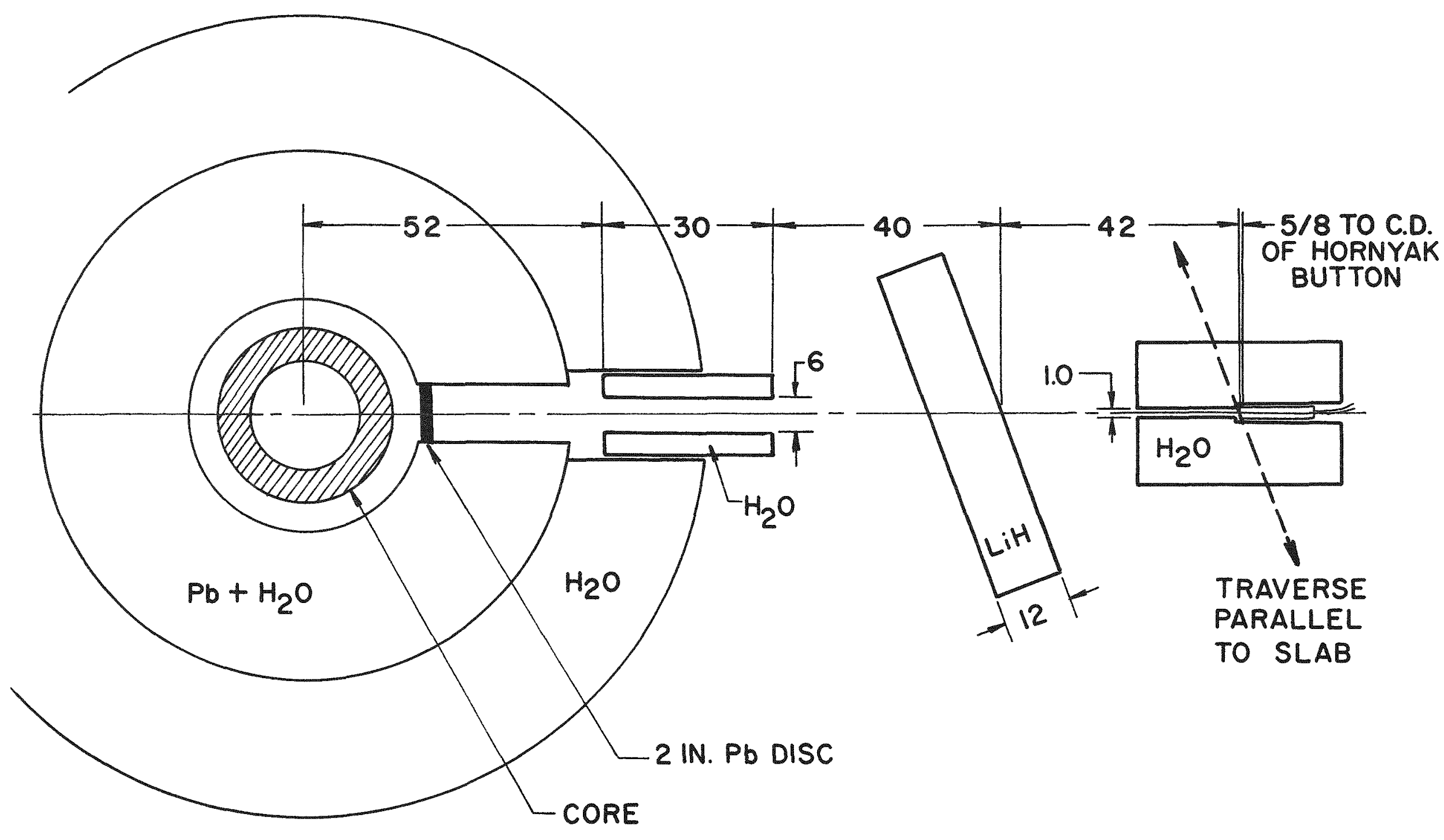

$\therefore: \cdots:$

$\vdots: . .:$

:....

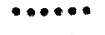

$\because$

$\therefore$

$\because \because$

.......

$\therefore \ldots$ 


\section{SNAP-50 FUEL ELEMENT OPERATION GOALS}

10,000 HOURS, 1900F-2200F CLAD SURFACE

CLADDING:

RESIST LI CORROSION

CONTAIN 20\% FISSION GAS RELEASE (140 pSi) WITH <2\% DIAMETRAL GROWTH WITHSTAND >2\% DIAMETRAL GROWTH WITHOUT ENTERING THIRD STAGE CREEP.

BARR IER: PREVENT GROSS REDUCTION OF FUEL TO U BY CLADDING.

FUEL:

RELEASE <20\% FISSION GAS AND UNDERGO <8\% DENSITY CHANGE WITH I\% BURNUP. RESIST GROSS REDUCTION TO METALLIC U IN THE EVENT OF CLAD FAILURE. RESIST GROSS REDUCTION TO U IN THE EVENT OF BARRIER FAILURE 


\section{STATUS OF UN AND UC PROPERTY STUDIES AT CANEL}

\begin{tabular}{|c|c|c|c|c|}
\hline & & UC & & UN \\
\hline COEFFICIENT OF EXPANSION & 100F & TO & $2800 F$ & IN PROGRESS \\
\hline HEAT CAPACITY & $600 F$ & TO & $2200 \mathrm{~F}$ & $600 \mathrm{~F}$ TO $2400 \mathrm{~F}$ \\
\hline THERMAL CONDUCTIVITY & $1650 F$ & TO & $2900 \mathrm{~F}$ & IN PROGRESS \\
\hline VAPOR PRESSURE AND THERMDDYNAMICS & $3600 F$ & TO & $4000 \mathrm{~F}$ & $3050 F$ TO $3600 F$ \\
\hline HOT HARDNESS & $1800 \mathrm{~F}$ & TO & $2600 \mathrm{~F}$ & $1800 \mathrm{~F}$ TO 2600 \\
\hline
\end{tabular}




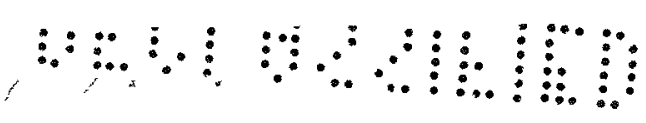

\section{ADVANCED MATERIALS PROGRAM}

\section{FABRICATION}

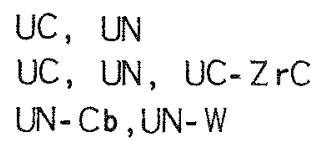

\section{DEVELOP TECHNI QUES}

SYNTHESIS

SINTERING AND HOT PRESSING

COMPATIBILITY

ESTABLISH BASIC BEHAVIOR

$\mathrm{Cb}$ AND $\mathrm{Cb}-1 \mathrm{Zr}$

(W) $\mathrm{Cb}-1 \mathrm{Zr}, \mathrm{W}$

(Ta) $\mathrm{Cb}-1 \mathrm{Zr}$

\section{PROPERTIES}

HEAT CAPACITY

COEFFICIENT OF EXPANSION

HOT HARDNESS

THERMAL CONDUCTIVITY

VAPOR PRESSURE, THERMODYNAMICS

IRRADIATION

ESTABLISH PREL IMINARY IRRADIATION BEHAVIOR DEVELOP AND FABRICATE 3500 F CAPSULE

OTHER

INVESTIGATE EFFECT OF RE ON ELECTRON EMISSION AND $\emptyset$

ESTABLISH THERMAL DIFFUSION BEHAVIOR

ESTABLISH PHASE RELATIONSHIPS a....:

$\therefore:$

(n)

$\therefore$

(n)

$\because \cdots$

$\because \because$

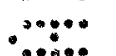

$\vdots$

$\because \cdots$

$\cdots$

a....? 


\section{URANIUM CARBIDE - COLUMBIUM-1 ZIRCONIUM COMPATIBILITY COUPLE}

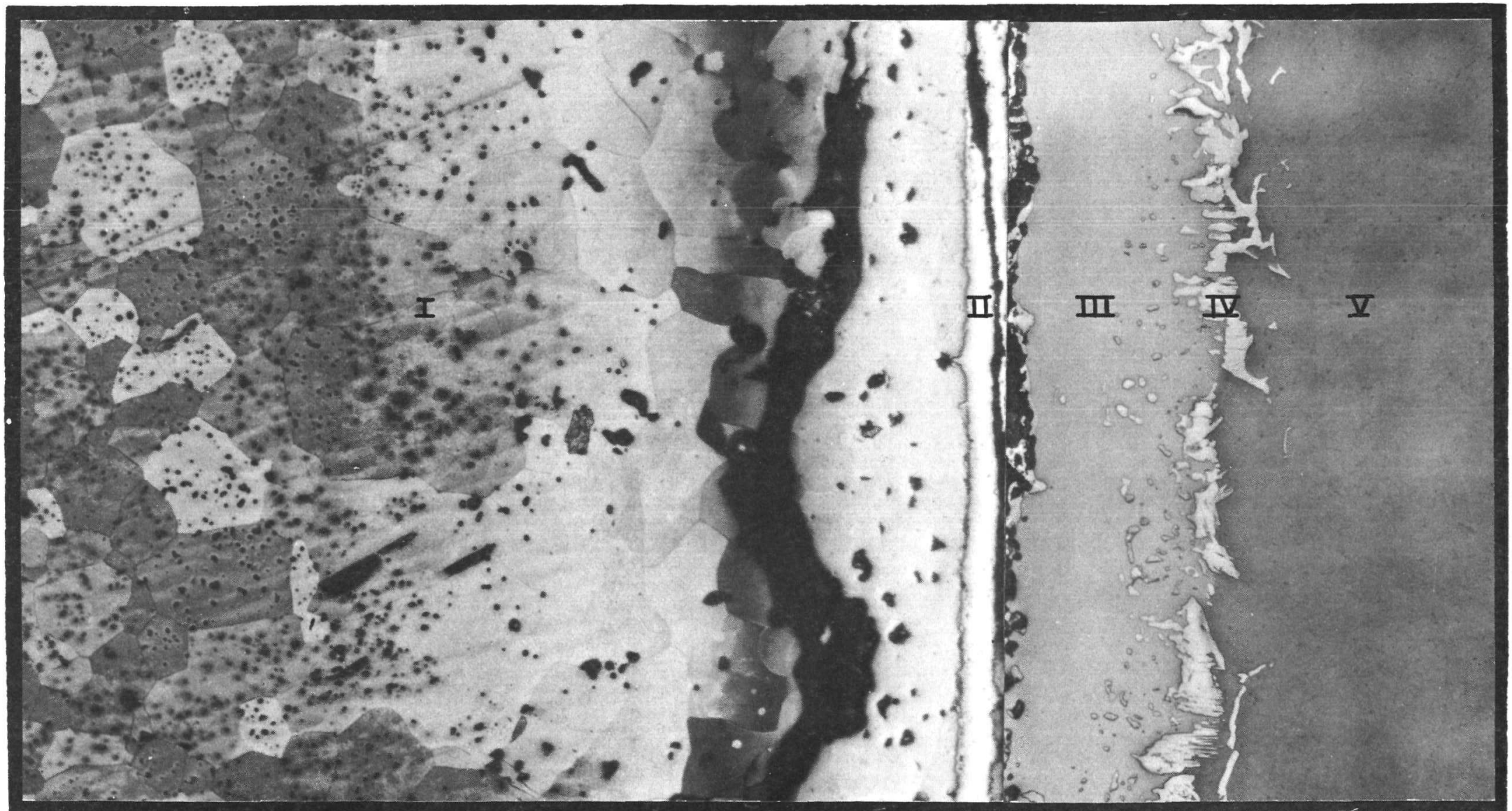




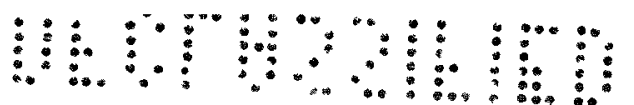

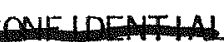

\section{$U_{2} C_{3}$ MIGRATION IN HYPERSTOICHIOMETRIC UC FUEL TEST SPECIMENS (2200F, LITHIUM ENCAPSULATED)}

\begin{tabular}{|c|c|c|c|c|c|}
\hline Specimen No. & $\begin{array}{c}\text { \% Carbon } \\
\text { Pretest } \\
\end{array}$ & Test Time, $\mathrm{hr}$ & Liner & $\begin{array}{c}\text { Carbon } \\
\text { Migration } \\
\end{array}$ & Micro-Probe Fuel \\
\hline $\mathrm{C} 202^{(\mathrm{a})}$ & $\begin{array}{l}4.92 \\
5.08\end{array}$ & 1000 & None & Yes & $\begin{array}{l}-- \\
--\end{array}$ \\
\hline $\mathrm{C} 203^{(\mathrm{a})}$ & $\begin{array}{l}4.92 \\
5.08\end{array}$ & 3000 & None & Yes & $\begin{array}{l}\mathrm{Cb} \\
--\end{array}$ \\
\hline $\mathrm{C} 202^{(\mathrm{a})}$ & $\begin{array}{l}5.21 \\
5.21\end{array}$ & 1000 & Tantalum & None & -- \\
\hline $\mathrm{C} 203^{(\mathrm{a})}$ & $\begin{array}{l}4.92 \\
4.92\end{array}$ & 3000 & Tantalum & None & $\begin{array}{l}-- \\
--\end{array}$ \\
\hline $\mathrm{C} 101^{(\mathrm{a}, \mathrm{b})}$ & $\begin{array}{l}4.85 \\
4.85\end{array}$ & 1000 & None & None & -- \\
\hline $\mathrm{C} 105^{(\mathrm{a}, \mathrm{b})}$ & $\begin{array}{l}4.85 \\
4.85\end{array}$ & 3000 & None & None & -- \\
\hline $170 \mathrm{C}$ & 5.3 & 1000 & None & None & -- \\
\hline $150 \mathrm{C}$ & 5.3 & 1000 & None & None & -- \\
\hline
\end{tabular}

(a) Out-of-pile compatibility test, 2 pins per test

(b) Not lithium encapsulated

(c) Irradiation capsule control test, 3 pins for test 
":

$U_{1.0}$ VERSUS 1 MIL TUNGSTEN/Co- $1 \mathrm{Zr}$

$2200 \mathrm{~F}-4000 \mathrm{HRS}$

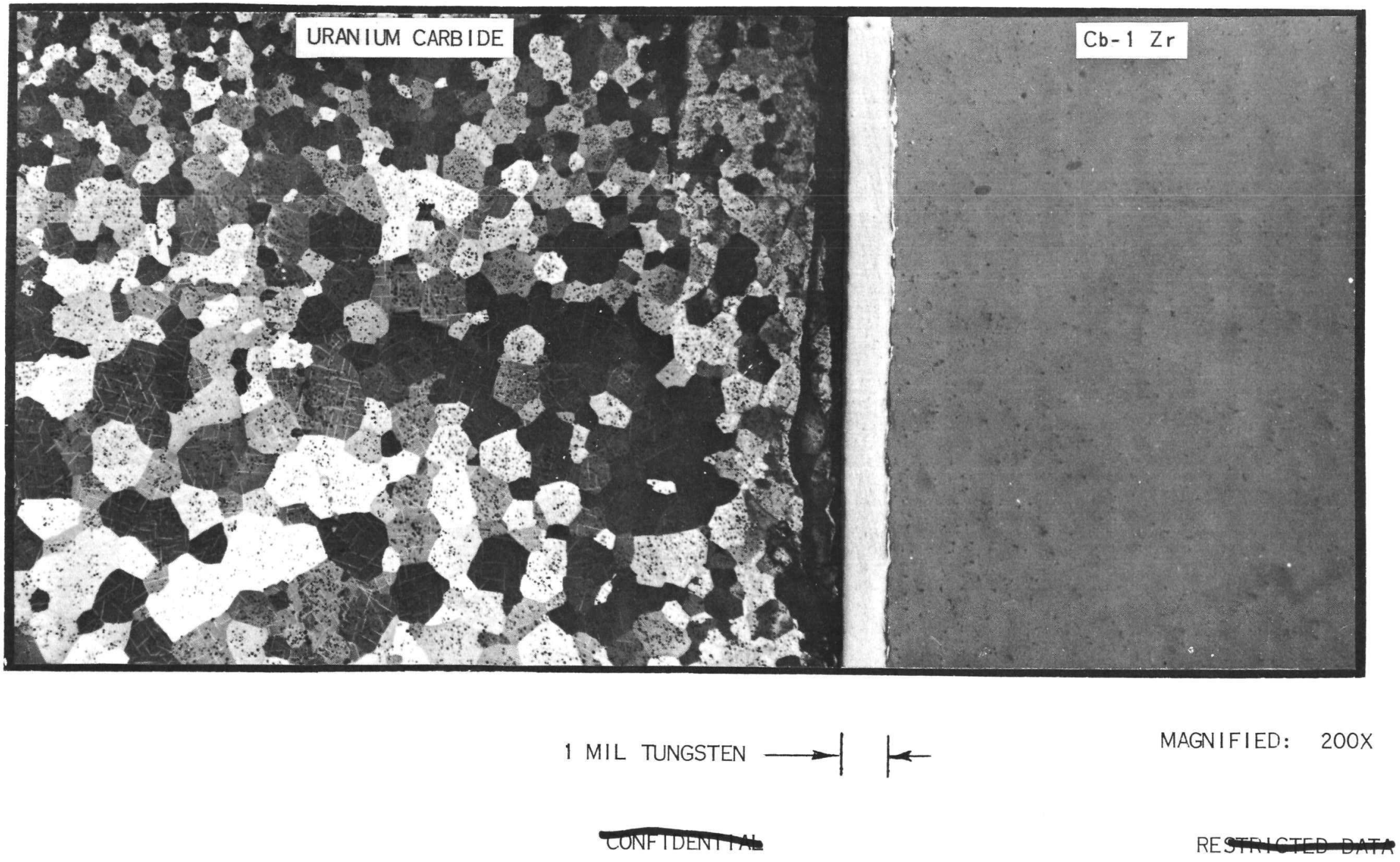

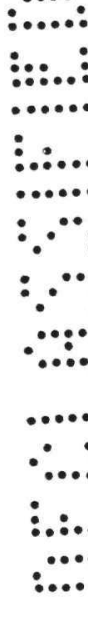




\section{FUEL-CLADDING COMPATIBILITY AT $2200 \mathrm{~F}^{1}$ (SOUND PINS)}

\begin{tabular}{|c|c|c|c|c|c|}
\hline \multirow[b]{2}{*}{ FUEL } & & \multirow{2}{*}{$\begin{array}{l}\text { MAX. TEST } \\
\text { TIME, HR }\end{array}$} & \multicolumn{3}{|c|}{ EFFECT } \\
\hline & BARRIER & & FUEL & BARRIER & $C b-1 \quad \operatorname{~rr}$ CLAD \\
\hline$U c_{1.1}$ & NONE & 9000 & C LOSS & -- & CARBURIZED \\
\hline$U C_{1.1}$ & $\mathrm{Ta}$ & 9000 & C LOSS & CARBURIZED & SL. CARBURIZED \\
\hline$U C_{1.0}$ & W & 6000 & NONE & NONE & NONE \\
\hline UN & NONE & 3000 & NONE & -- & NONE 2 \\
\hline UN & W & 3000 & NONE & NONE & NONE \\
\hline
\end{tabular}

$\therefore: . .:$

$:: \ldots$

(n.....

$\vdots \ldots$

$\because$

$\therefore$

$\because \because$

(n)

$\therefore .$.

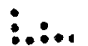

$\therefore: .$. 


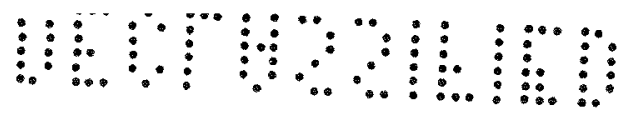

CONFTOETHAL

FUEL-CLADDING COMPATIBILITY AT $2200 \mathrm{~F}^{1}$ (DEFECTED PINS)

\begin{tabular}{|c|c|c|c|c|c|c|}
\hline FUEL & BARRIER & $\begin{array}{l}\text { MAX. TEST } \\
\text { TIME, HR }\end{array}$ & FUEL & $\frac{\text { EFFECT }}{\text { BARRIER }}$ & $\begin{array}{c}C b-1 \mathrm{Zr} \\
C L A D\end{array}$ & $\begin{array}{c}\text { TIME } \\
\text { U PENETRATE } \\
\text { CLAD (HRS) }\end{array}$ \\
\hline$U c_{1.1}$ & NONE & 9000 & U & $U$ & U & 1000 \\
\hline$U c_{1.1}$ & $\mathrm{Ta}$ & 9000 & $U$ & U & $U$ & 3000 \\
\hline$U C_{1} .0$ & $W$ & 6000 & $U$ & U & $U$ & 5000 \\
\hline UN & NONE & 3000 & NONE & - & $4 \mathrm{MILU}$ & $-\cdots$ \\
\hline UN & W & 3000 & NONE & NONE & NONE ${ }^{2}$ & --- \\
\hline
\end{tabular}

$\therefore$

$:: . .$.

(2...

...... $\therefore$

$\because \cdots$

$\because \because 0^{\prime}$

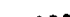

$\therefore$

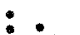

$\cdots$

... 
○.

URANIUM NITRIDE COMPATIBILITY SPECIMEN

$\mathrm{Cb}-1 \mathrm{Zr}$ ALLOY CLAD W BARRIER

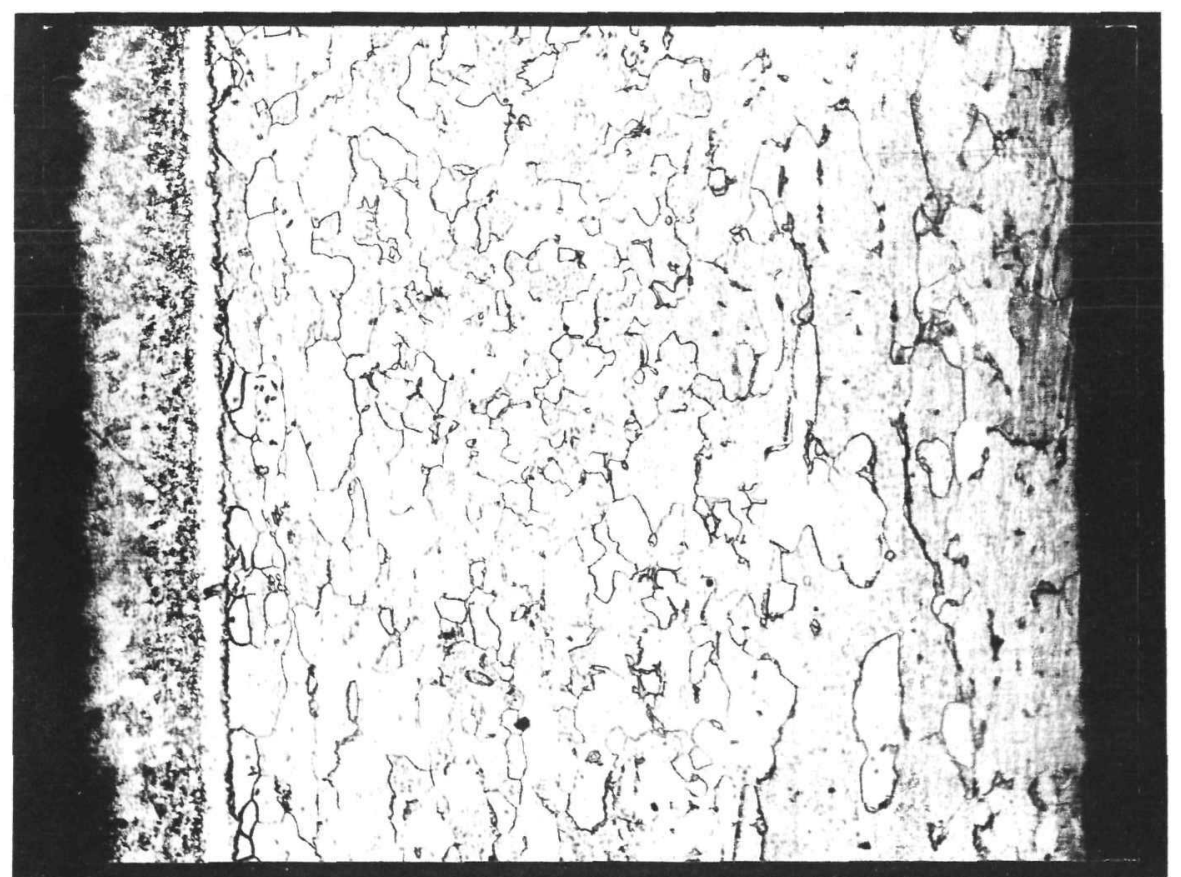

SOUND (AND DEFECTED) PIN

LITHIUM ENCAPSULATED

3000 HOURS

$2400 \mathrm{~F}$

$\because \cdots \cdots:$

$\therefore: \vdots:$

$\because \ldots$

$\therefore . .$.

$\because \because \bullet$

$\because \cdots$

$\because$

$\because \ldots$

$\because \ldots$

$\cdots$ 


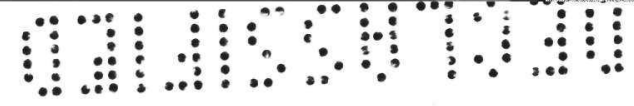

COWIDENTIAL

COMPONENTS - NAK SAMPLER

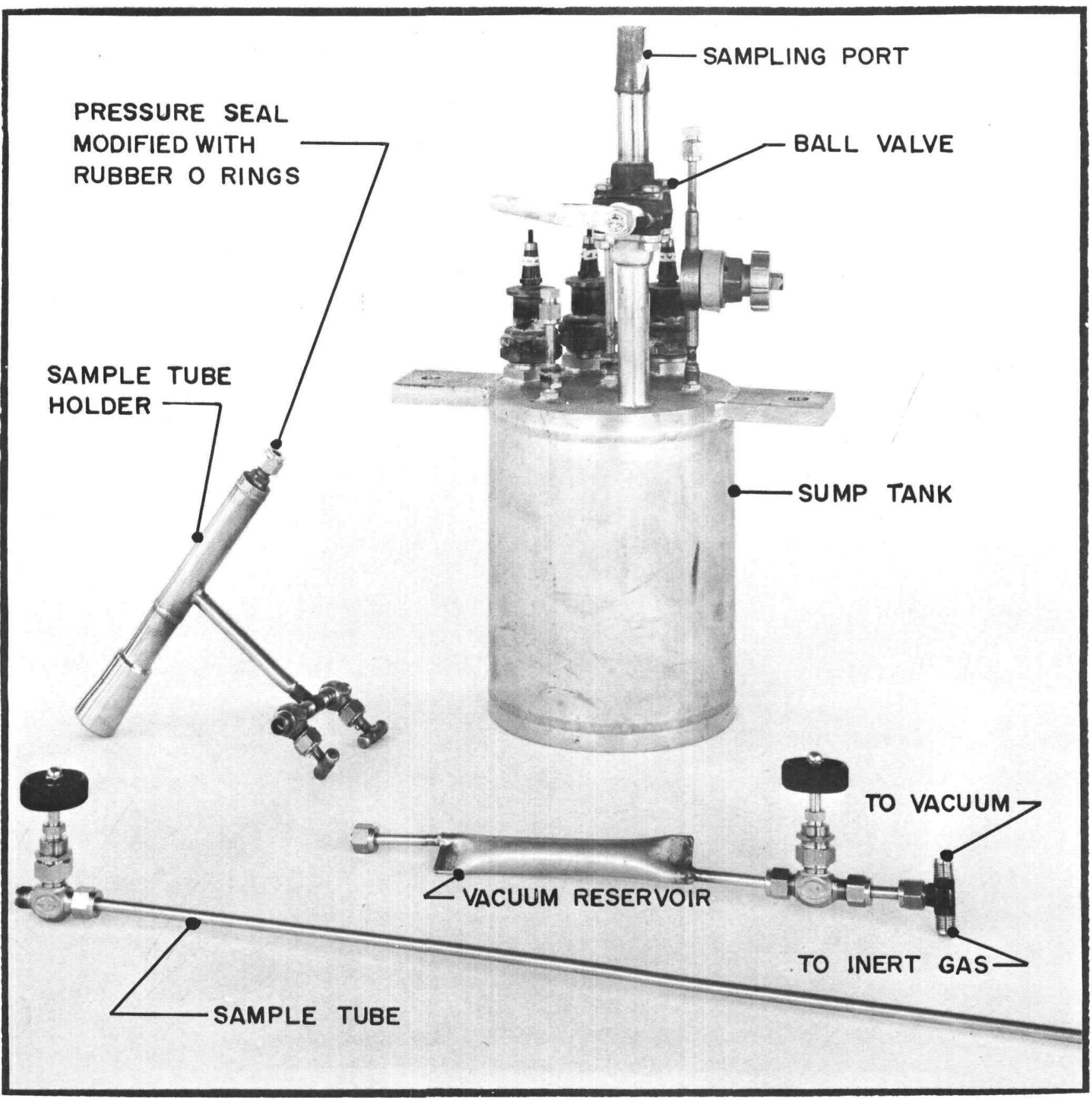




\section{UC AND UN IRRADIATION RESULTS}

$\begin{array}{lcc}\text { NO. OF CAPSULES } & 14 & 8 \\ \text { NO. OF SPECIMENS } & 42 & 24 \\ \text { TEMP. RANGE, F } & & \\ \quad \text { CLADDING SURFACE } & 1850-2225 & 1525-2225 \\ \quad \text { FUEL CENTER } & 2450-2875 & 1675-2850 \\ \text { FISSION BU RANGE, a/O U } & 0.31-1.83 & 0.05-2.34 \\ \text { POWER DENSITY RANGE, KW/CC } & 1.17-1.93 & 0.37-2.22 \\ \text { TIME RANGE, HR. } & 670-3850 & 390-3000 \\ \text { NO. SPECIMENS FAILED } & 12 & 0\end{array}$

$\because \cdots:$

$:: . .:$

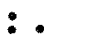

$+\cdots$

$\because \because$

$\because \because$ $\because$

(.....:

$\therefore .$.

$\vdots: \ldots$ $\therefore \ldots:$ 
आआयाओ

FISSION GAS RELEASE VERSUS BURNUP FOR URANIUM CARBIDE

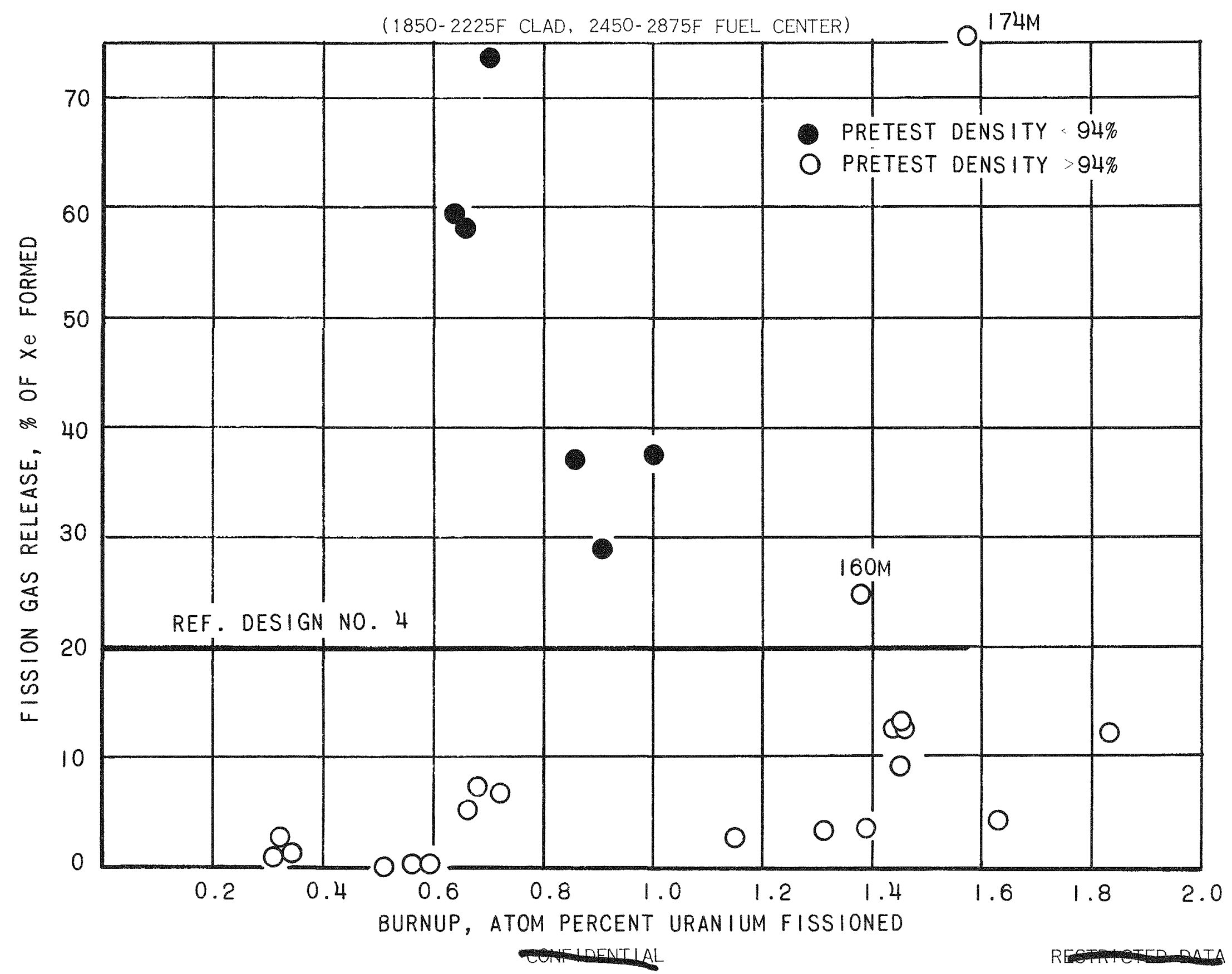

$\therefore: \cdots:$

$:: . .:$

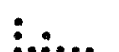

(......

$\therefore \because$

$\because \because$

$\because \because:$

.......

$\therefore \therefore$

$\therefore$ $\therefore: \cdots:$ 
mblatom

FISSION GAS RELEASE VERSUS BURNUP FOR URANIUM NITRIDE
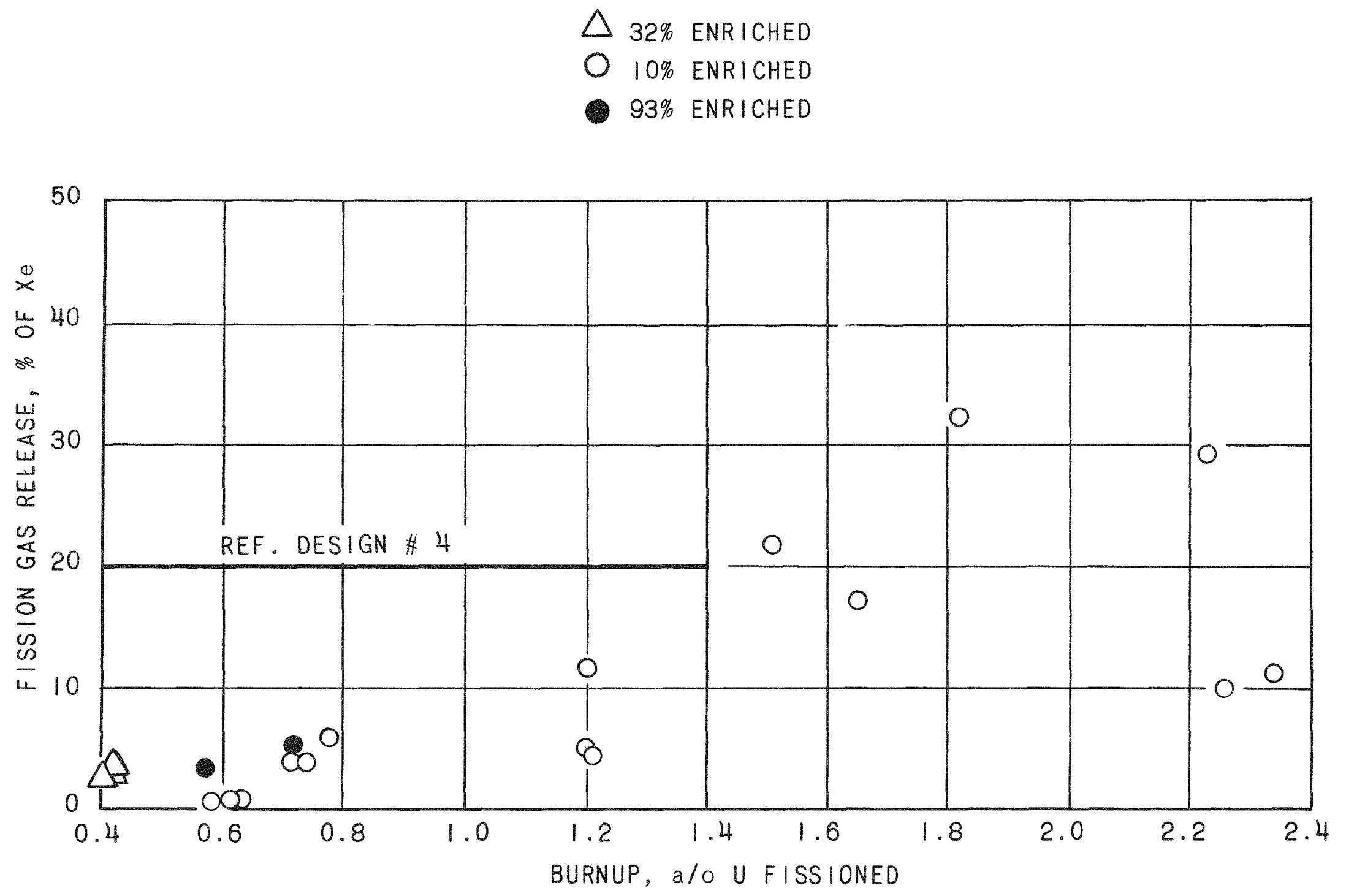

$\therefore: \cdots:$

::..:

:.:...

$\because \because$

$\because \because$

$\ldots$

.......

$\therefore .$.

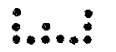

$\therefore: \cdots:$ 
๑)
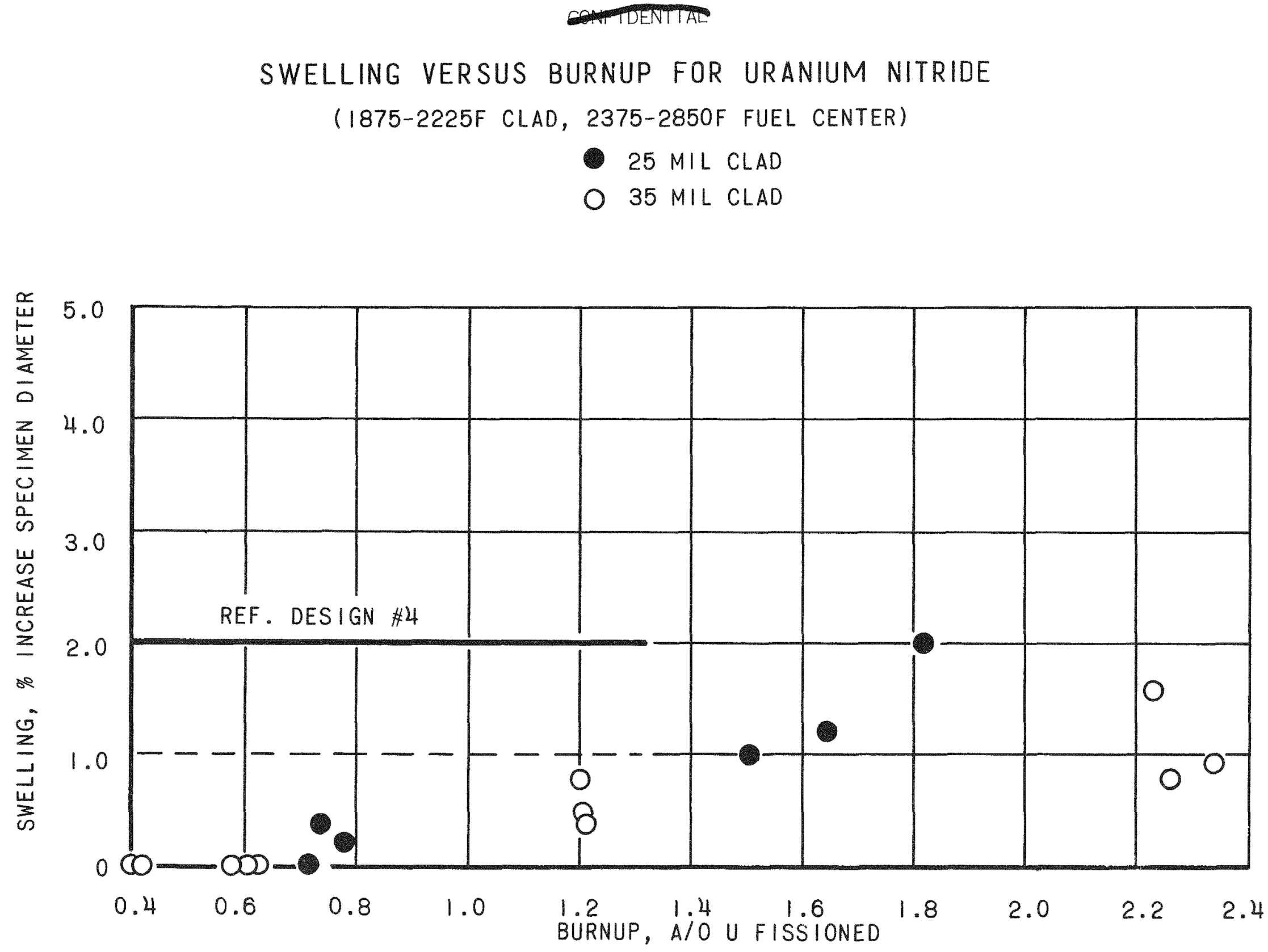

$: \cdots::$

::..:

(n)

:.....

$\because \because$

$\because$

$\because:$

(......

$\therefore . .$.

$\therefore \ldots$ :...:: BURNUP, A/O U FISSIONED 
औणआया

FUEL DENSITY DECREASE VERSUS BURNUP FOR URANIUM CARBIDE

(1850-2225F CLAD, 2450-2875F FUEL CENTER)

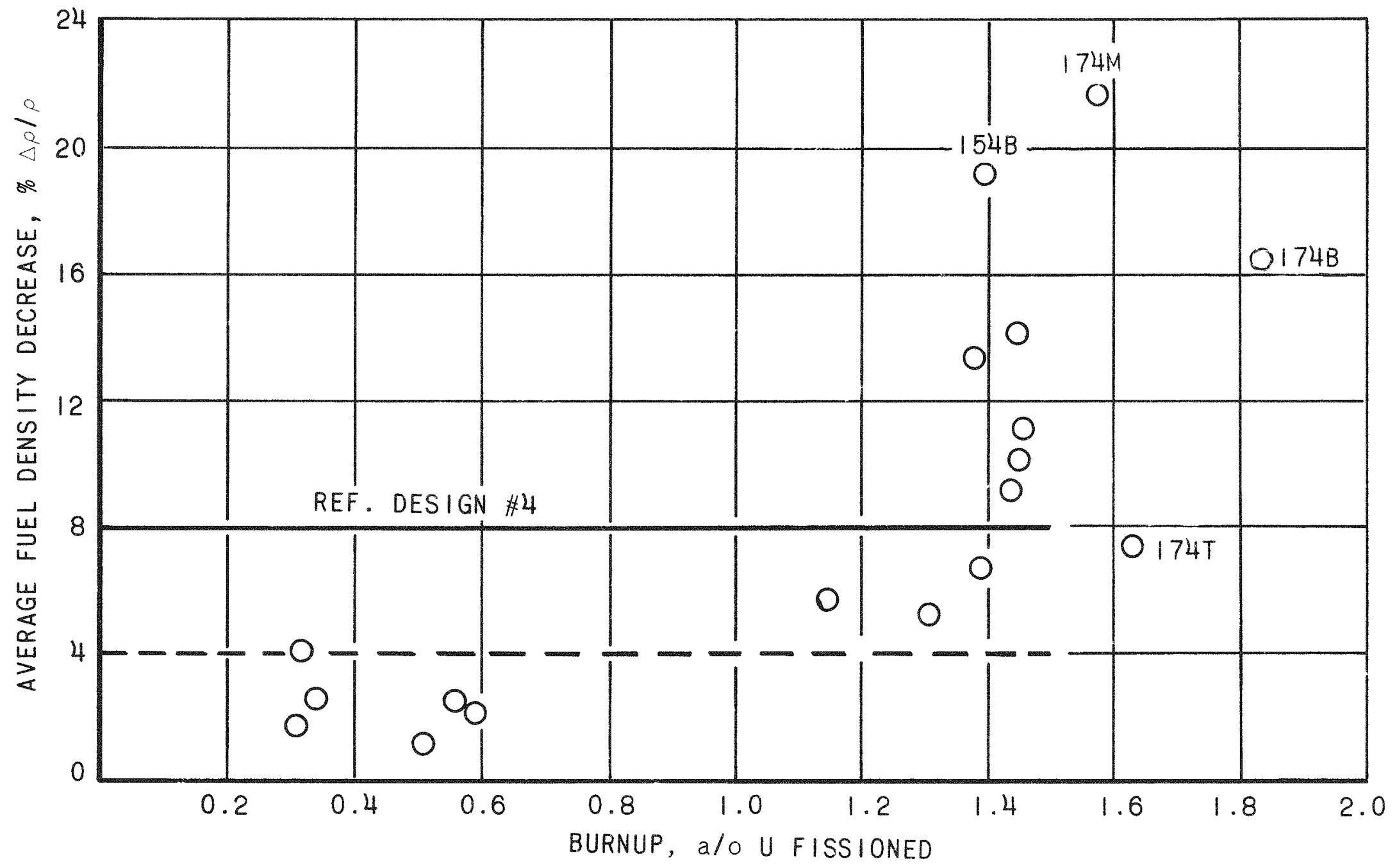

$\therefore: .: 0:$

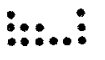

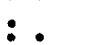

$\therefore$

$\because \because$

$\because \because$

$\because \cdots$

.....:

$\therefore$.

$\therefore$

:::::: 
FISSION GAS RELEASE VERSUS FUEL DENSITY DECREASE FOR URANIUM CARBIDE

O INTERgRANULAR POROSITY

- transgranular porosity

$\therefore . . .:$

$\therefore$

$\ldots . .: 8$

(.....:

$\cdots$

$\because \because$

$\because \because$

$\because \because \%$

$\vdots \ldots$

$\therefore \ldots$

$\vdots . .$.

$\therefore \ldots$ :

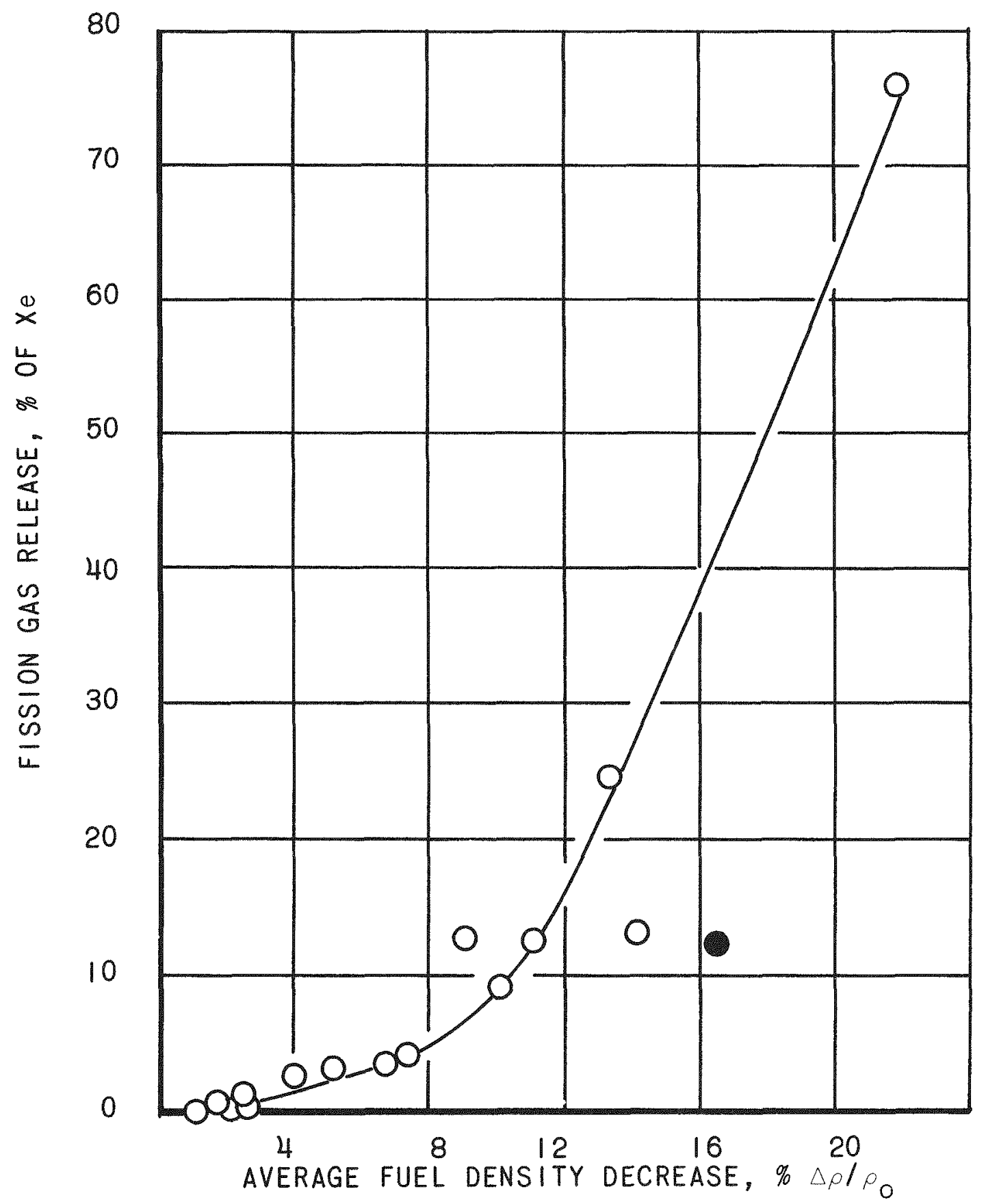

COATL 
FISSION GAS RELEASE VERSUS FUEL DENSITY DECREASE FOR URANIUM NITRIDE

$\square$ BASED ON CS BU

O BASED ON MS OR AVG. BU

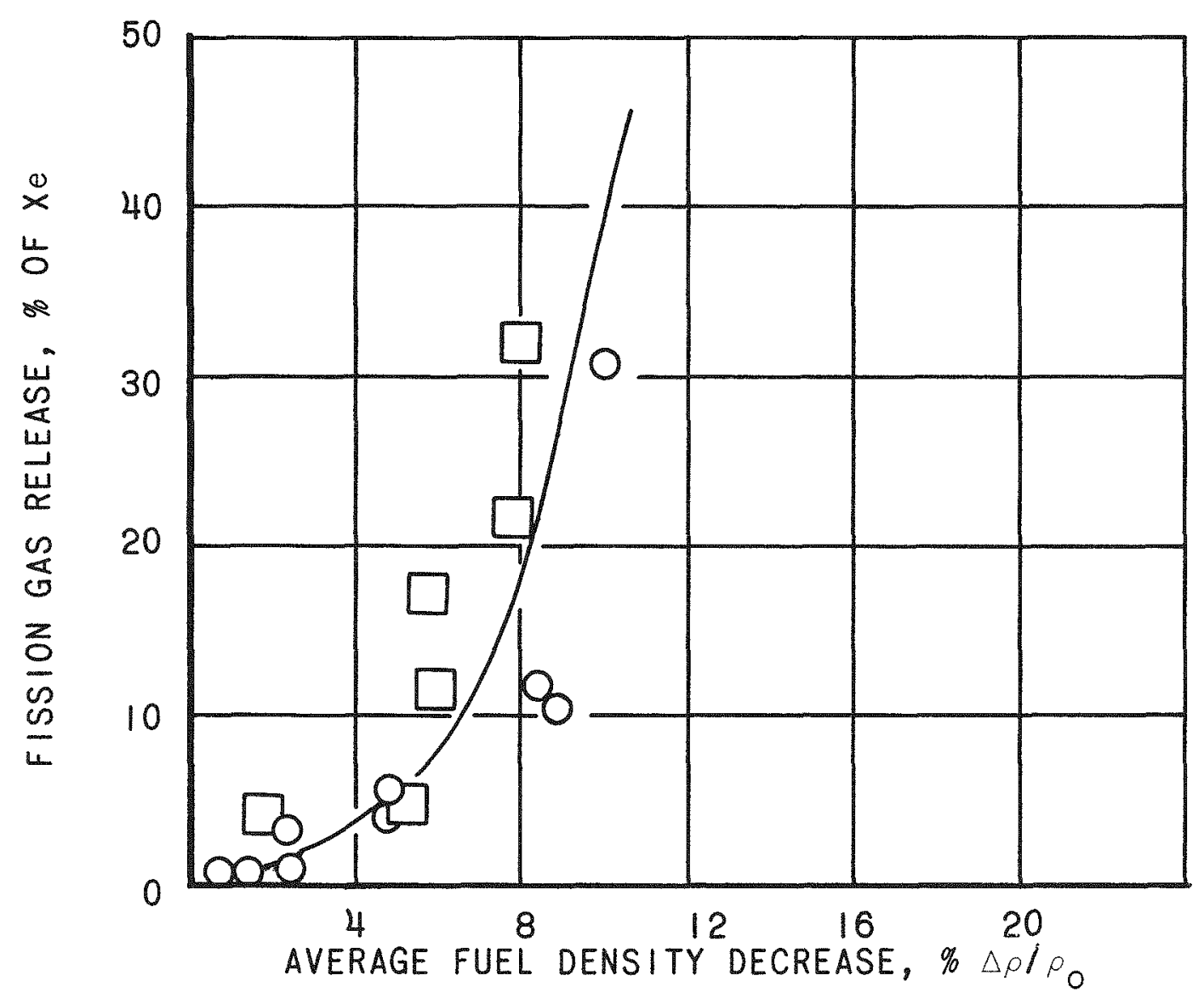

$\therefore: \cdots:$

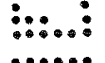

$\vdots \ldots$

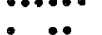

$\because$

$\because$

$\because:$

.....

$\therefore .$.

$\therefore$

$\therefore \ldots$ 


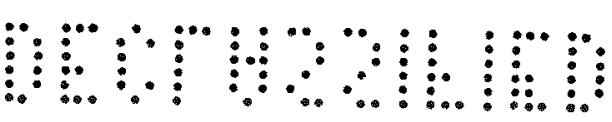

FUEL DENSITY DECREASE VERSUS BURNUP FOR UC AND UN

O UC (CANEL)

- UN

$\triangle$ UC (UNC 5080)

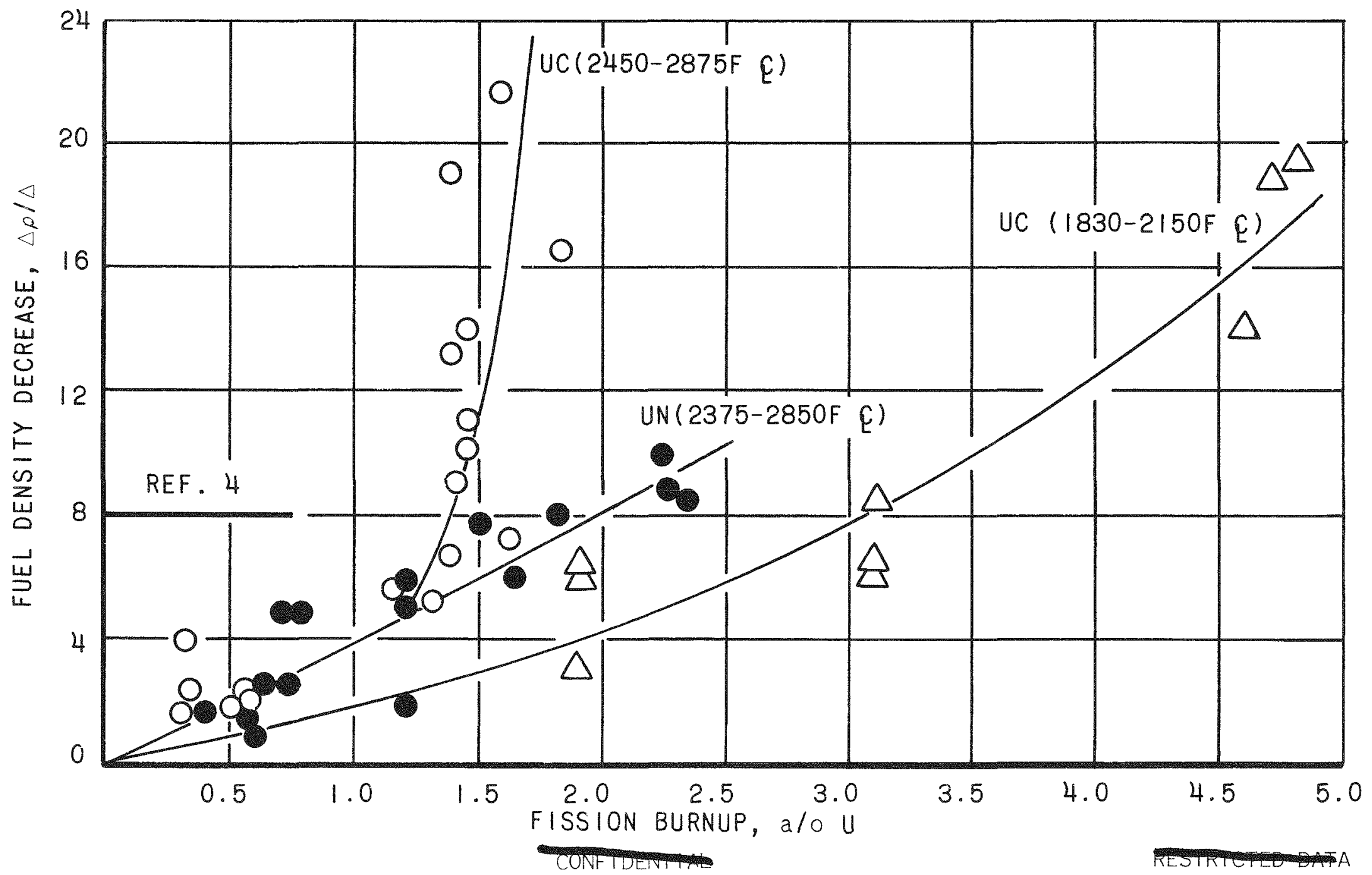

$\therefore:$

::...:

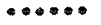

:......

$\because$

$\because \because$

$\because$

$\because \because:$

......

$\therefore \therefore$

$\therefore$

$\therefore$ 
आִ

GONFLOENTIAL

FUEL DEVELOPMENT PROGRAM GOALS

DEMONSTRATE UC AND UN CAPABILITY UNDER 2 MW SNAP-50 CONDITIONS TO BURNUP OF $1.5 \mathrm{a} / 0 \mathrm{U}$.

EXTEND BURNUP LIMITATIONS OF UC AND UN BASE FUELS BEYOND 1.0 a/o U.

MINIMIZE FUEL PIN RUPTURE AND BARRIER FAILURE EFFECTS.

DEVELOP IMPROVED CLADDING FOR UC AND UN. 
आִ

CONEIDCNTH

\section{SNAP-50 FUELS IRRADIATION CONDITIONS}

\begin{tabular}{|c|c|c|}
\hline & REACTOR DESIGN & IRRADIATION TEST \\
\hline TEMPERATURE (F) & 2200 & 2200 \\
\hline TIME (HRS) & TO 10,000 & TO 10,000 \\
\hline FLUX & FAST & THERMAL \\
\hline ENRICHMENT $\% U^{235}$ & 85 & 10 \\
\hline POWER DENSITY $(\mathrm{Kw} / \mathrm{CC})$ & 0.4 & 0.4 \\
\hline
\end{tabular}

$\therefore: .::$

$\vdots: . . .:$

(n....

$\vdots \ldots$

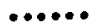

$\therefore \because$

$\because \because$

$\because \because:$

$\ldots$

$\therefore \ldots$

$\vdots . .$.

$\cdots$. 
SNAP-50 UC AND UN IRRADIATION SUPPORT PROGRAM

\begin{tabular}{|c|c|c|c|c|c|c|c|c|}
\hline CLAD TEMP, $F$ & & 2000 & & & 2200 & & CLAD & BARR IER \\
\hline TIME, HRS & 3000 & 6000 & 10,000 & 3000 & 6000 & 10,000 & & \\
\hline BURNUP a/o U & 0.45 & 0.9 & 1.5 & 0.45 & 0.9 & 1.5 & & \\
\hline TOTAL NO. OF CAPSULES & 4 & 4 & -- & 4 & 4 & -- & $\mathrm{Cb}-1 \mathrm{Zr}$ & 2 AND 5 MIL V \\
\hline (DATE) & $(10 / 64)$ & $(4 / 65)$ & -- & $(12 / 64)$ & $(6 / 65)$ & -- & & \\
\hline & 4 & 4 & 4 & 4 & 4 & 4 & $\begin{array}{l}\mathrm{Cb}-1 \mathrm{Zr}- \\
0.6 \mathrm{C}^{-}\end{array}$ & 5 MIL W \\
\hline & $(5 / 65)$ & $(11 / 65)$ & $(8 / 66)$ & $(7 / 65)$ & $(12 / 65)$ & $(9 / 66)$ & & \\
\hline
\end{tabular}

$\therefore \ldots:$

$\vdots:: . . \vdots$

$\vdots \ldots$ $\cdots$ $\because \because$ $\because \because$ $\because \because:$ $\ldots$ $\therefore \ldots$ $\vdots . . .$. $\therefore: \cdots:$ 


\section{BURNUP LIMIT EXTENSION PROGRAM}

(2200F CLADDING SURFACE TEMPERATURE)

\begin{tabular}{|c|c|c|c|c|c|c|c|}
\hline & PURPOSE & FUEL & $\begin{array}{l}\text { NO. } \\
\text { CAPSULES } \\
\end{array}$ & $\begin{array}{l}\text { POWER } \\
\text { DENSITY } \\
(\mathrm{KW} / \mathrm{CC}) \\
\end{array}$ & $\begin{array}{l}\text { TIME, } \\
(H R) \\
\end{array}$ & $\begin{array}{l}\text { DESIGN } \\
\text { BURNUP } \\
\text { a/OU } \\
\end{array}$ & DATE \\
\hline \multirow[t]{2}{*}{ ALLOY } & \multirow[t]{2}{*}{ STRENGTHEN ING } & $U C-10 \mathrm{~m} / \mathrm{o} \mathrm{ZrC}$ & $2^{*}$ & 1.5 & 2500 & 1.5 & $1 / 65$ \\
\hline & & $U C-1 w / 0 w$ & $2^{*}$ & 1.5 & 2500 & 1.5 & $2 / 65$ \\
\hline DENSI & TY VARIATIONS & $81-93 \%$ DENSE UC & $4^{*}$ & 1.5 & 2500 & 1.5 & $2 / 65$ \\
\hline \multirow[t]{3}{*}{ MEAT } & \multirow[t]{3}{*}{ LENGTH VARIATIONS } & \multirow{3}{*}{$\begin{array}{l}\text { UC AND UN, } \\
\text { L/D }=18\end{array}$} & \multirow[t]{3}{*}{16} & \multirow[t]{3}{*}{0.4} & 3000 & 0.3 & $10 / 65$ \\
\hline & & & & & 6000 & 0.9 & $5 / 66$ \\
\hline & & & & & 10,000 & 1.5 & $4 / 66$ \\
\hline \multirow{3}{*}{\multicolumn{2}{|c|}{ FUEL GEOMETRY }} & \multirow{3}{*}{$\begin{array}{l}\text { UC AND UN WITH } \\
\text { CENTER HOLE, } \\
\text { L/D }=18\end{array}$} & \multirow[t]{3}{*}{16} & \multirow[t]{3}{*}{0.4} & 3000 & 0.3 & $10 / 65$ \\
\hline & & & & & 6000 & 0.9 & $5 / 66$ \\
\hline & & & & & 10,000 & 1.5 & $4 / 66$ \\
\hline
\end{tabular}

*AT NRTS 


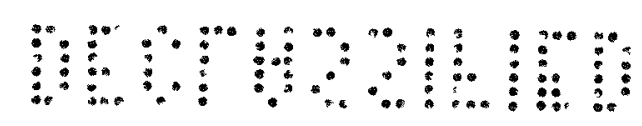

CONFIDENTIAL

\section{FUEL-CLAD COMPATIBILITY AND BARRIER EVALUATION PROGRAM}

$\begin{array}{lccc} & \text { NO. OF TESTS } & & 10,000 \text { HR. DATA } \\ \text { EFFECT OF Li ON FUEL-CLAD COMPATIBILITY } & 130^{*} & 6 / 64-6 / 65 \\ \text { EVALUATION OF COATED FUELS } & 32 & 12 / 65 \\ \text { ADVANCE ALLOY EVALUATION } & & 12 / 65 \\ \text { TANTALUM-BASE ALLOYS } & 20 & 3 / 65 \\ \text { TUNGSTEN-BASE ALLOYS } & 8 & \\ & * 40 \text { IN TEST }\end{array}$

COALENTIAI

REGTI

$\therefore: \cdots:$

::..:

(n.....

$\therefore$ $\therefore$

$\because$

$\ldots$

$\ldots$

$\therefore \ldots$

$\therefore$ $\therefore \ldots:$ 
mentem

\section{OONLIL \\ FUEL CLAD OBJECTIVES}

FABRICABLE (HIGH QUALITY, WELDABLE)

STRONG ( 1500 PSI/2200F/10,000 HRS RUPTURE)

DUCTILE (8\% RUPTURE DUCTILITY)

COMPATIBLE WITH LITHIUM (2200F/10,000 HRS)

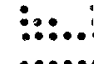

$\vdots . .$.

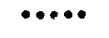

$\because \cdots$

$\because$

$\because \because$

$\therefore \dot{0}$

¿...

$\because \ldots$ $\therefore \ldots$ 


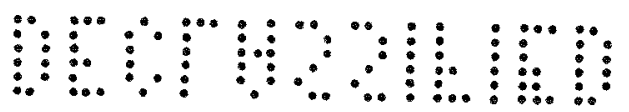

CONELDENT

\section{FUEL CLAD MATERIALS}

\section{ALLOY \\ $\mathrm{Cb}-1 \mathrm{Zr}-0.02 \mathrm{C}$ (REFERENCE)}

$\mathrm{Cb}-1 \mathrm{Zr}-0.065 \mathrm{C}$

$\mathrm{Ta}-9.5 \mathrm{~W}-2.5 \mathrm{Hf}-0.01 \mathrm{C}$
EXTRAPOLATED

RUPTURE STRENGTH , PSI

$2200 \mathrm{~F} / 10,000 \mathrm{HR}$

1500

-....

$\vdots . .$.

$\because \cdots$

$\because \because$

$\because \because$

$\because \because$

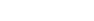

....

$\vdots \ldots$

$\therefore \cdots$ 
STRESS VERSUS RUPTURE TIME

Cb-1 Zr-0.065 C FUEL PIN TUBING

(MANSON-HAFERD PARAMETER)
- 2900F/I HR + 5\% C.W. + 2200F ANN'L (RUPTURE TEST)
O 2900F/I HR + 5\% C.W. + 2200F ANN'L (DISC. TEST)
(1) 2900F/I HR + 2200F ANN'L (RUPTURE TEST)
$\square$ 2900F/I HR + 2200F ANN'L (DISC. TEST)

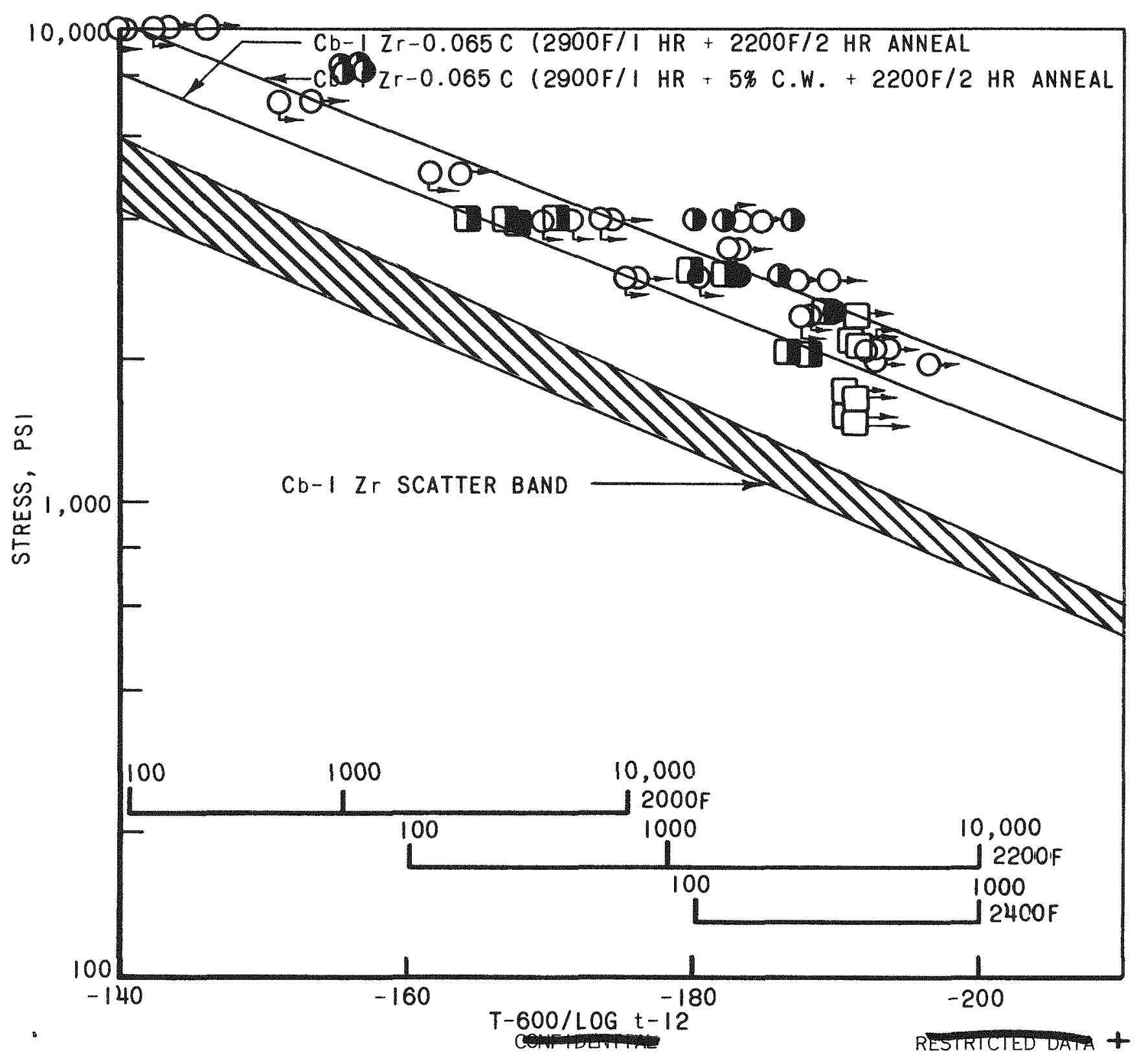




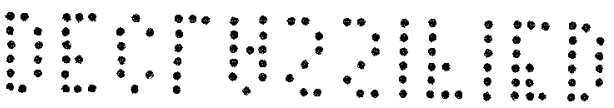

\section{MECHANICAL PROPERTIES EVALUATION PROGRAM}

\section{DETERMINE}

EFFECT OF HEAT TREATMENT

EFFECT OF ASSEMBLY SEQUENCE

THROUGH

RUPTURE TESTS: 2200F Li/100-3000 HRS

SELECT - HEAT TREATMENT SEQUENCE SIZE

DETERMINE

RUPTURE STRENGTH AND CREEP AT DESIGN CONDITIONS

THROUGH

STRESS RUPTURE AND CREEP TESTS OF FUEL CLAD WITH AND WITHOUT BARRIERS

1900F-2600F/1000-10,000 HRS

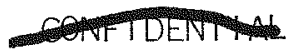

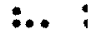

…...

$\vdots \ldots$

.......

$\because \because$

$\because \cdots$

:..:"

......

$\therefore$

,

:.:...

:.....: 


\section{SONEDTLI. \\ STABILITY OF FUEL CLAD IN LITHIUM}

\section{DETERMINE}

EFFECT OF $2200 \mathrm{~F}$ LI ON STABILITY OF CLAD ALLOY IN Cb-1 Zr LOOP

THROUGH

$$
\text { RUPTURE TESTS AND CHEMICAL ANALYSES }
$$

STATUS
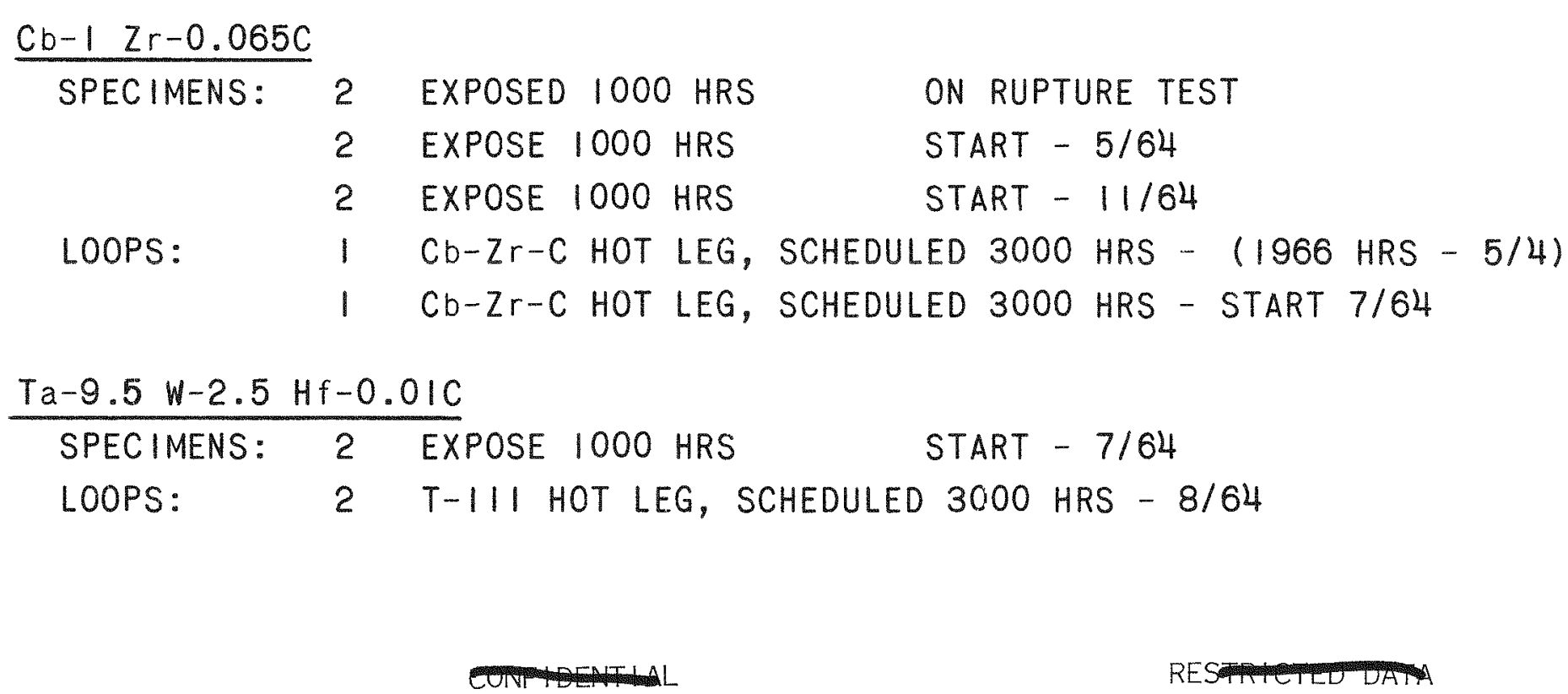
आ4⿴囗十)

STRUCTURAL ALLOY OBJECTIVES

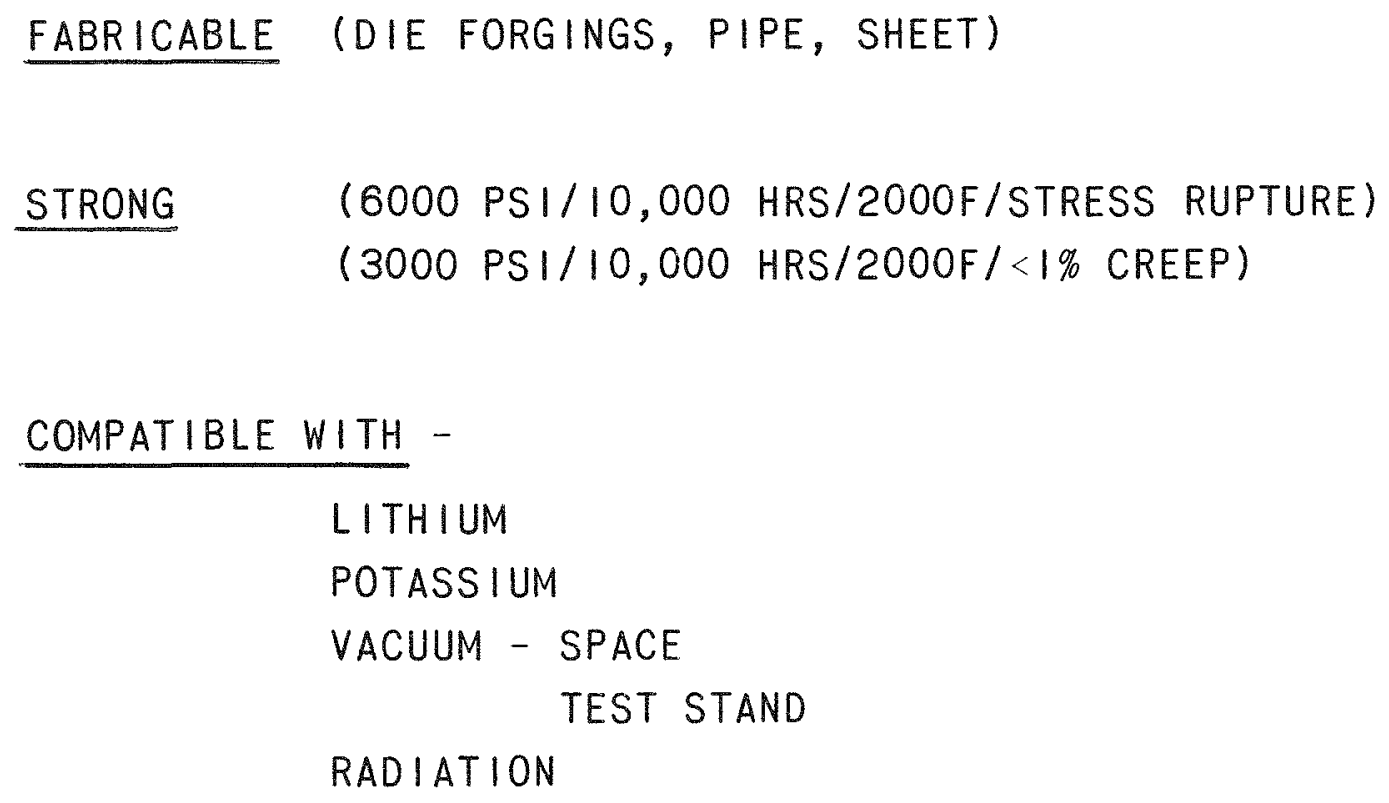




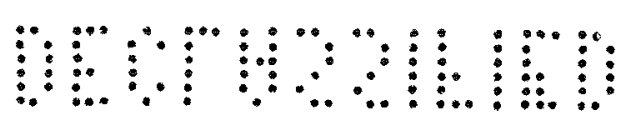

CONFTDETVTTL

\section{STRUCTURAL ALLOY MATERIALS}$$
\mathrm{Cb}-1 \mathrm{ALLOY}
$$$$
\text { (EXTRUDED - 2300F) }
$$$$
\mathrm{Cb}-1 \mathrm{Zr}-0.1 \mathrm{C}
$$$$
\text { (EXTRUDED - 2900F) }
$$$$
\mathrm{Cb}-3 \mathrm{Zr}-0.3 \mathrm{C}
$$ \\ (EXTRUDED - 3IOOF)
}$$
5000
$$

EXTRAPOLATED

$1 \%$ CREEP STRENGTH, PSI $2000 \mathrm{~F} / 10,000$ HRŚ.

2000

5800 $\therefore \ldots:$

$\vdots: . . .:$

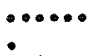

(.....

$\because \cdots$

$\because \because$

$\because \because$

$\because \because:$

(....:

$\therefore \therefore$

$\vdots \ldots$

$\therefore: \cdots:$ 


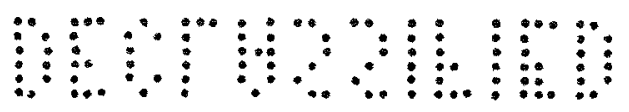

ADVANCED STRUCTURAL ALLOYS

CREEP RATE VERSUS STRESS

(MANSON-HAFERD PARAMETER)

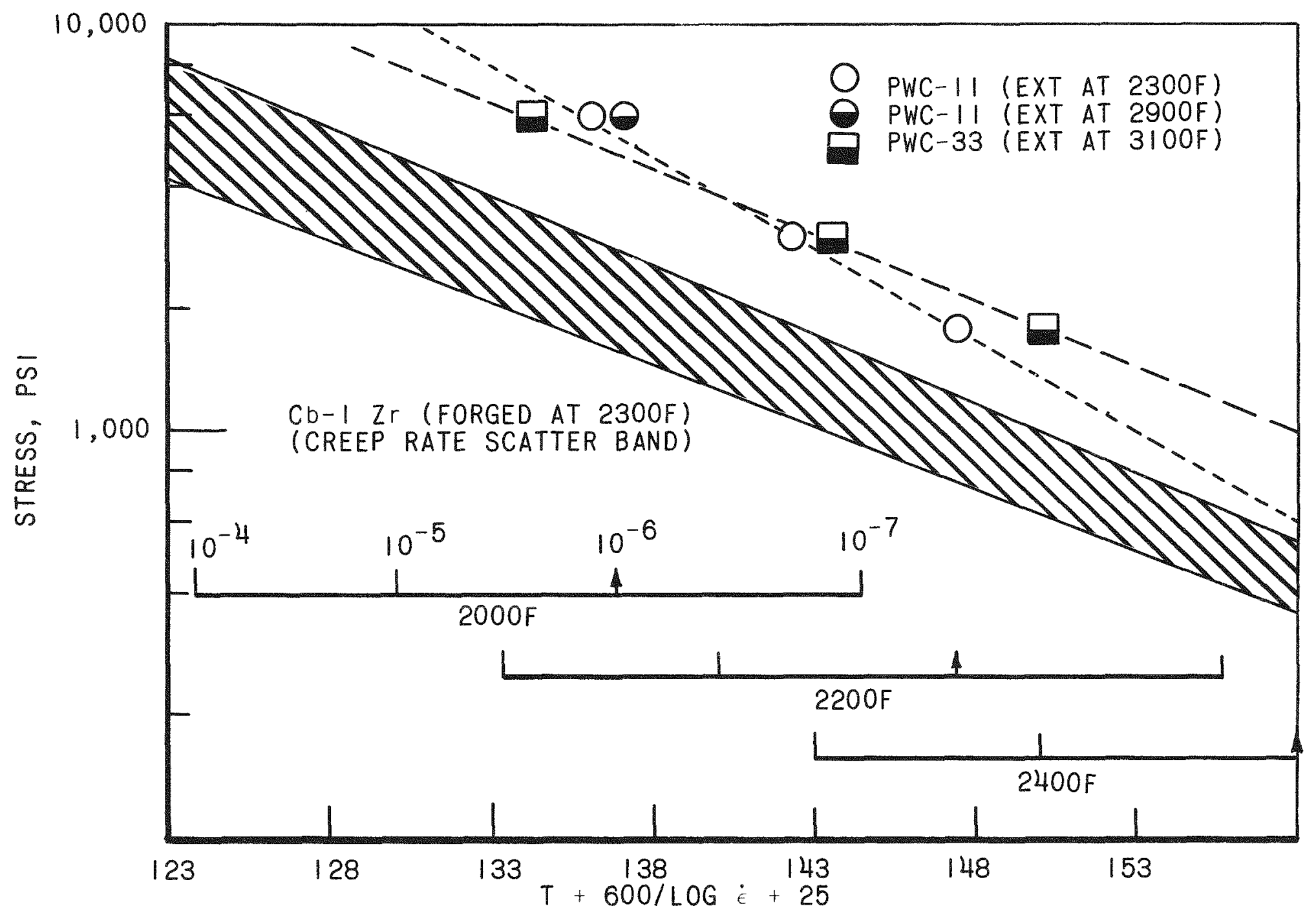

-

\section{$\therefore$}

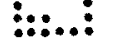

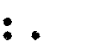

:-....

$\therefore$

$\because \because$

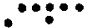

.....:

$\therefore$

$\therefore$

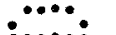


आサan

CONFTDENTTAL

\section{PRESSURE VESSEL TEST PROGRAM}

\section{DEMONSTRATE}

\section{CREEP CORRELATION}

FABRICATION

CONTAINMENT

THROUGH

10 INCH VESSEL TESTS/2000F Li/3000 HRS

IN $10^{-8}$ TORR VACUUM

CONDITIONS

$1-5 \%$ CREEP $\frac{C b-1 Z r}{3} \quad \frac{C b-1 Z r-0.1 C}{3} \quad \frac{C b-3 Z r-0.3 C}{3}$

$\therefore \ldots$

$\vdots: . .: \vdots$

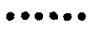

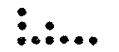

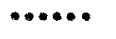

$\because \because$

$\because \because$

$\because \because \cdots$

......

$\therefore \therefore$

$\vdots: . . \vdots$ :...:; 


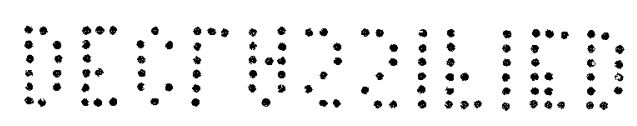

CONTENTTA-

\title{
REQUIREMENTS FOR LITHIUM CONTAINMENT
}

\author{
LOW $\mathrm{O}_{2}$ IN Cb-1 $\mathrm{Zr}$ \\ $<300$ ppm UNANNEALED \\ $<3000 \mathrm{ppm}$ ANNEALED \\ LOW $\mathrm{O}_{2}$ IN ENVIRONMENT \\ $<10^{-7}$ TORR $0_{2} / 10,000$ HRS. \\ $<0.1 \mathrm{ppb} \mathrm{O}_{2}$ IN He/10,000 HRS.
}

DEMONSTRATED BY:

NO ATTACK

CAPSULE TESTS:

$300-3000 \mathrm{ppm} \quad 0_{2}$ (ANNEALED)

PRESSURE VESSEL TESTS:

10,000 HRS - 110 INCH VESSEL

2000F LITHIUM

44 INCH VESSELS

LOOP TESTS:

5000 HRS - I SN-A| COATED

2000/1000F LITHIUM

1000 HRS - I Sn-A| COATED

I Ta WRAPPED

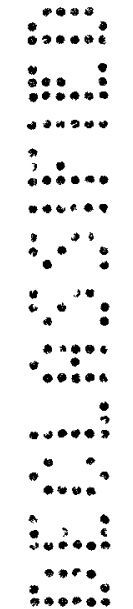


monaln

Gor DC

\section{STRUCTURAL ALLOY CORROSION EVALUATION}

\section{DEMONSTRATE}

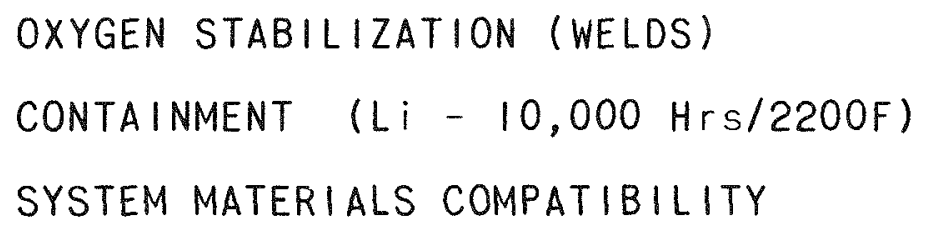

THROUGH

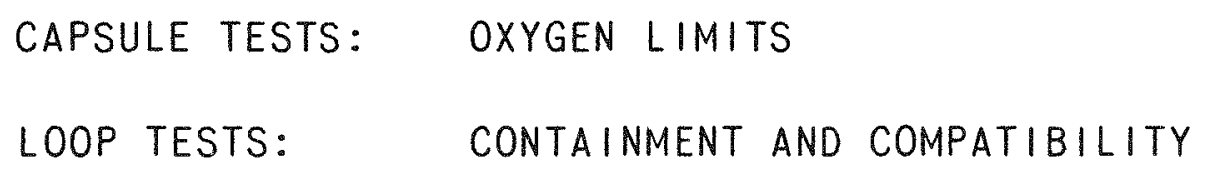

CONDITIONS

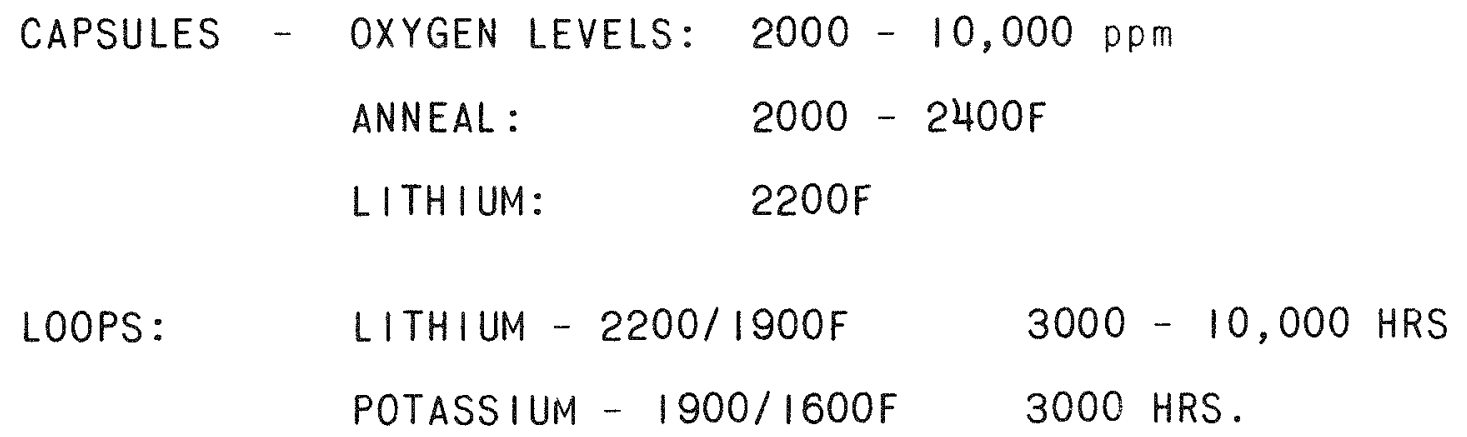


आआया।

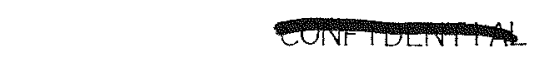

\section{Cb-1 Zr ALLOY IRRADIATION DATA}

\begin{tabular}{|c|c|c|c|c|c|c|}
\hline & TEMP, F & STRESS, PSI & $\begin{aligned} & \text { FLUX } \\
& n v(>1 \text { Mev }) \\
&\end{aligned}$ & , HRS & $\begin{array}{c}\text { DOSE } \\
n v t(>1 \text { Mev }) \\
\end{array}$ & RESULTS \\
\hline $\begin{array}{l}\text { ORNL } \\
\text { RUPTURE TESTS }\end{array}$ & $1800 / 2000$ & $1-3 \times 10^{4}$ & $\sim 10^{13}$ & $1-600$ & $10^{17}-10^{19}$ & $\begin{array}{l}\text { NO SIGNIFICANT } \\
\text { EFFECT }\end{array}$ \\
\hline \multirow{3}{*}{$\begin{array}{l}\text { P\&WA-CANEL } \\
\text { FUEL PIN CLAD- } \\
\text { DING }\end{array}$} & $1500 / 1750$ & -.- & $1.6 \times 10^{13}$ & 10,000 & $3.6 \times 10^{20}$ & \multirow{3}{*}{$\begin{array}{l}\text { NO LOSS IN } \\
\text { - BEND DUCTILITY }\end{array}$} \\
\hline & $1950 / 2180$ & $-\cdots$ & $1.3 \times 10^{13}$ & 2720 & $1.3 \times 10^{20}$ & \\
\hline & $1750 / 2090$ & --- & $2.1 \times 10^{14 *}$ & 1000 & $6.8 \times 10^{20}$ & \\
\hline
\end{tabular}

* etr core

$\therefore: \cdots:$

$\vdots: \ldots$

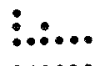

$\because \because$

$\because \because$

$\because \because$

.....

$\therefore \therefore$

$\vdots . .$.

:......: 


\section{STRUCTURAL ALLOY EVALUATION IN VACUUM ENVIRONMENT}

DEMONSTRATE :

ALLOY STABILITY $\left(10^{-7}-10^{-10}\right.$ torr $)$

LITHIUM CONTAMINATION $\left(10^{-7}-10^{-8}\right.$ torr $)$

THROUGH :

1) OXIDATION RATE TESTS

2) CARBON STABILITY TESTS

3) LOOP TESTS

CONDITIONS:

OXIDATION: $10^{-5}-10^{-8}$ torr $02 ; 2000,2200 \mathrm{~F}$ UP TO 1000 HOURS

CARBON STABILITY: $10^{-7}-10^{-9}$ torr $02 ; 2000,2200 \mathrm{~F} ; 500,1000,3000$ HOURS

CONVECTION LOOP: 2000/1900F Li; 1000 HOURS; $1.2 \times 10^{-6}$ torr $0_{2}$ PRESSURE 5000 HOURS; $4.0 \times 10^{-7}$ torr $\mathrm{O}_{2}$ PRESSURE

$\therefore: .:$ :

:::..: (n)

$\vdots . . .$. (n..... $\because \because$ $\because \because$ $\because \because:$ 


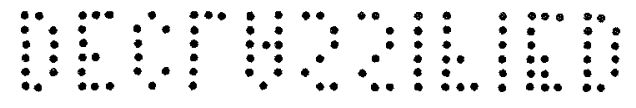

-OATITL

CORRELATION OF OXYGEN PRESSURE WITH CONTAMINATION OF Cb-1 Zr ALLOY

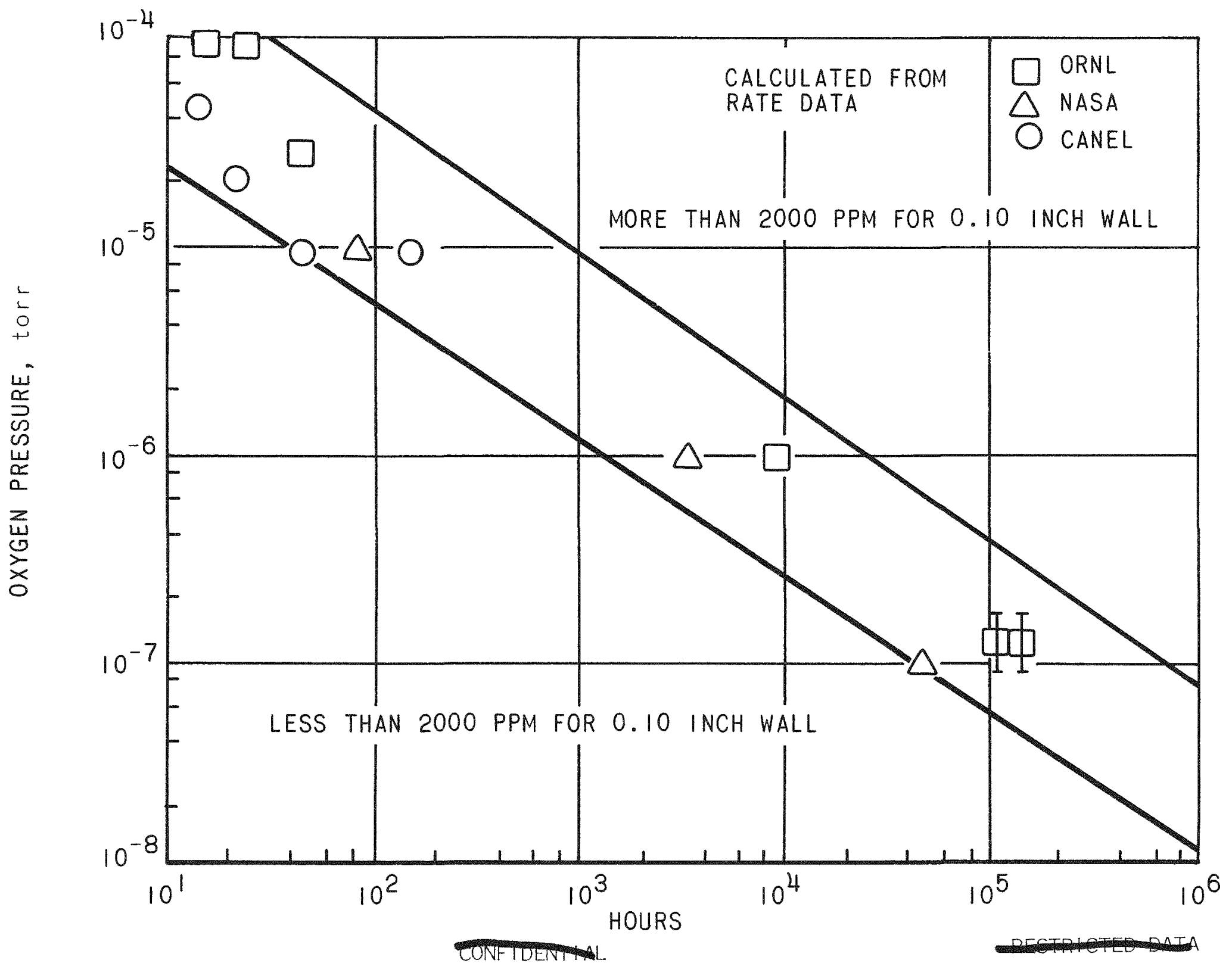

:...::

::.:

(n)

$\therefore: .:$

$\because \because$

$\because \because$

$\because::$

......

$\therefore . .$.

:....: :an: 
PRELIMINARY RESULTS OF CARBON STABILITY TESTS IN VACUUM

POSSIBLE REACTION:

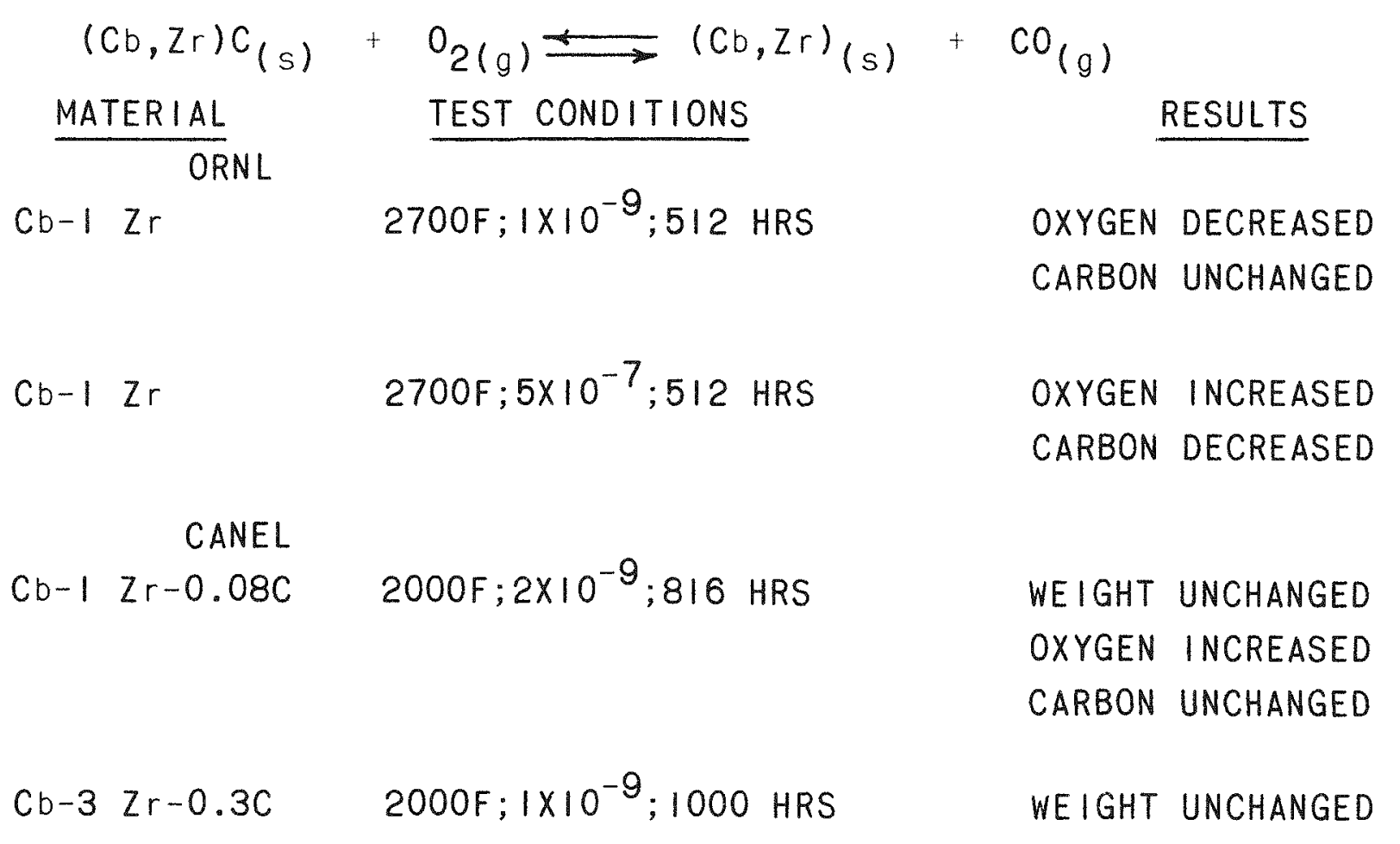

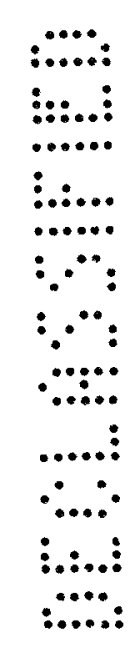




\section{REFLECTOR SUPPORT STRUCTURE}

REQUIREMENTS:

PREHEAT: $\quad 1000 \mathrm{~F}$ AIR/1000 HRS

SERVICE: $\quad 500$ PSI, 1\% CREEP/2400F/10,000 HRS VACUUM

PROGRAM:

STABILITY: SN-AI COATED Cb-I $\mathrm{Zr}$

I000F AIR/1000 HRS/87\% YS

STRENGTH: CREEP AND RUPTURE TEST AFTER PREHEAT

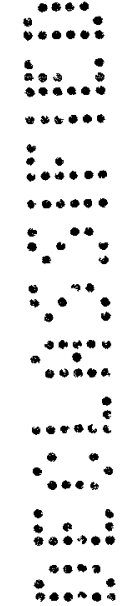


आमणमा

CON

\section{REFLECTOR MATERIAL}

OBJECTIVES:

EFFICIENT, LIGHT WEIGHT

RADIATION STABILITY - $10^{21}$ nvt (.I MEV)

1900F-2400F

$10^{-9}$ torr

MATER IAL:

BEO - SMALL BLOCKS

TENTATIVE IRRADIATION PROGRAM:

EFFECT OF: TEMPERATURE, DOSE, DOSE RATE, DENSITY, GRAIN SIZE

ON: $\quad$ SWELLING,

THERMAL CONDUCTIVITY,

EMISSIVITY

$: \cdots: .:$

$\vdots: \ldots$

(n.......

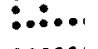

$\because \because$

$\because \because$

$\because:$

- n...

$\therefore \ldots$

$\therefore$

$\therefore: .:$ 


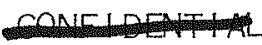

\section{NEUTRON SHIELD REQUIREMENTS}

NEUTRON SHIELD MATERIAL MUST BE:

EFFICIENT (LARGE SCATTERING AND CAPTURE CROSS-SECTIONS)

STABLE (MAINTAIN GEOMETRY AND COMPOSITION, 1000F/1020 nvt)

COMPATIBLE WITH SUPPORT STRUCTURE (1000F/10,000 HRS)

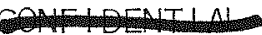

RESTRTET

$\therefore . . .:$

$\vdots: . .:$

(n.....

:.....

$\because \because$

$\because \cdots$

$\because \because$

(.....

$\because \ldots$

$\vdots . .$.

…" 


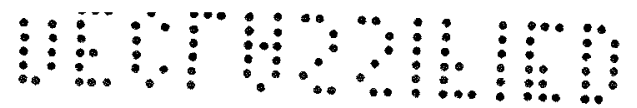

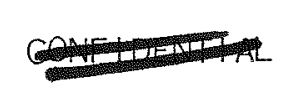

\section{NEUTRON SHIELD MATERIALS}

\begin{tabular}{|c|c|c|c|}
\hline \multirow{2}{*}{\multicolumn{2}{|c|}{$\begin{array}{l}\text { HIGH FLUX REGION } \\
\qquad \mathrm{Li}^{7} \mathrm{H}\left(99.99 \mathrm{~A}^{\circ} \circ \mathrm{Li}^{7}\right)\end{array}$}} & $\sigma / A^{*}$ & \multirow[b]{2}{*}{ MINIMUM HEAT GENERATION } \\
\hline & & 0.25 & \\
\hline & $\mathrm{Be}$ & 0.11 & IMPROVED THERMAL CONDUCTIVITY \\
\hline LOWER & FLUXX REGION & & \\
\hline & LiH (NATURAL Li) & 0.25 & LOWER COST \\
\hline
\end{tabular}

* Removal CROSS Section per nucleon

:...:

$\vdots: . .:$ $\because . .$. (..... $\because \cdots$ $\because \therefore$ $\therefore$ $\because \ldots$$$
\because \ldots
$$$$
\text { :. }
$$

$\therefore .$. 


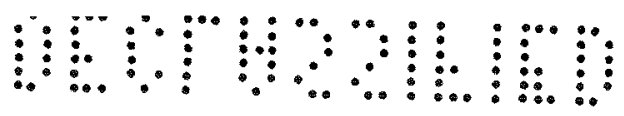

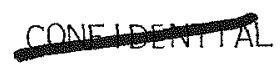

\section{SUMMARY OF LIH TECHNOLOGY}

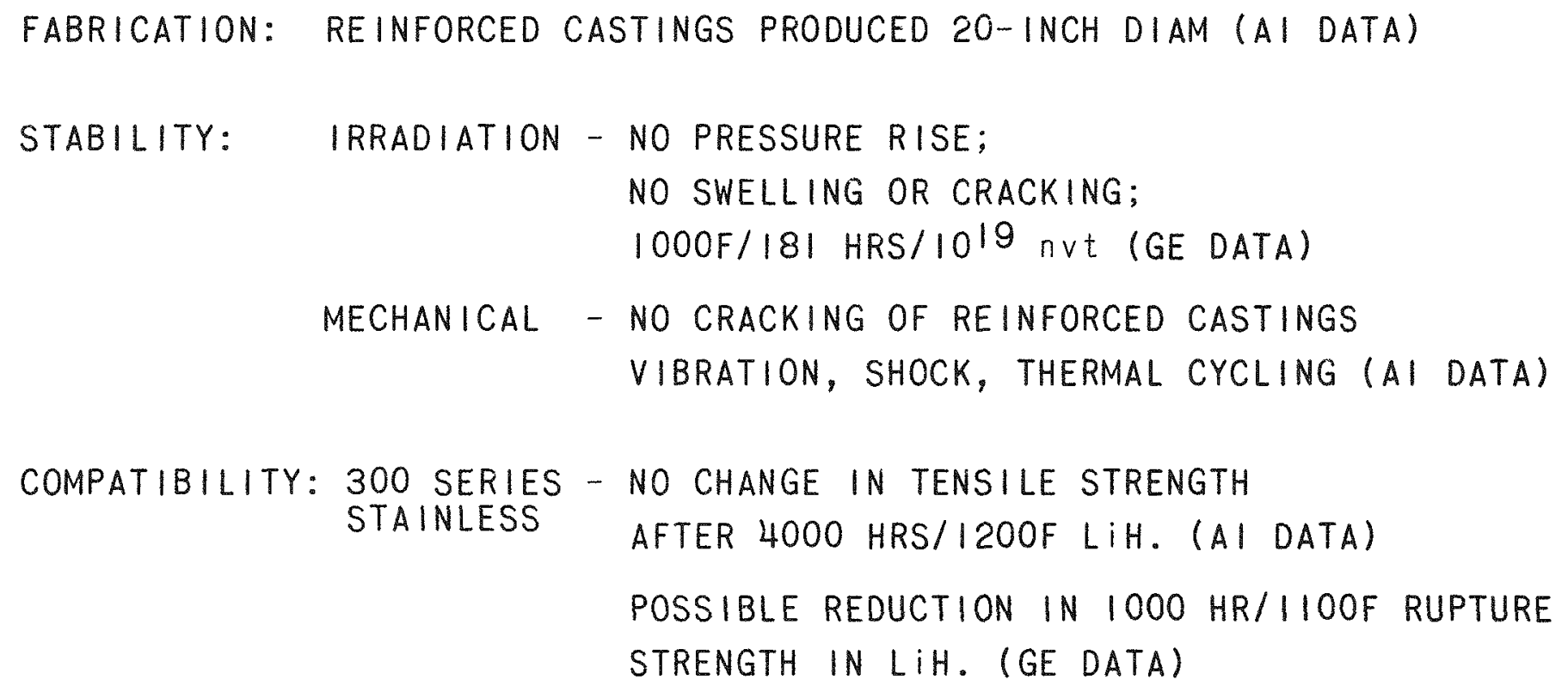




\section{LIH EVALUATION PROGRAM}

DETERMINE:

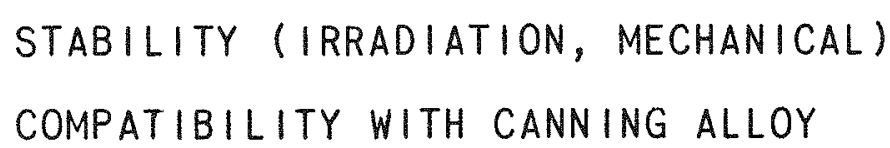

THROUGH:

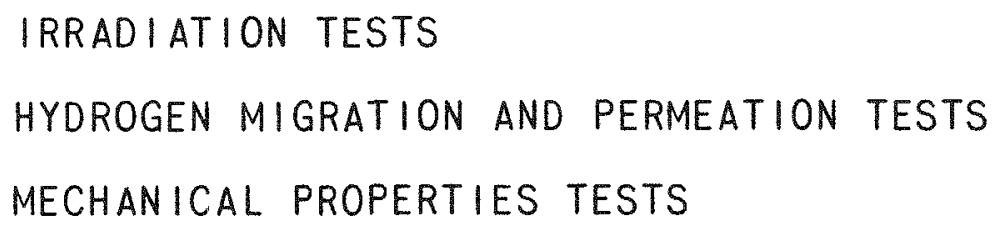

CONDITIONS:

$\begin{array}{ll}\text { IRRADIATION: } & 10 \text { CAPSULES, } 400-1000 \mathrm{~F} \mathrm{Li}{ }^{7} H / 10^{20} \mathrm{nVt} \\ & \text { GAS RELEASE, SWELLING } \\ \text { HYDROGEN: } & 400-1100 \mathrm{~F}, 100-3000 \text { HRS. } \\ \text { MECHANICAL: } & \text { CANNED LIH, } 1100 \mathrm{~F}, \text { PRESSURE } 87 \% \text { YS, TO } 5000 \text { HRS. }\end{array}$


आमआय।

CONF IDEN

CO-LINEAR CONTROL DRIVE, CONTAINED

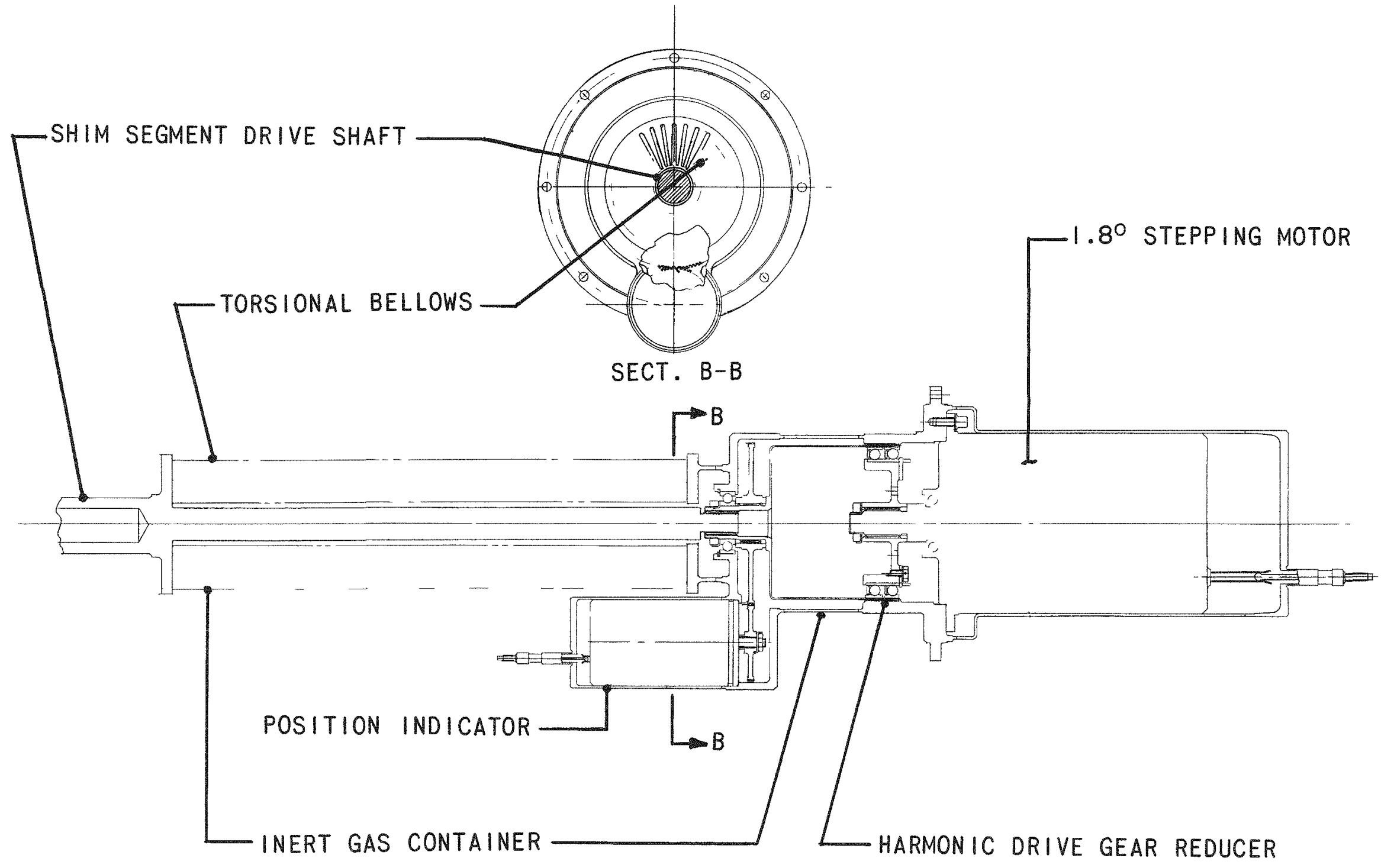

$\therefore: \cdots:$

$\vdots: \ldots$

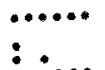

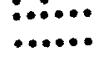

$\because \because$

$\therefore$

$\because \because$

$\ldots \ldots$;

$\therefore$

$\therefore$

$\therefore$ 
आआय

CONELDENT

\section{DUTY CYCLE}

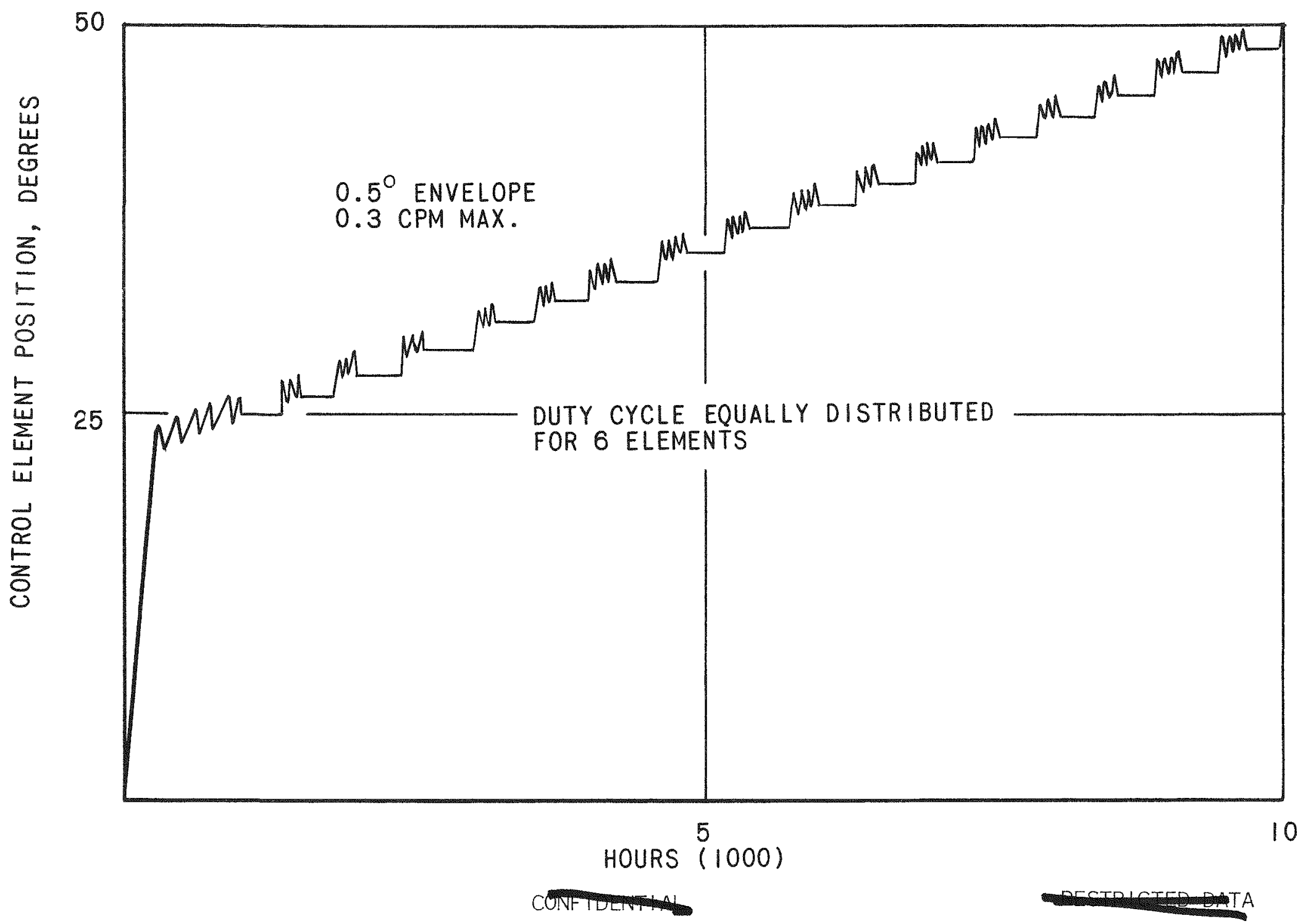

:...:

::..:

$\ldots$

(n):.:

$\because \because$

$\because \because$

$\because \because$

......

$\therefore$

$\therefore$ $\therefore: . .$. 
CONDDALILAT

HIGH TEMPERATURE CONTROL COMPONENT EXPERIENCE

\begin{tabular}{|c|c|c|c|}
\hline COMPONENT & TESTS & HOURS & CONDITION (F) \\
\hline ANTI-FRICTION BEARINGS & 62 & 59,000 & 600 \\
\hline & 6 & 5,000 & 800 \\
\hline HARMONIC BEARINGS & 19 & 11,000 & 600 \\
\hline GEARS & 9 & 10,500 & 600 \\
\hline ELECTRICAL COMPONENTS & 21 & 24,000 & $600-(800)$ \\
\hline
\end{tabular}




\section{CONTROL DRIVE COMPONENT PERFORMANCE OBJECTIVES}

\author{
CONTROL UNIT \\ A. MOTORS \\ B. BEARINGS \\ C. POSITION TRANSDUCERS \\ D. GEARING
}

FLEXURE PIVOTS

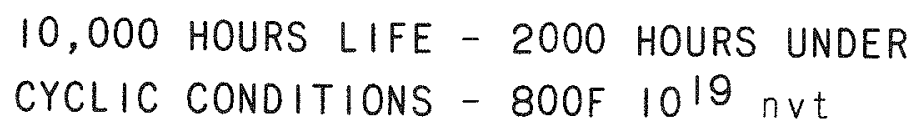

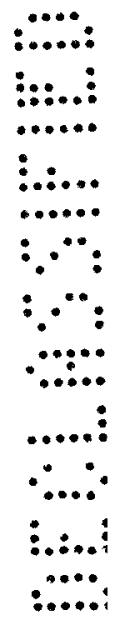




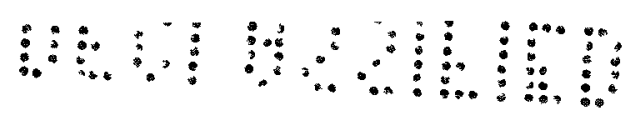

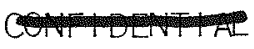

SUMMARY OF SNAP-50 RANKINE CYCLE BOILER DEVELOPMENT AT CANEL

PRIOR TO THE SNAP-50 PROJECT, CANEL HAD BEEN ENGAGED IN THE DEVELOPMENT OF LIGHTWEIGHT, HIGH PERFORMANCE, RELIABLE HEAT EXCHANGERS FOR VARIOUS HIGH TEMPERATURE LIQUID METAL REACTOR SYSTEMS. A BASIC HEAT EXCHANGER CONCEPT EVOLVED FROM THIS WORK.

$$
\begin{aligned}
& \therefore \\
& \text { :::... } \\
& \text { :..... } \\
& \text { ¿...... } \\
& \therefore \cdots \\
& \because \because * \\
& \because \\
& \text {...... } \\
& \therefore \therefore \\
& \text { a.... } \\
& \therefore \text { :...: }
\end{aligned}
$$




\section{HEAT EXCHANGER}

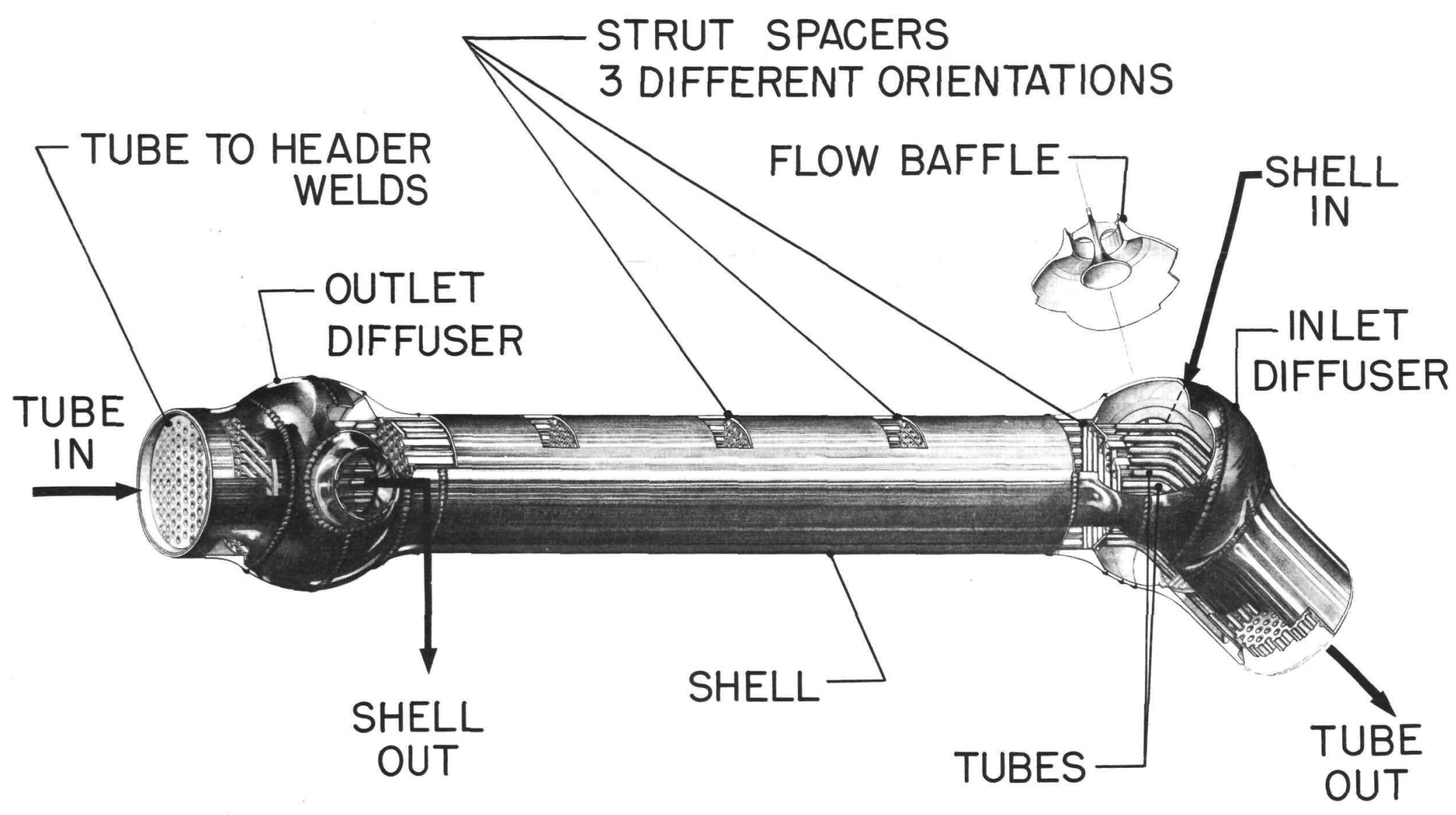


(4)

THATH

MANY HOURS OF DESIGN, COMPONENT AND FULL-SCALE TESTING, SUPPORT THIS CONCEPT. THE NON-NUCLEAR SYSTEMS TEST NOW RUNNING, WHICH HAS BEEN OPERATED FOR MORE THAN 6300 HOURS IN LITHIUM AT 5 MW - 2000F REPRESENTS THE COMBINED RESULTS OF THIS EFFORT. 

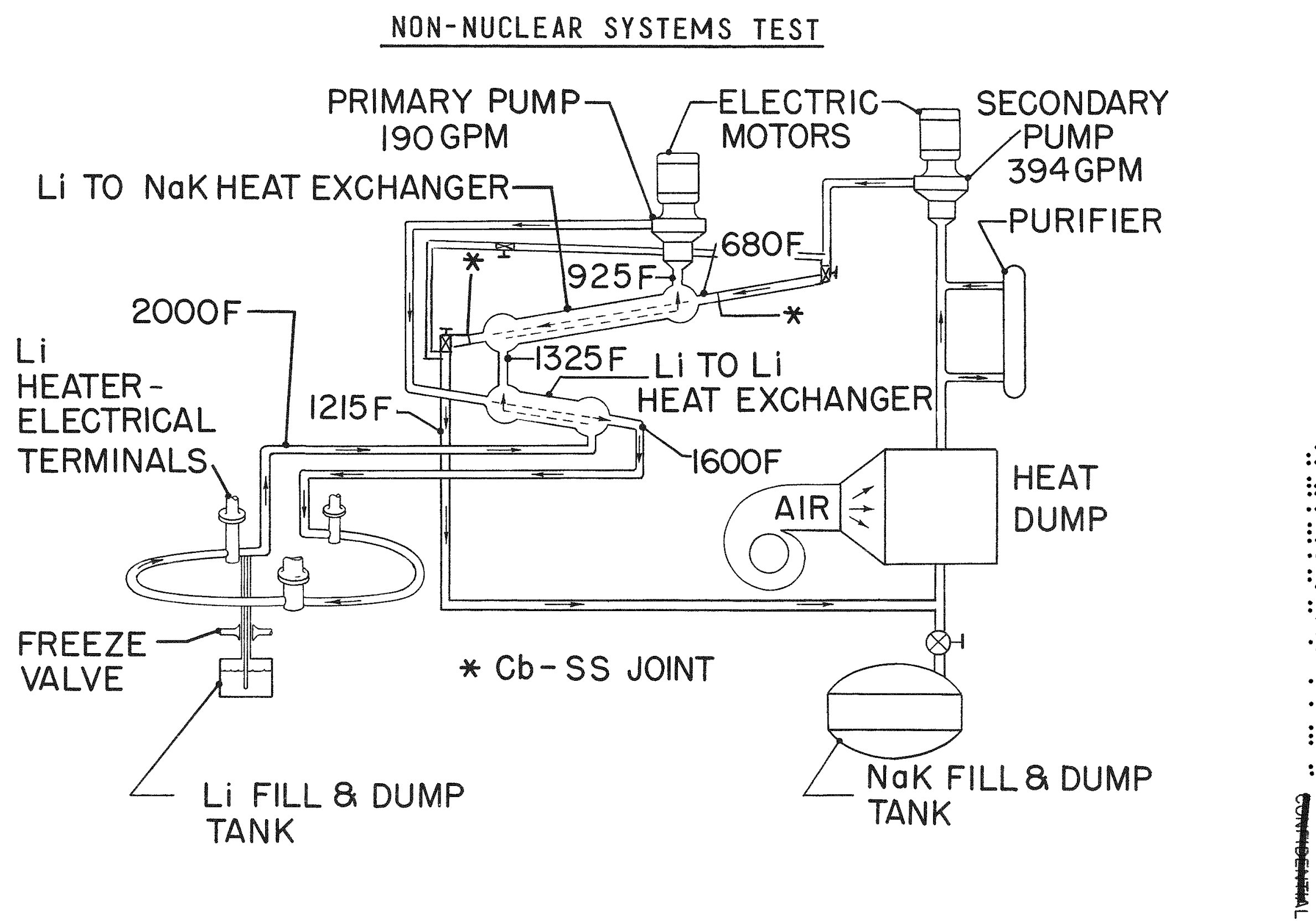


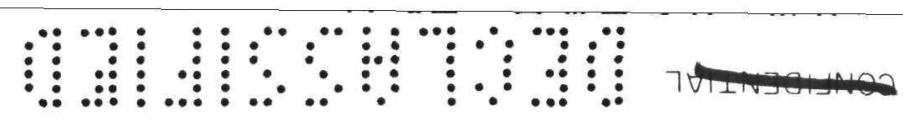

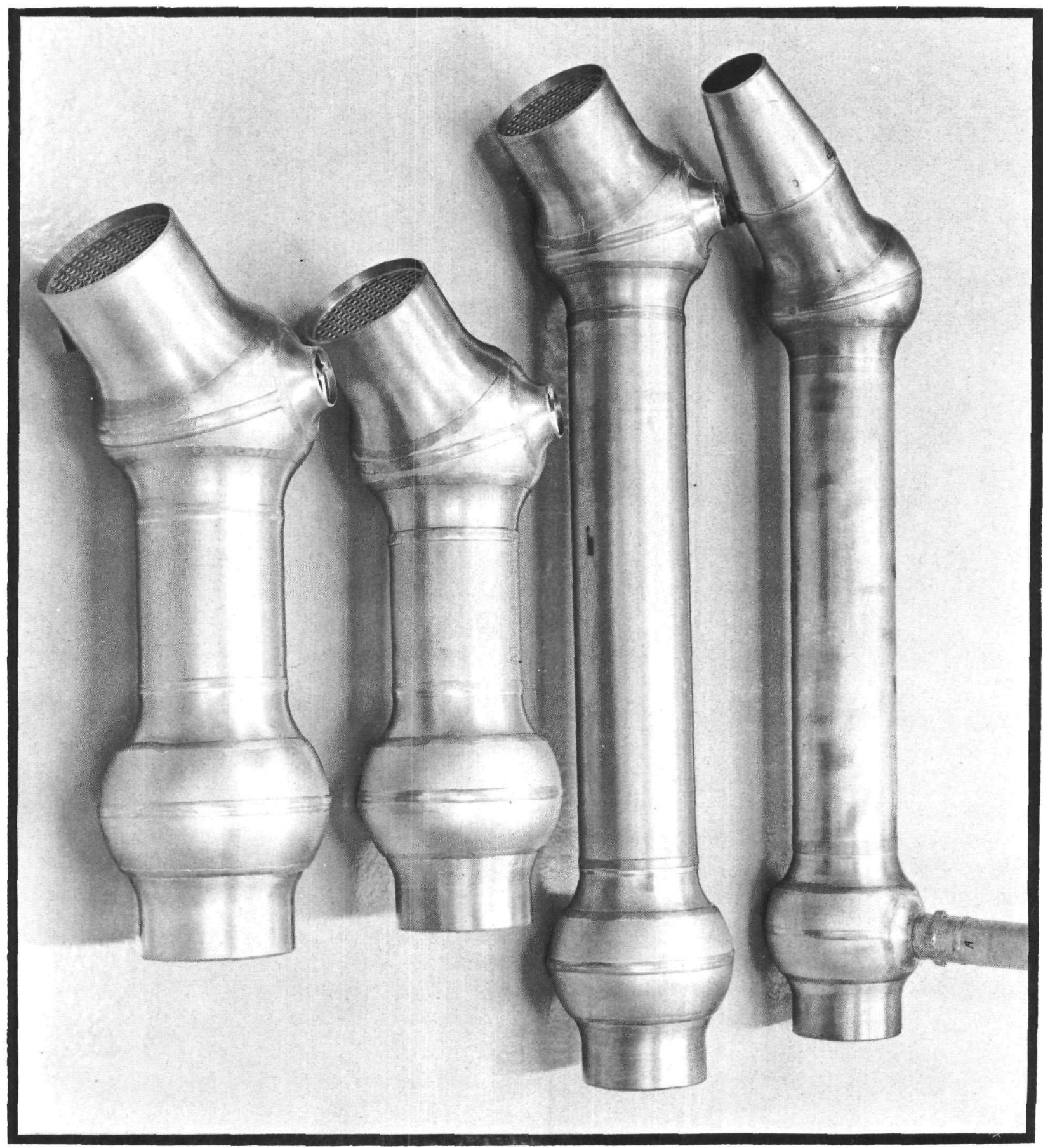

7 VIIVSUT: 
आ

CON WILL

ANALYSIS OF THE SNAP-50 BOILER REQUIREMENTS INDICATED THAT THE BOILER PROBLEM WAS BASICALLY THE SAME AS THE HEAT EXCHANGER PROBLEM WITH THE ADDED REQUIREMENT OF TWO-PHASE ZERO-GRAVITY OPERATION. TWO-PHASE OPERATION INDICATED TWO PROBLEM AREAS (1) DEVELOPMENT OF A ZERO-GRAVITY HEAT TRANSFER MATRIX (BOILER TUBE) AND (2) DEVELOPMENT OF TECHNOLOGY REQUIRED TO DESIGN AND OPERATE A ONCE-THROUGH 100 PERCENT VAPOR QUALITY TWO-PHASE SYSTEM. THE APPROACH TO THE TUBE PROBLEM WAS AS FOLLOWS. 
(4)

CONIAL

\section{BOILER TUBE SELECTION PROGRAM}

\begin{tabular}{llll} 
HEAT TRANSFER 1,2,3 & PRESSURE DROP $1,2,3,4$ & FABRICATION 4 & PACKAGING 4 \\
\cline { 2 - 3 } TEMPERATURE DIFFERENCE & SINGLE PHASE & QUALITY & DIMENSIONAL CONTROL \\
FLOW RATE & TWO PHASE & INSPECTION & SUPPORT \\
PRESSURE & & COST & VOIDS \\
QUALITY & & TIME & STRUCTURAL \\
BOILING INITIATION & & DEVELOPMENT &
\end{tabular}

BOILING STABILITY

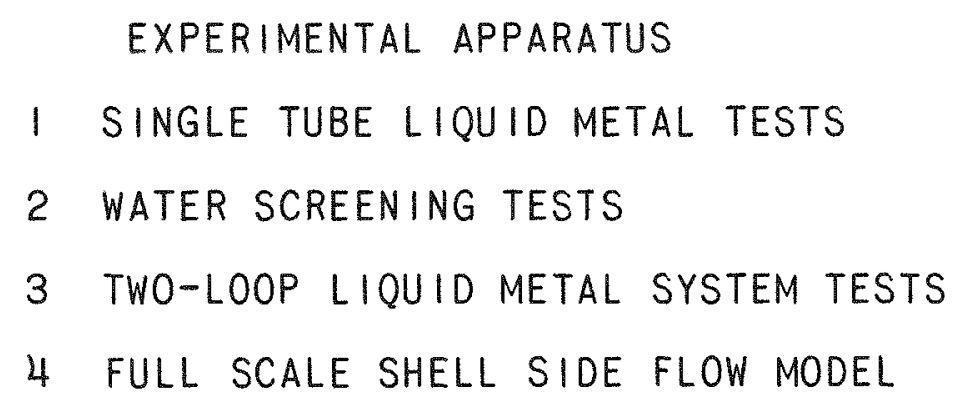

$\ldots: \cdots:$

$\vdots: . . .:$

$\ldots$

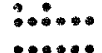

$\because \because$

$\because \cdots$

$\because \cdots$

$\ldots$

$\therefore .$.

$\vdots \ldots$

$\therefore \cdots:$ 


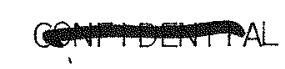

TWO-PHASE POTASSIUM, TUBE SIDE EXPERIMENTAL HEAT TRANSFER AND PRESSURE DROP DATA WERE OBTAINED AS A FUNCTION OF VAPOR QUALITY FOR PARAMETERS OF INTEREST. 
ל:

CONWENTIAL

FORCED CONVECTION LOCAL BOILING HEAT TRANSFER COEFI UILN: VERSUS LOCAL QUALITY - TWO-PHASE POTASSIUM

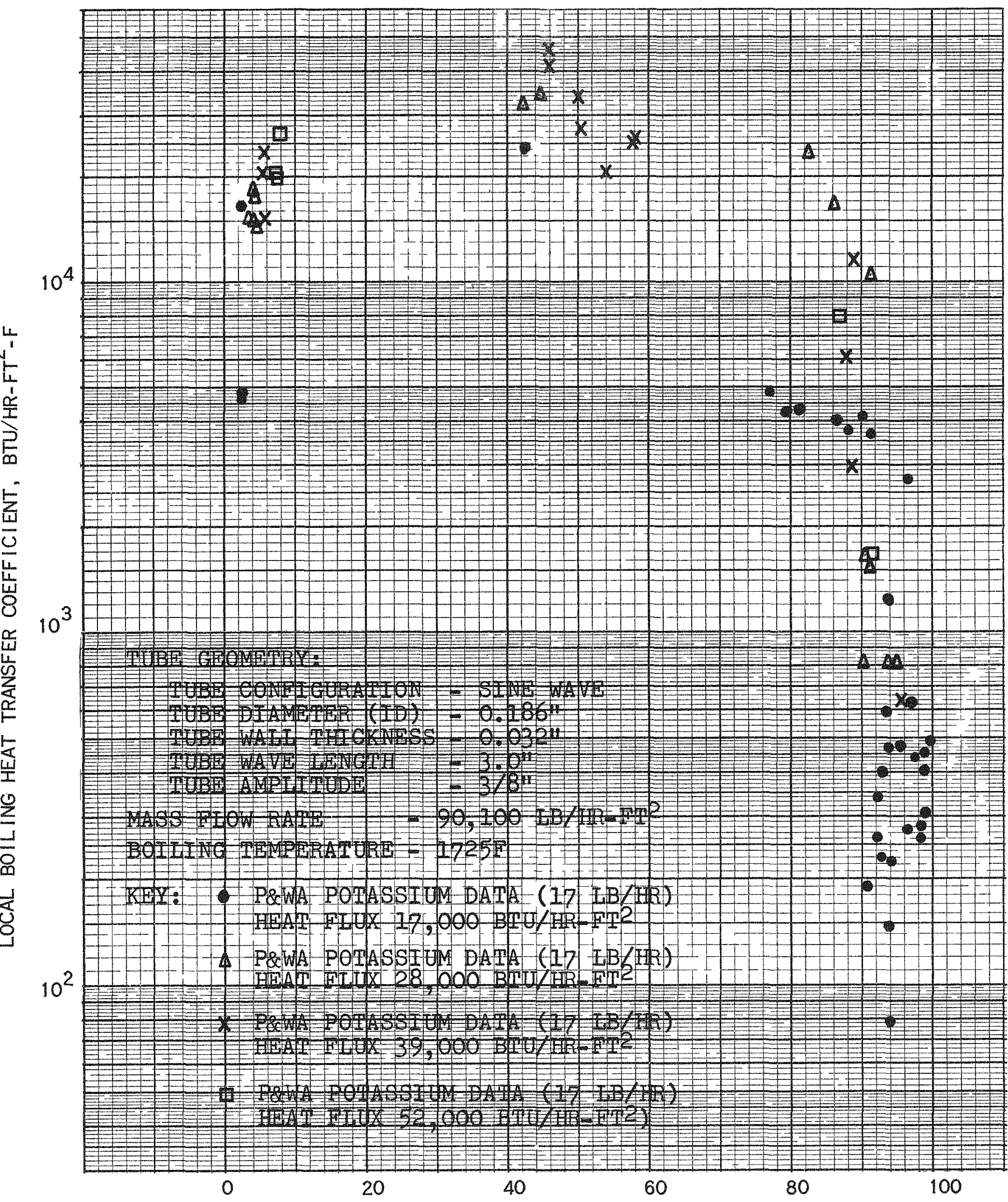


COMPARISON OF EXPERIMENTAL FORCED CONVECTION

LOCAL BOILING PRESSURE COEFFICIENT WITH MARTINELLI-LOCKHART PRESSURE DROP CORRELATION

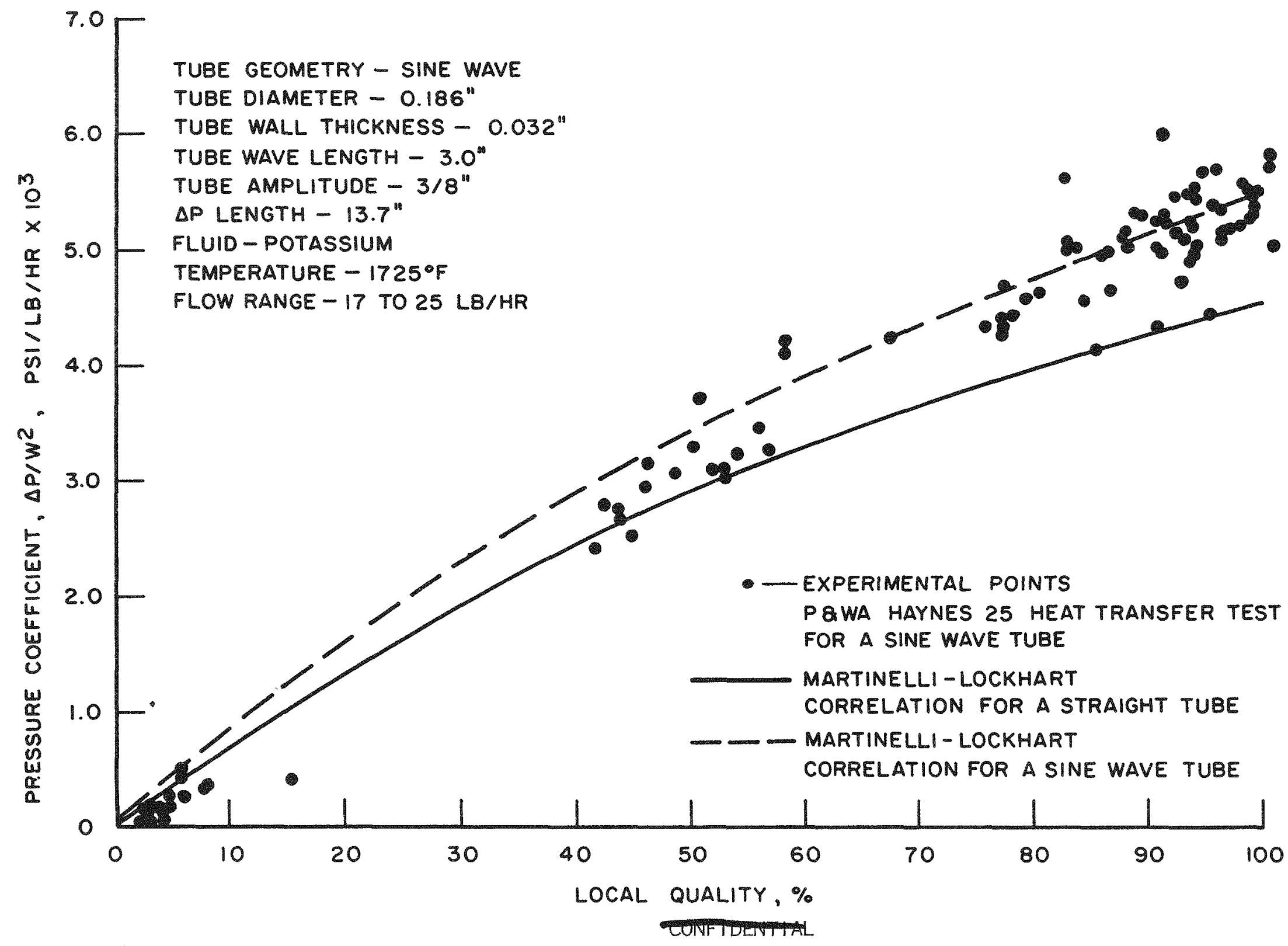

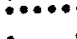

$\vdots: \ldots$

$\ldots$

$\because$

$\therefore$

$\because \because$

$\because \cdots$

(.:...

$\therefore . .$. 


\section{HAYNES 25 BOILING POTASSIUM HEAT TRANSFER RIG}

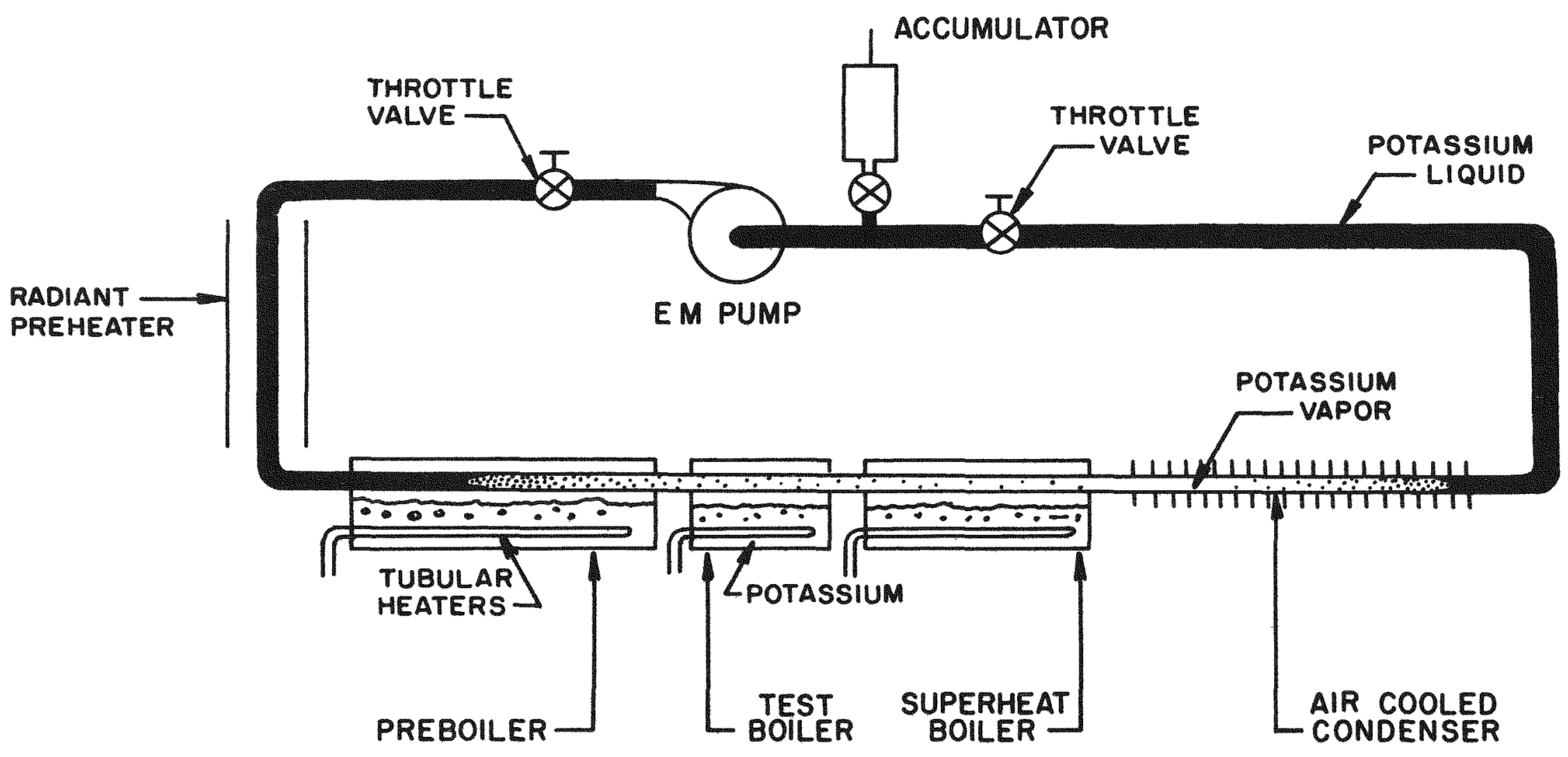

$\therefore: \cdots:$

: :...:

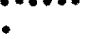

$\vdots \ldots$

$\because$

$\because \cdots$

$\because \cdots$

(.....

$\therefore \therefore$

$\vdots . .$.

$\therefore . . .:$ 
CONEIDEATAL

OVER-ALL TWO-PHASE POTASSIUM HEAT TRANSFER AND PRESSURE DROP DATA WERE OBTAINED ON THREE FULL LENGTH TUBE SHAPES.

$\therefore . . .:$

:::..:

$\because \cdots$

$\vdots . .$.

$\because \because$

$\because \because$

$\because \ldots$

:

$\because$

$\vdots . ., \vdots$

$\therefore . . .:$ 


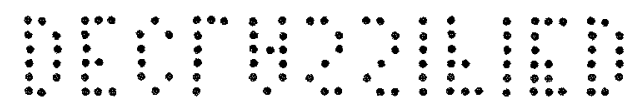

CONCLOATAL

OVERALL HEAT TRANSFER COEFFICIENT, U FOR THE POTASSIUM PREBOILER IN THE P\&WA BOILING POTASSIUM HEAT TRANSFER TEST

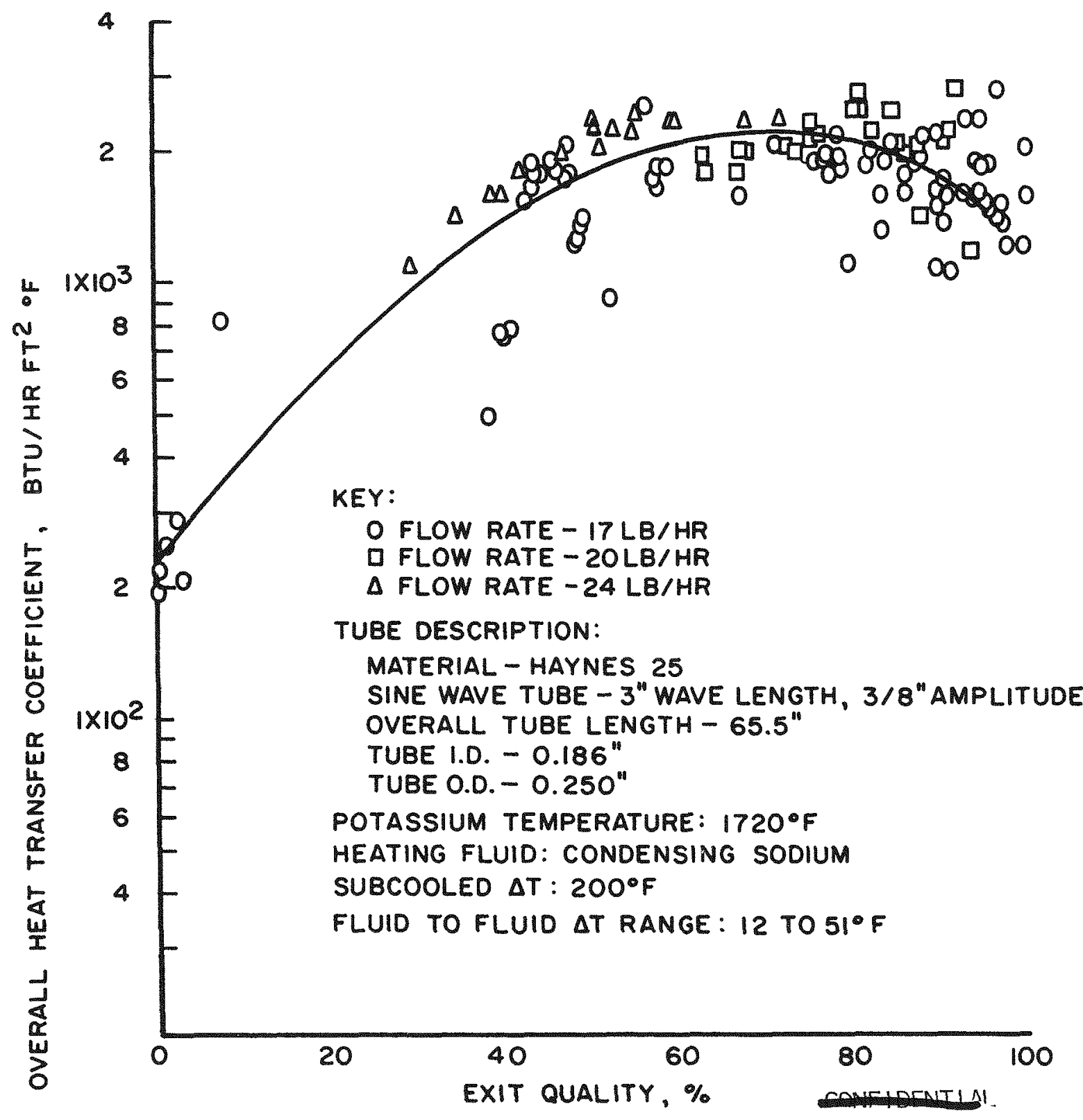


mentenm

CONEIDENTAL

MANY TUBE SHAPES OF INTEREST WERE FABRICATED AND TESTED IN WATER TO DETERMINE THEIR RELATIVE HEAT TRANSFER AND PRESSURE DROP PERFORMANCE. 


\section{BOILER TUBE SAMPLES}

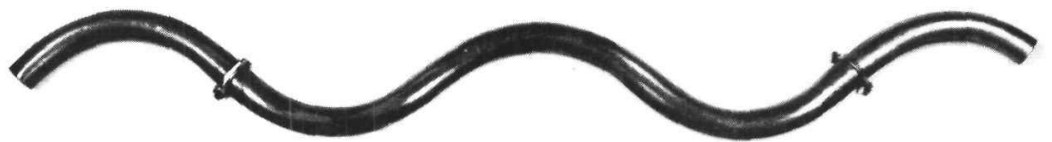

SINE WAVE TUBE

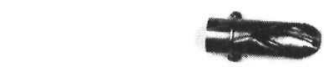

INTEGRAL HELICAL

FIN TUBE

PERFORATED TWISTED

RIBBON TUBE

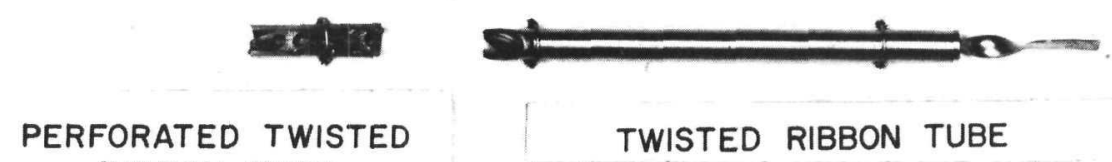

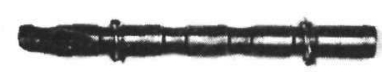

DUAL DIAMETER TUBE

TWISTED RIBBON TUBE

.....

$\therefore \therefore$

....:

-

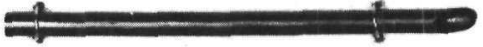

0.127 INCH I.D. TUBE

HELICAL INSERT TUBE

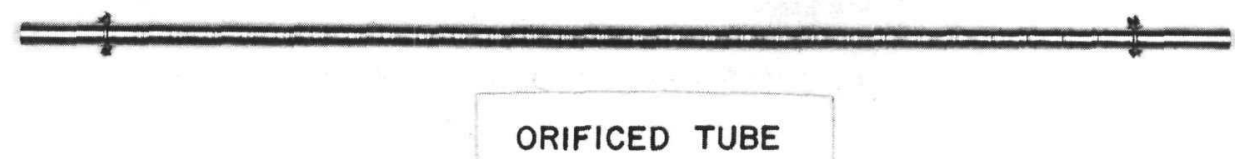

ORIFICED TUBE 
COMPARISON OF LOCAL HEAT TRANSFER COEFFICIENTS FOR VARIOUS BOILER TUBE GEOMETRIES WITHIN A GIVEN PRESSURE DROP RANGE

$\triangle P$ IO-20 INCHES OF WATER AT IOO STEAM QUALITY

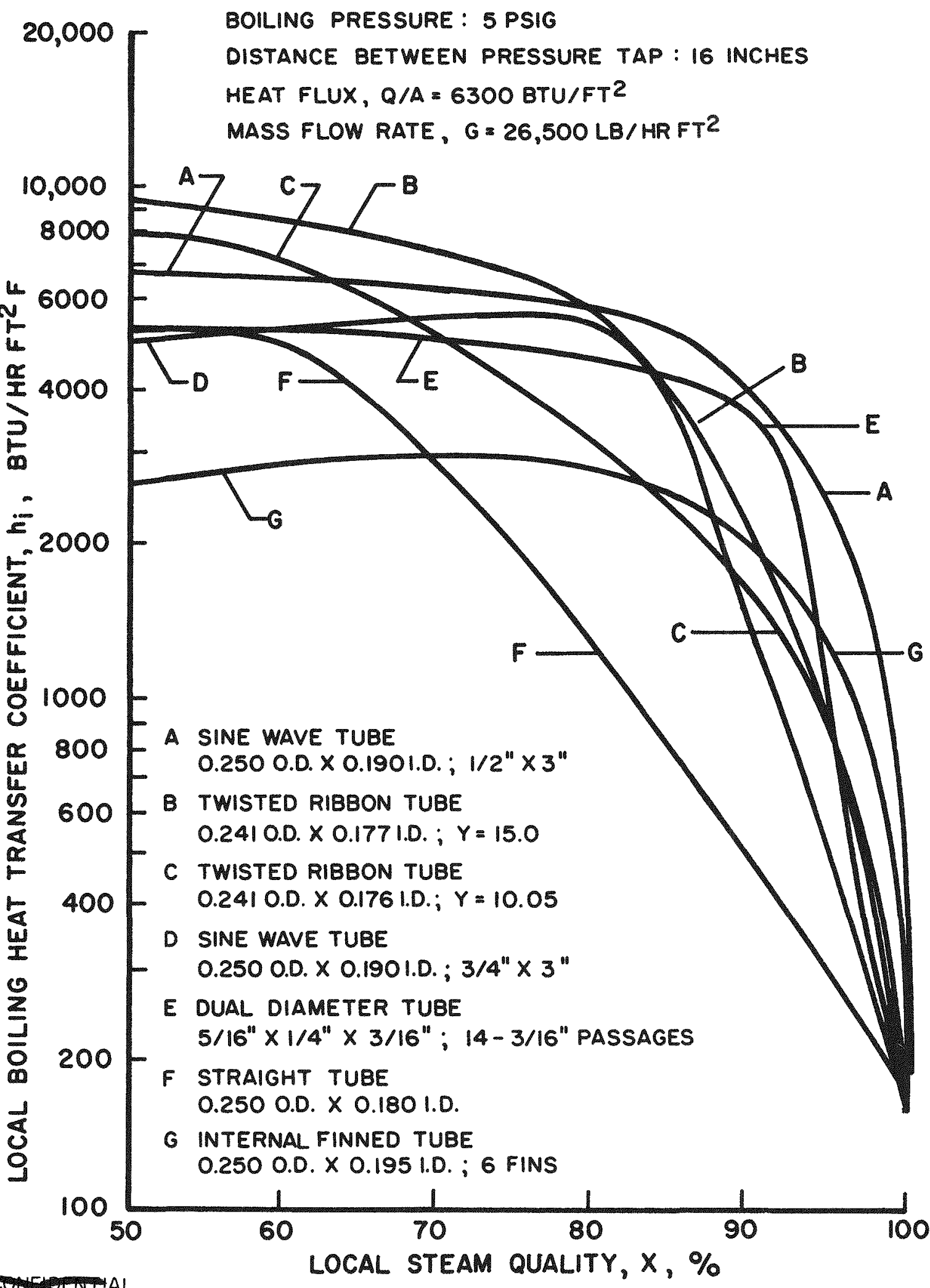




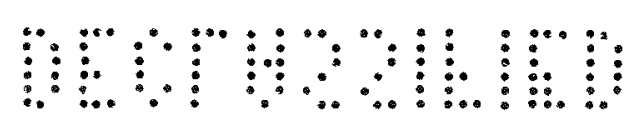

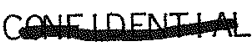

A FULL-SCALE BOILER MODEL INCORPORATING THE SERPENTINE TUBE SIZED IN ACCORDANCE WITH TEST DATA WAS MADE TO DEMONSTRATE FABRICATION AND PACKAGING TECHNIQUES. SHELL SIDE FLOW TESTS TO DETERMINE DISTRIBUTION AND PRESSURE DROP WERE PLANNED FOR THIS MODEL.

$\therefore: \cdots:$

$:: . .: \vdots$

(......

$\therefore \ldots$

$\because \cdots$

$\therefore$

:.:.

.....:

$\therefore \therefore$

$\vdots$ ¿...:

$\therefore . .$. 


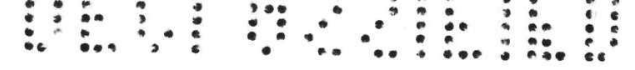

FULL-SIZE SERPENTINE TUBE BOILER MODEL

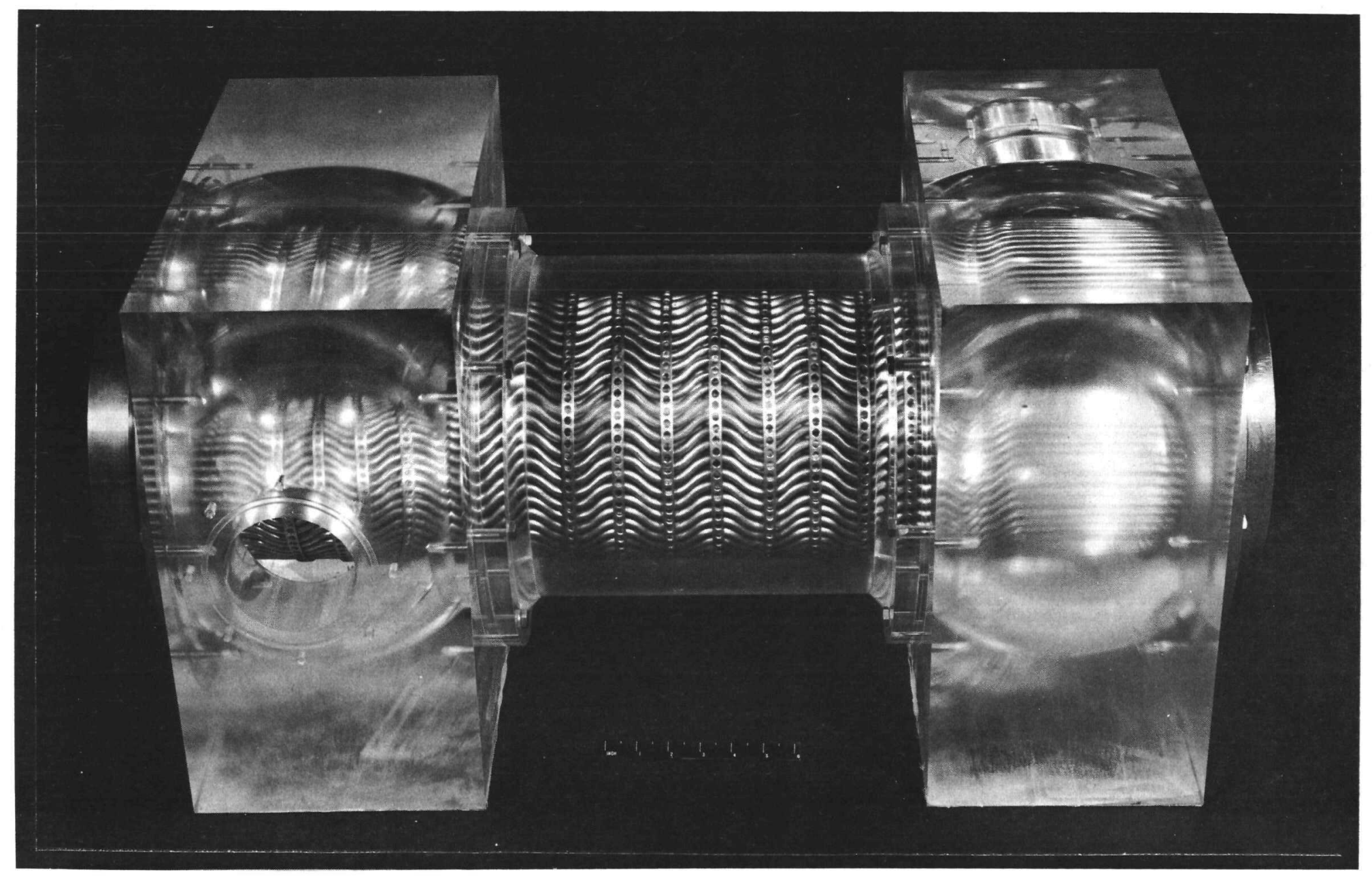

(n)

$: \ldots$

$\ldots$

(n.......

$\because \because$

$\because \because$

$\because \because:$

.....

$\therefore \therefore$

:.:...

$\therefore . .$. 


\section{SINE WAVE BOILER ASSEMBLY}

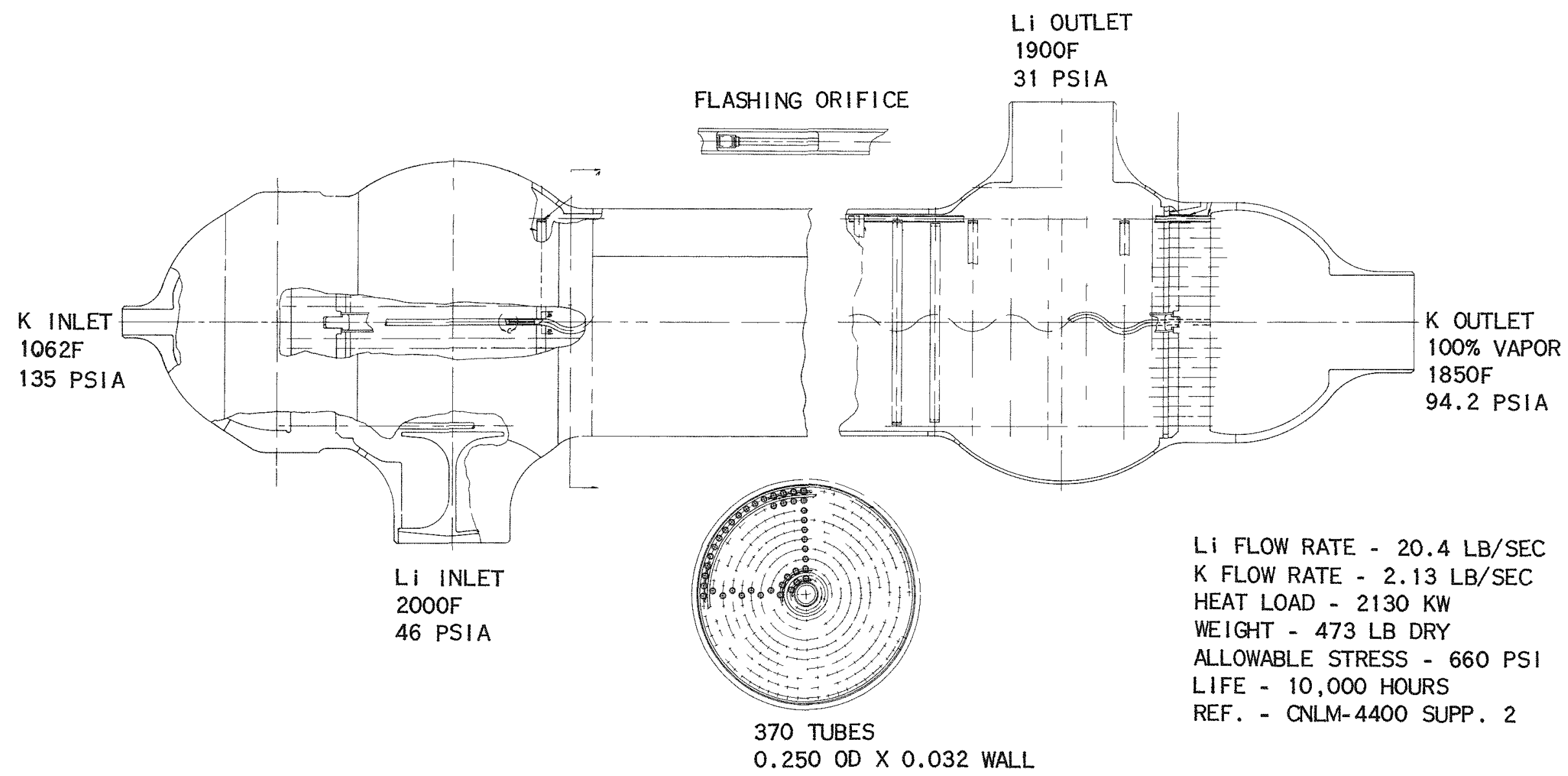

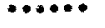
$\because \because$ $\because \because$ $\because \because:$ (......? $\therefore \therefore$ $\vdots . .$. $\therefore: . .:$ 
mentum

CQNLIDENTH

THE TWO-PHASE SYSTEM OPERATIONAL PROBLEM WAS INVESTIGATED IN CONJUNCTION WITH THE HEAT TRANSFER AND PRESSURE DROP TESTS ON THE TWO-LOOP, FULLLENGTH TUBE SYSTEMS.

$\therefore: \cdots:$

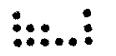

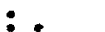

$\therefore$

$\because \because$

$\because \because$

$\because$

......

$\therefore$.

$\vdots \ldots$ …...: 
:

RANGE OF HEAT TRANSFER DATA TAKEN ON STAINLESS STEEL BOILING POTASSIUM LOOP NKSS -B

O.3111.D. TUBE 5 FEET LONG WITH HELICAL INSERT

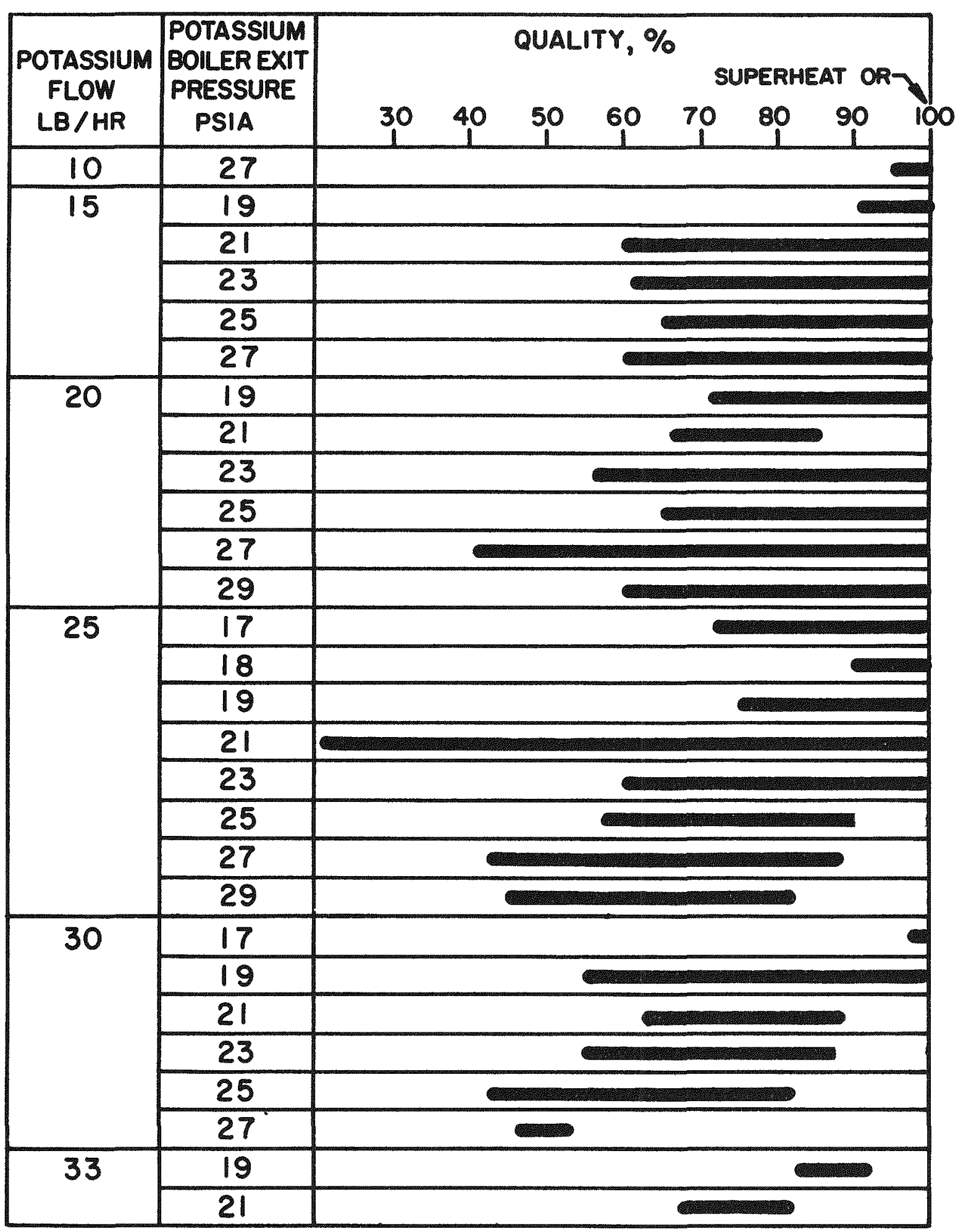


A HAYNES-25, TWO-PHASE POTASSIUM SYSTEM CONTAINING A $70 \mathrm{~kW} 10-$ TUBE BOILER (FULL-SCALE TUBES) AND A MULTI-TUBE CONDENSER IS BEING FABRICATED TO INVESTIGATE SYSTEM AND COMPONENT PROBLEMS OVER A WIDE RANGE OF OPERATING PARAMETERS.

$\therefore: . .:$

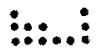

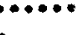

$\therefore$

$\because \because$

$\because \because$

$\ldots$

......

$\therefore .$.

$\vdots$

::.:.:: 
TWO-PHASE POTASSIUM SYSTEM $\quad x-585$

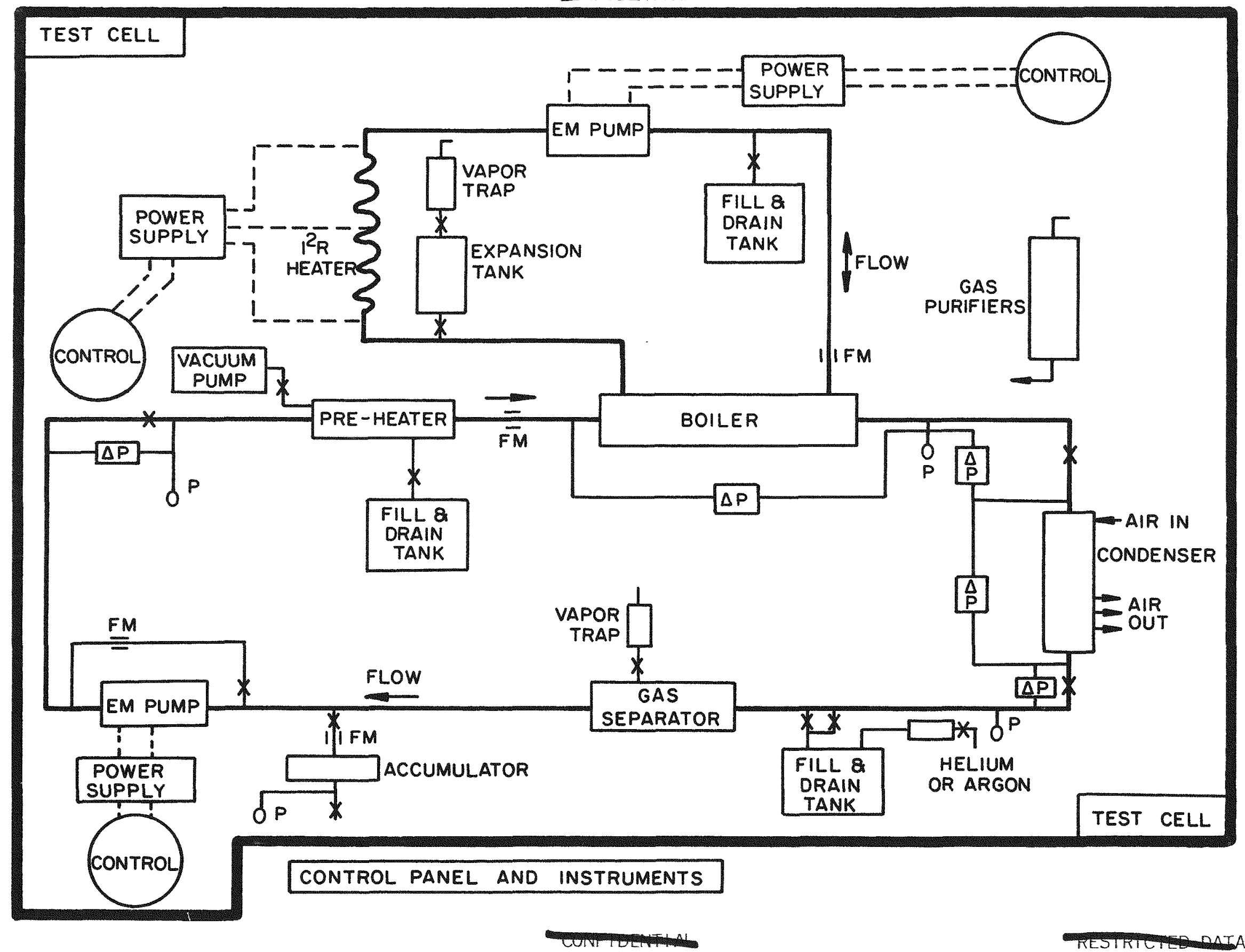

$\therefore \cdots:$

$\vdots: . .$.

- no...

:.....

(n......

$\because$

(n)...

.

$\because$

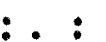

:.:.: 


\section{AREAS OF INVESTIGATION \\ TWO-PHASE POTASSIUM $70 \mathrm{KW}$ SYSTEM TEST}

1. STARTUP CHARACTERISTICS OF A HIGH TEMPERATURE LIQUID METAL MULTI-TUBE TWO-PHASE SYSTEM. (CONTINUATION OF INVESTIGATIONS STARTED UNDER CONTRACT NAS3-2335 BY P\&WA EAST HARTFORD.)

2. CONTROL MODES OF A HIGH TEMPERATURE TWO-PHASE SYSTEM AS INFLUENCED BY THE ACCUMULATOR, POTASSIUM THROTTLE VALVE, CONDENSER, AND I $1^{2} R$ HEATER INPUT.

3. THE LIQUID POTASSIUM PREHEAT AND SUPER-HEAT REQUIRED TO INITIATE AND MAINTAIN STABLE OPERATION.

4. THE REQUIREMENTS FOR AND PERFORMANCE OF A GAS SEPARATOR TO REMOVE NONCONDENSIBLES.

5. DEVELOP A PRACTICAL UNDERSTANDING OF A TWO-PHASE BOILING SYSTEM WHICH. WOULD BE USED TO CONSTRUCT AN ELECTRICAL ANALOG OF THIS SYSTEM AND OTHER SPACE TYPE SYSTEMS SUCH AS SNAP-50/SPUR. 


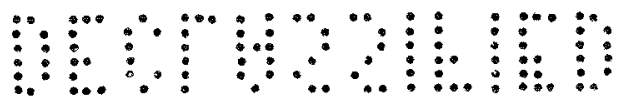

AUXILIARY MATERIALS PROGRAMS

$\frac{\text { COMPONENT }}{\text { PUMP }}$
METAL-CERAMIC JOINT
BEARINGS
SHAFT
$\frac{\text { RADIATOR SYSTEM }}{\text { CONDENSER }}$
RADIATOR
THERMOCOUPLES
CLAD
INSULATION
WIRE

ENV IRONMENT

$1000 \mathrm{~F} \mathrm{Li}$

$O R$

I000F NaK

I250F NaK

I $250 \mathrm{~F} \mathrm{NaK}$

$2200 \mathrm{~F} \mathrm{Li}$

$O R$

$2400 F$ VACUUM

\section{TENTATIVE MATERIALS}

$\mathrm{Cb}-\mathrm{Ir} ; \mathrm{BeO} ; \mathrm{Ti}-8.5 \mathrm{Si}$

WC- 8 Mo-2 CbC

$\mathrm{Mo}-2 \mathrm{OCbC}$

$\mathrm{Cb}-10 \mathrm{~W}-1 \mathrm{Zr}-0.1 \mathrm{C}$

$\mathrm{Cb}-\mathrm{Ir}$

TYPE 316 SS

$\mathrm{Cb}-1 \mathrm{Zr}$

A) $2_{2} \mathrm{O}_{3}$

W-5 Re:W-26 Re $\therefore: .:$ :

$\vdots: . .$.

$\because \ldots$

(a...

$\because \cdots$

$\because \because$

$\because \because:$

$\because: \%$

......

$\therefore .$.

$\vdots: . .$.

:...:: 


\section{PROCESS SENSOR DEVELOPMENT}

SUPPORTS CANEL REQUIREMENTS FOR:

\section{REACTOR TEST}

GENERAL DEVELOPMENT TESTING

ADVANCED MATERIALS PROGRAM

COLUMBIUM FABRICATION

:...:

::...:

$\ldots$ $\because \because$

$\because \cdots$ $\therefore \ldots$

$\ldots$

$\because \ldots$

$\vdots$ $\therefore \cdots:$ 
आआयแ।

CON UENTRL

\section{PROCESS SENSOR DEVELOPMENT}

PRIMARY EFFORT

THERMOCOUPLES

LIQUID METAL LEVEL PROBES

RADIATION DETECTORS
APPLICATION

REACTOR AND PROCESS CONTROL

FUELS IRRADIATION PROGRAM

PROCESS CONTROL

REACTOR CONTROL

CRITICAL EXPERIMENTS

IRRADIATION TESTING $\therefore \ldots:$ :

:::..:

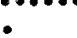

$\therefore . .$.

$-1$

$\therefore$

$\therefore \because$

$\because \because:$

(......

$\therefore \ldots$

$\therefore \ldots$

$\therefore: . .:$ : 


\section{PROCESS SENSOR DEVELOPMENT}

\section{COMPLETED}

\section{TYPE \\ CHROMEL/ALUMEL} W-5 Re/W-26 Re
QUANTITY TEM 20

\section{$\mathrm{Cb}-\mathrm{I} \mathrm{Zr}$ CLAD THERMOCOUPLE TESTS}

TEMPERATURE, $F$ TIM TIME, HR

ENV IRONMENT

POST-TEST ACCURACY

\section{PERCENT}

SURVIVING

1950

10,000

INERT GAS

$1 \%$

$1 \%$

100

100

IN PROGRESS

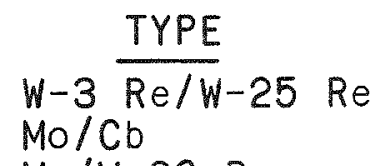

Mo/W-26 Re

\begin{tabular}{c} 
TEMPERATURE, F \\
\hline 1950 \\
1950 \\
1950
\end{tabular}

SCHEDULED

TIME, HR
10,000
10,000
10,000
TIME TO
DATE, HR
4600
2300
9500

$\begin{array}{ll}\frac{\text { ACCURACY }}{1 \%} & \begin{array}{l}\text { PERCENT } \\ \text { SURVIVING }\end{array} \\ \begin{array}{ll}100 \\ \text { NOT DEFINED } & 100 \\ \text { NOT DEFINED } 100 & \end{array}\end{array}$

IN PROCESS OF BUILD-UP

\begin{tabular}{|c|c|c|c|c|}
\hline TYPE & QUANTITY & TEMPERATURE, F & SCHEDULED TIME, HR & ENVIRONMENT \\
\hline $\begin{array}{l}\text { HROMEL/ALUMEL } \\
-5 \mathrm{Re} / W-26 \mathrm{Re} \\
-5 \mathrm{Re} / \mathrm{W}-26 \mathrm{Re} \\
-5 \mathrm{Re} / \mathrm{W}-26 \mathrm{Re} \\
-3 \mathrm{Re} / \mathrm{W}-25 \mathrm{Re} \\
\text { HROMEL/ALUMEL } \\
\text { o/Cb } \\
\text {-5 Re/W-26 Re }\end{array}$ & $\begin{array}{r}6 \\
12 \\
70 \\
100 \\
40 \\
20 \\
20 \\
20\end{array}$ & $\begin{array}{c}2200 \\
2200 \\
2200-2400 \\
2200-2500 \\
2300-2500 \\
2200 \\
2300-2500 \\
2500\end{array}$ & $\begin{array}{c}10,000 \\
10,000 \\
1000-10,000 \\
10,000 \\
10,000 \\
10,000 \\
10,000 \\
10,000\end{array}$ & $\begin{array}{l}\text { NaK } \\
\text { NaK } \\
\text { LITHIUM } \\
\text { INERT GAS } \\
\text { INERT GAS } \\
\text { INERT GAS } \\
\text { INERT GAS } \\
\text { VACUUM }\end{array}$ \\
\hline
\end{tabular}

$\therefore: .:$

::...:

- no...

$\therefore \ldots$

$\because \cdots$

$\because \because$

$\because \cdots$

$\because \because:$

(......

$\therefore \therefore$

¿...:

$\therefore: \cdots:$ 


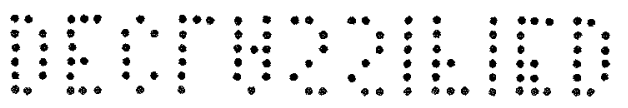

\section{PROCESS SENSOR DEVELOPMENT}

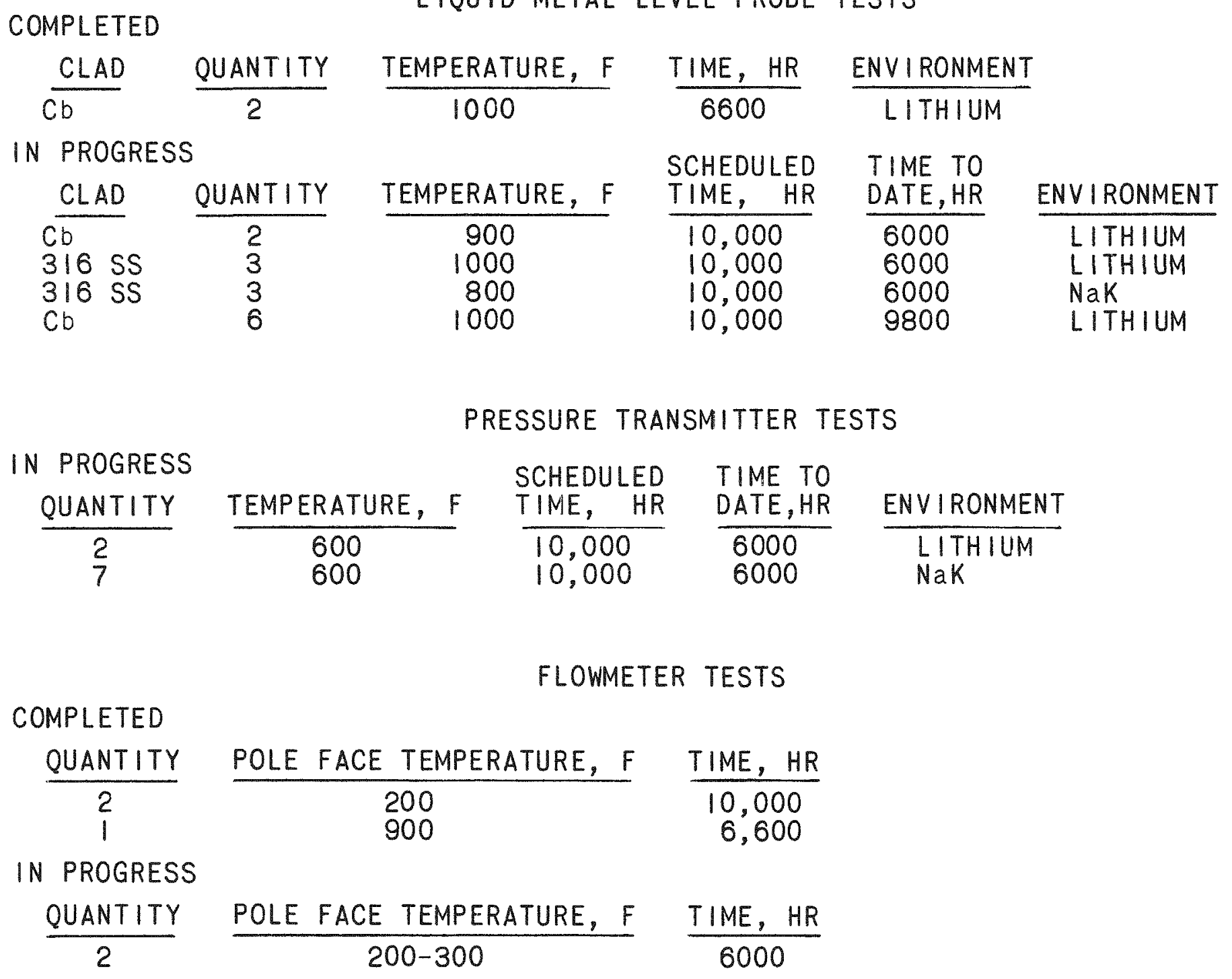




\section{PROCESS SENSOR DEVELOPMENT}

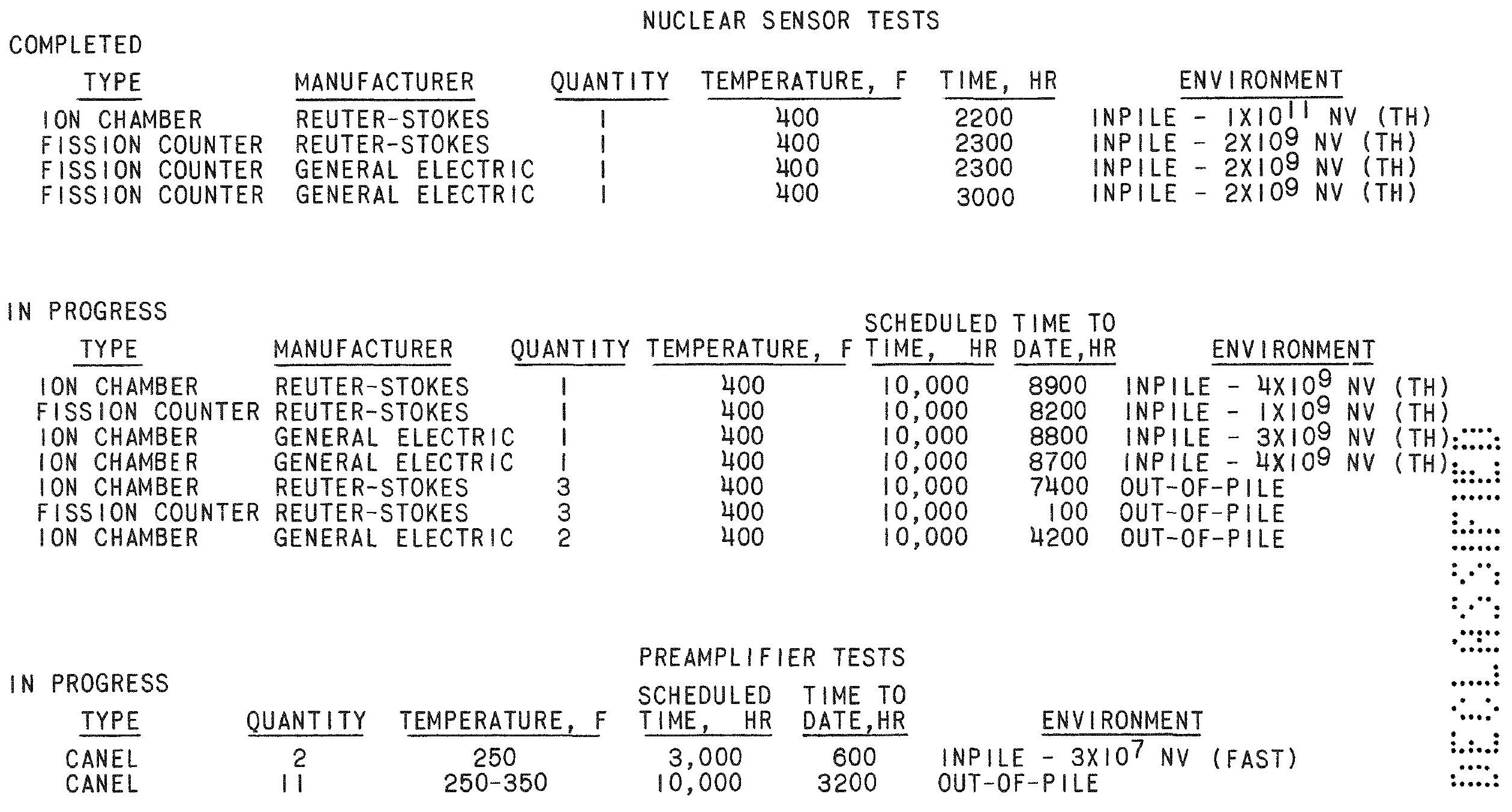




\section{PROCESS SENSOR CAPABILITY}

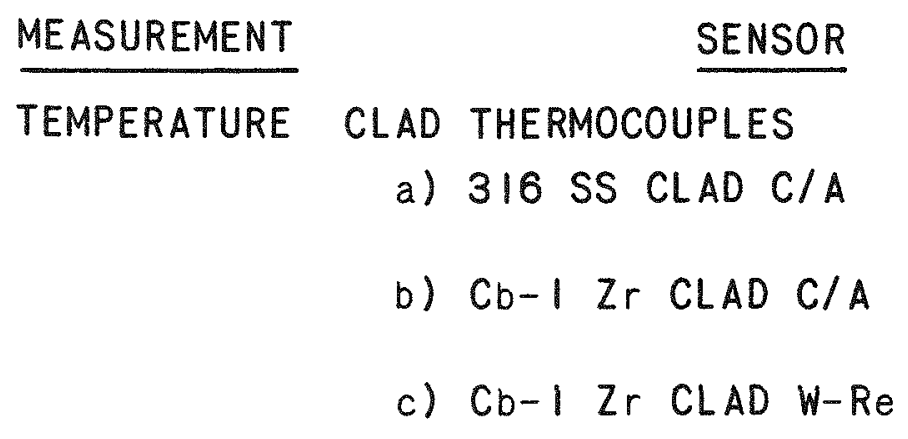

PRESSURE TRANSMITTERS, DIAPHRAGM TYPE, 316 SS

FLOW PERMANENT MAGNETS

RADIATION FISSION AND IONIZATION CHAMBERS

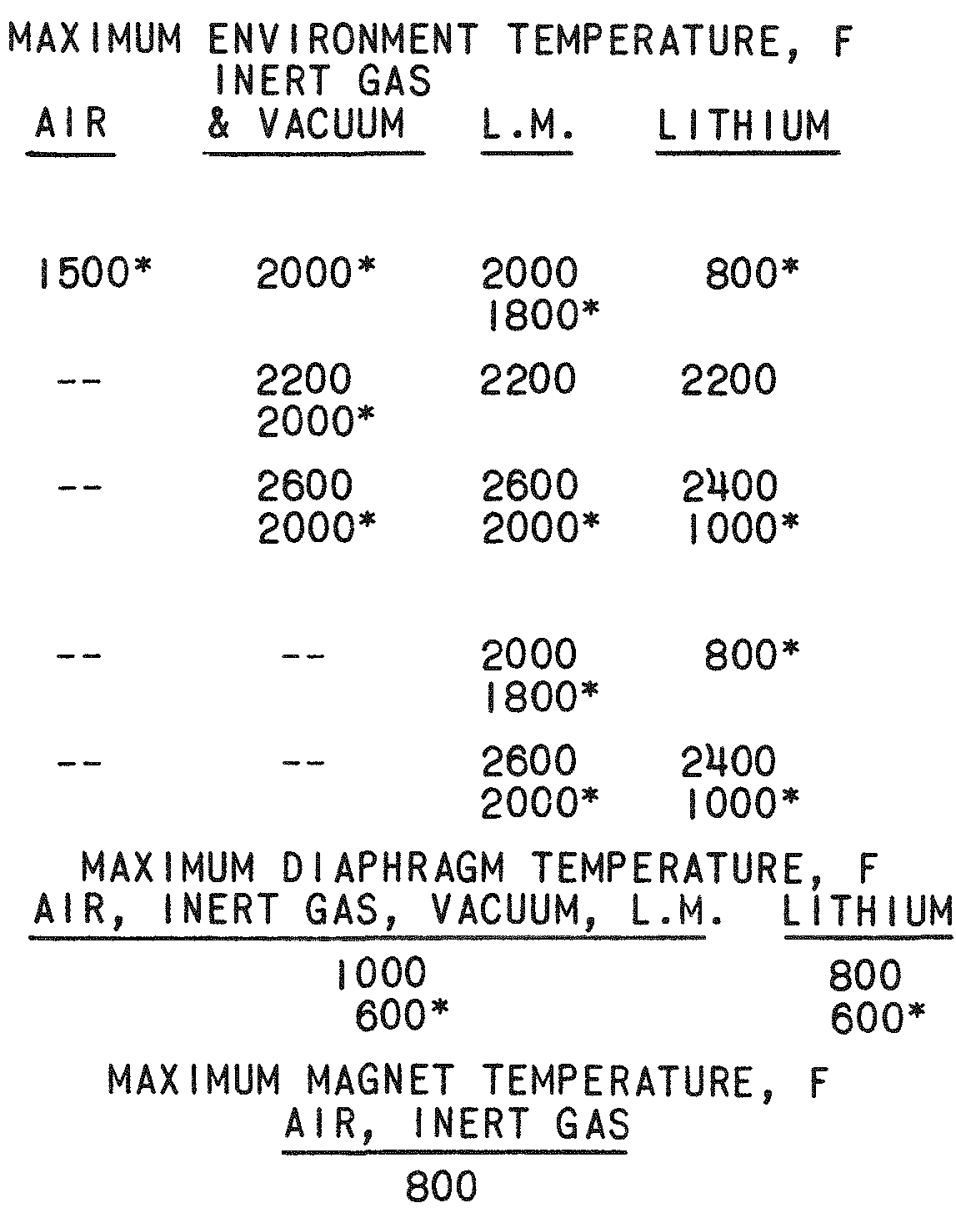

MAXIMUM ENVIRONMENT TEMPERATURE, $F$ $\frac{\text { AIR, INERT GAS }}{700}$ $\therefore:$ : $:: . .:$ (n.... :..... $\therefore \because$<smiles>[13CH3]</smiles>

$\because \cdots$

(n)

(.....:

$\therefore \ldots$

$\vdots \ldots$ $\therefore: .:$ :

*PROVEN LONG TERM PERFORMANCE 
मणओ आ।

SUMMARY OF LIQUID METAL PUMP DESIGN EXPERIENCE AT CANEL

\begin{tabular}{|c|c|c|c|c|c|c|}
\hline ANP PROGRAM & & & & & SHAFT & \\
\hline MODEL & FLUID & $\begin{array}{l}\text { MAX. } \\
\text { TEMP, F }\end{array}$ & $\begin{array}{l}\text { FLOW RATE, } \\
\text { GPM }\end{array}$ & $\begin{array}{l}\text { HEAD RISE, } \\
\text { FT. }\end{array}$ & $\begin{array}{l}\text { SPEED, } \\
\text { RPM }\end{array}$ & TYPE DRIVE \\
\hline$N J-2 B$ & NaK-44 & 1200 & 8500 & 500 & 4350 & AIR TURBINE \\
\hline$T P-1$ & $\mathrm{NaK}-44$ & 1300 & 3000 & 440 & 8000 & AIR TURBINE \\
\hline$P W-19-1$ & $\mathrm{NaK}-44$ & 1500 & 47 & 120 & 4500 & ELECTRIC MOTOR \\
\hline$T P-2$ & $\mathrm{NaK}-44$ & 1342 & 8500 & 540 & 7150 & AIR TURBINE \\
\hline LCRE PROGRAN & & & & & & \\
\hline LP-I & LITHIUM & 1600 & 195 & 115 & 4500 & ELECTRIC MOTOR \\
\hline$N P-1$ & $\mathrm{NaK}-78$ & 1200 & 394 & 143 & 4580 & ELECTRIC MOTOR \\
\hline$R P-1$ & $\mathrm{NaK}-78$ & 700 & 175 & 106 & 4300 & ELECTRIC MOTOR \\
\hline SNAP-50/SPUF & R PROGRAM & & & & & \\
\hline LP-2 & LITHIUM & 1877 & 341 & 130 & 11,400 & ELECTRIC MOTOR \\
\hline$K P-2$ & POTASSIUM & 1103 & 43 & 339 & 11,400 & ELECTRIC MOTOR \\
\hline$N P-2$ & $\mathrm{NaK}-78$ & 1080 & 131 & 60 & 11,400 & ELECTRIC MOTOR \\
\hline
\end{tabular}

$\therefore: \cdots:$

$:: . . .:$

8.

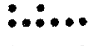

$\because$

$\therefore$

$\because \because$

......

$\therefore$

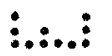

$\therefore:$ 
मआयाओ

TIT

\section{SUMMARY OF LIQUID METAL PUMP DEVELOPMENT EXPERIENCE AT CANEL}

\begin{tabular}{|c|c|c|c|c|c|c|}
\hline MODEL & FLUID & TEMPERATURE, F & $\begin{array}{l}\text { FLOW RATE, } \\
\text { GPM }\end{array}$ & $\begin{array}{l}\text { HEAD RISE, } \\
\text { FT }\end{array}$ & $\begin{array}{l}\text { LONGEST } \\
\text { TEST, HRS } \\
\end{array}$ & $\begin{array}{l}\text { TOTAL } \\
\text { OPERAT ING } \\
\text { HOURS } \\
\end{array}$ \\
\hline$L P-1$ & LITHIUM & $\begin{array}{l}1600 \text { MAX. } \\
1000 \text { NOM. }\end{array}$ & 195 & 140 & $\begin{array}{l}10,292 \\
10,112\end{array}$ & 40,205 \\
\hline$N P-1$ & $\mathrm{NaK}-78$ & $\begin{array}{r}1200 \text { MAX. } \\
700 \text { NOM. }\end{array}$ & 394 & 143 & 8,113 & 19,689 \\
\hline$T P-1$ & $\mathrm{NaK}-44$ & $\begin{array}{l}1300 \text { MAX. } \\
\text { I } 200 \text { NOM. }\end{array}$ & 3000 & 440 & 1, 332 & 4,408 \\
\hline$P W-19-1$ & NaK-44 & 1500 & 47 & 120 & 911 & 2,640 \\
\hline$S-1$ & $\mathrm{NaK}-44$ & $\begin{array}{l}1335 \text { MAX. } \\
1000 \text { NOM. }\end{array}$ & 400 & 127 & 1,000 & 2,326 \\
\hline \multirow[t]{2}{*}{$s-2$} & $\mathrm{NaK}-44$ & 1200 & 400 & 127 & 70 & 70 \\
\hline & & & & & TOTAL & 69,338 \\
\hline
\end{tabular}

MAY 8, 1964

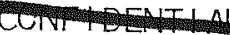




\section{PUMP}

आLoy

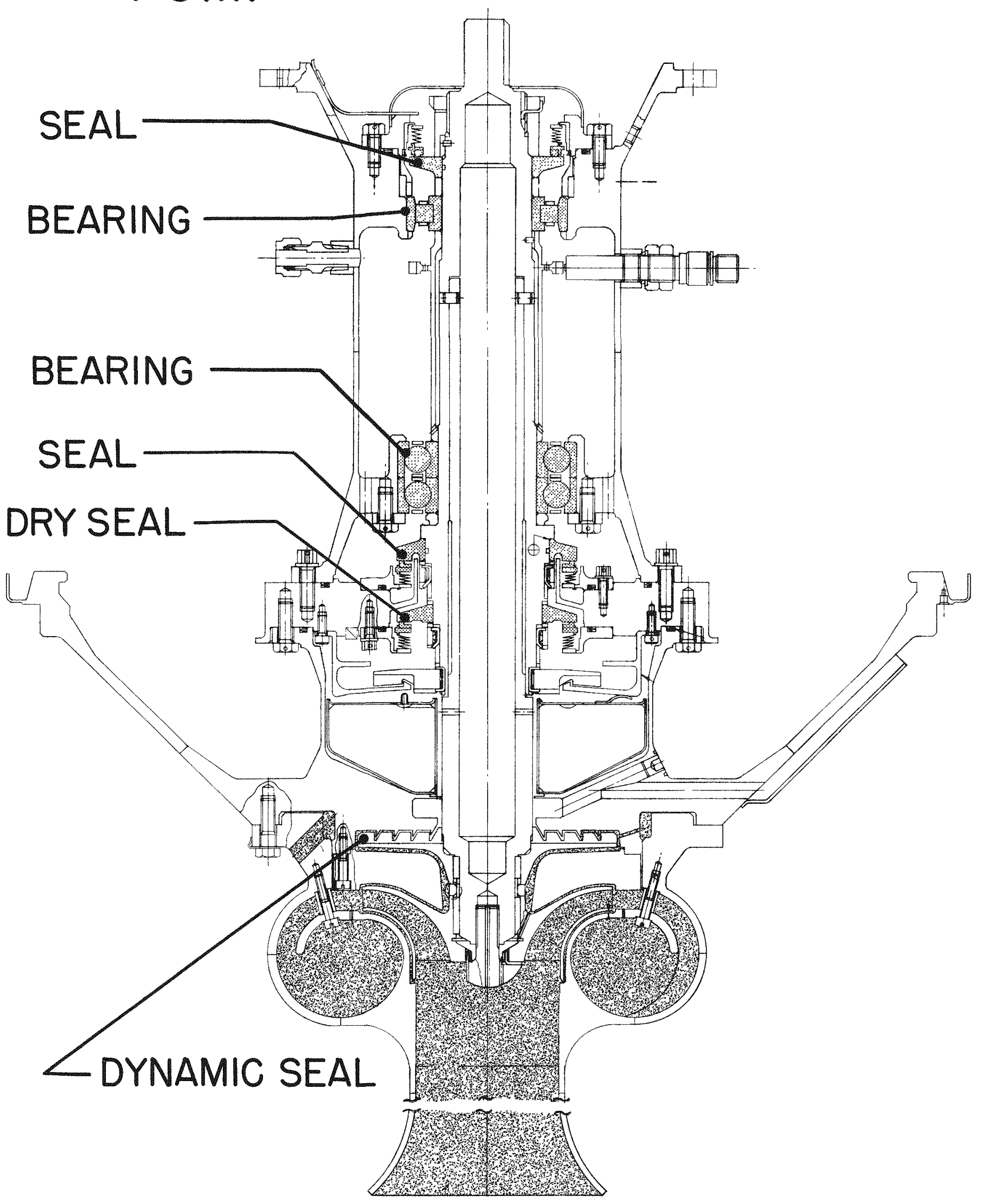




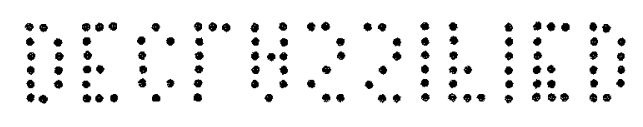
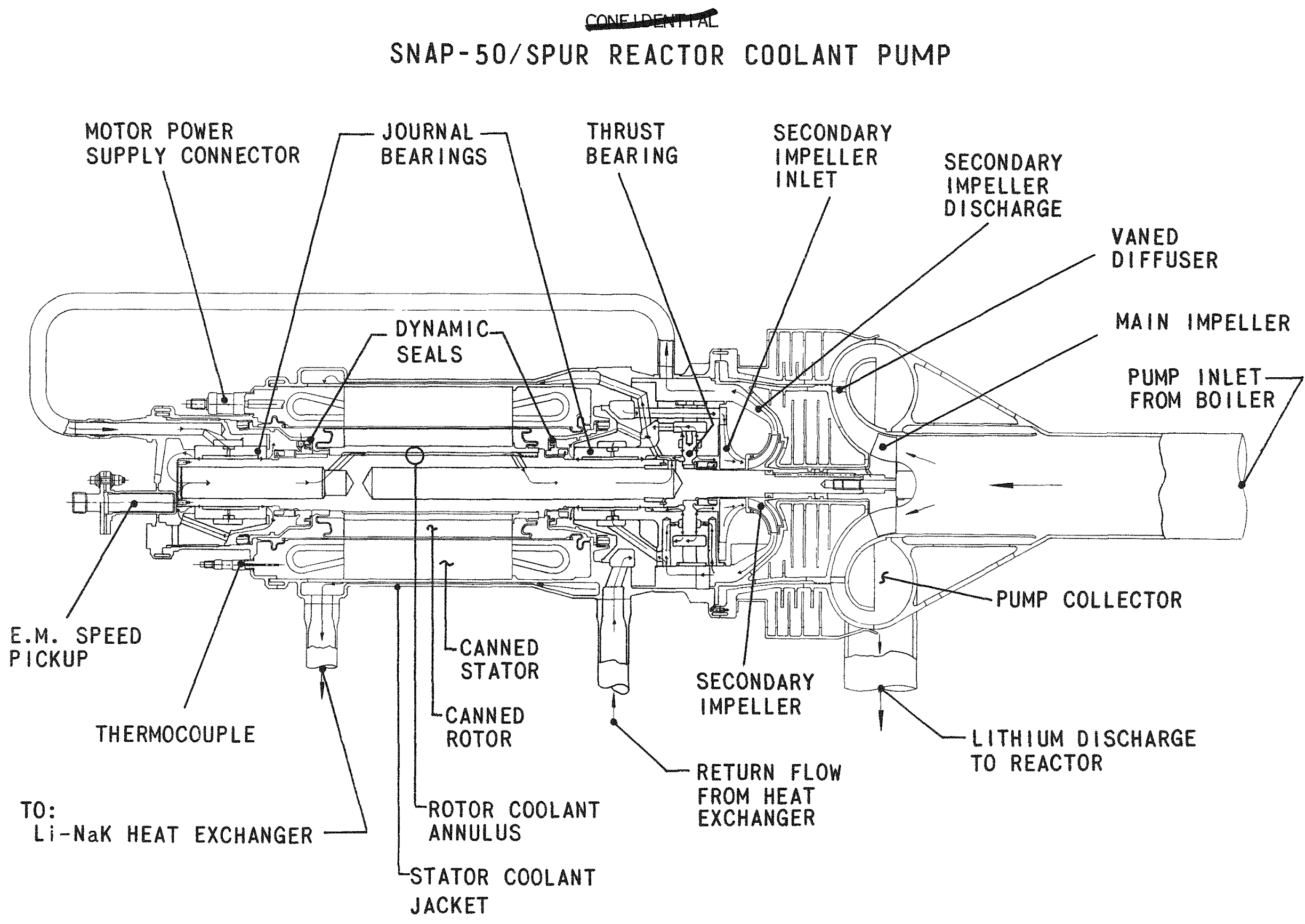

$\therefore \ldots:$

:::..:

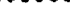

(.....

$\because$

$\therefore$

$\cdots$

.

$\therefore$

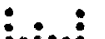

$\therefore: .:$ 


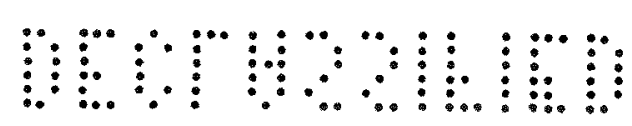

\section{SNAP-50/SPUR CONDENSATE PUMP}

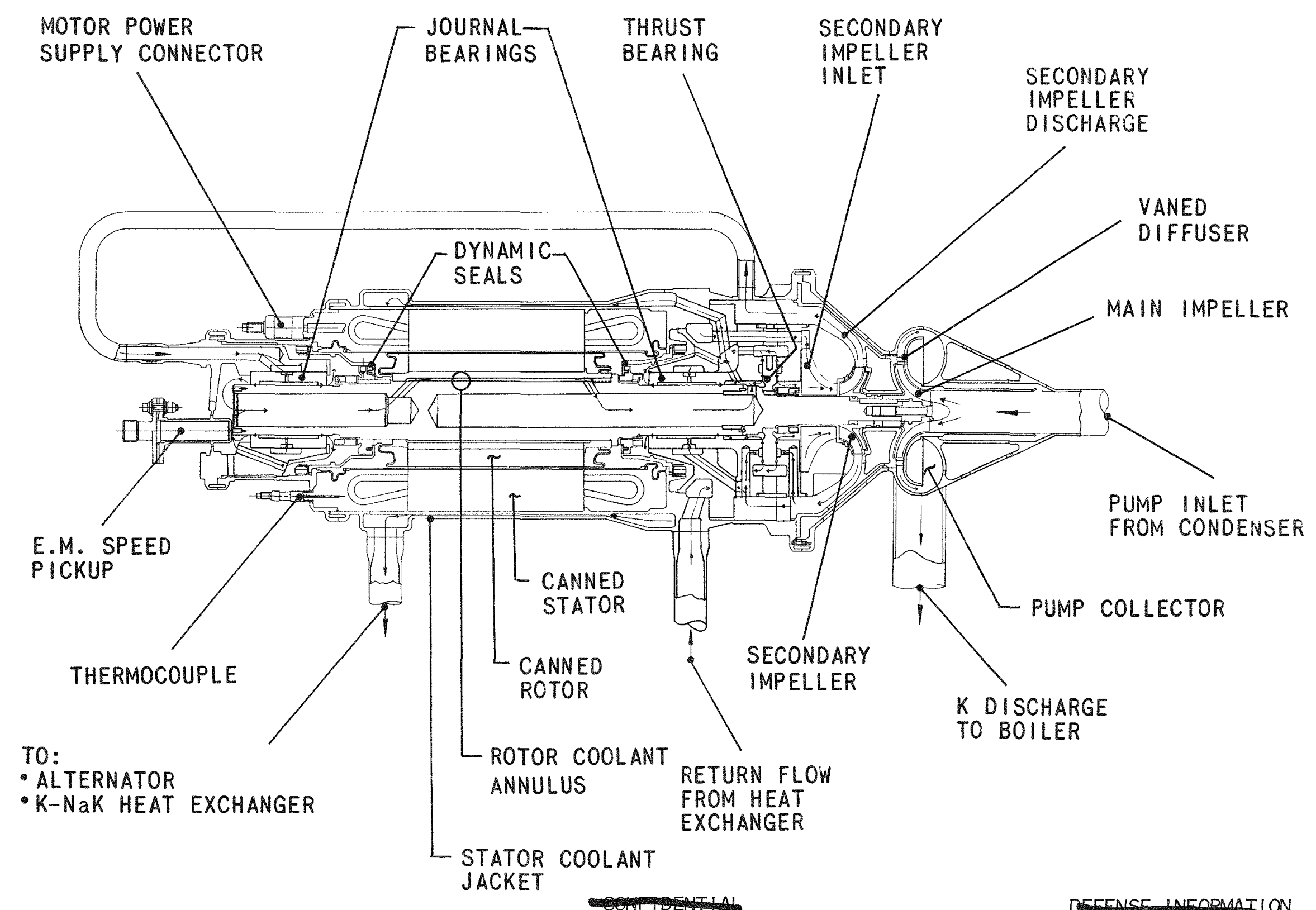

$\ldots$

$:: . .:$

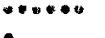

:......

$\because$

$\therefore \because$

$\because$

......

$\therefore \therefore$

:....

$\therefore \cdots:$ 


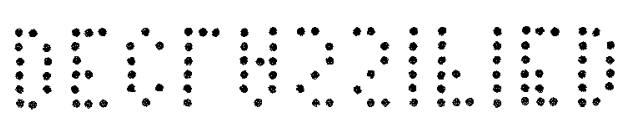
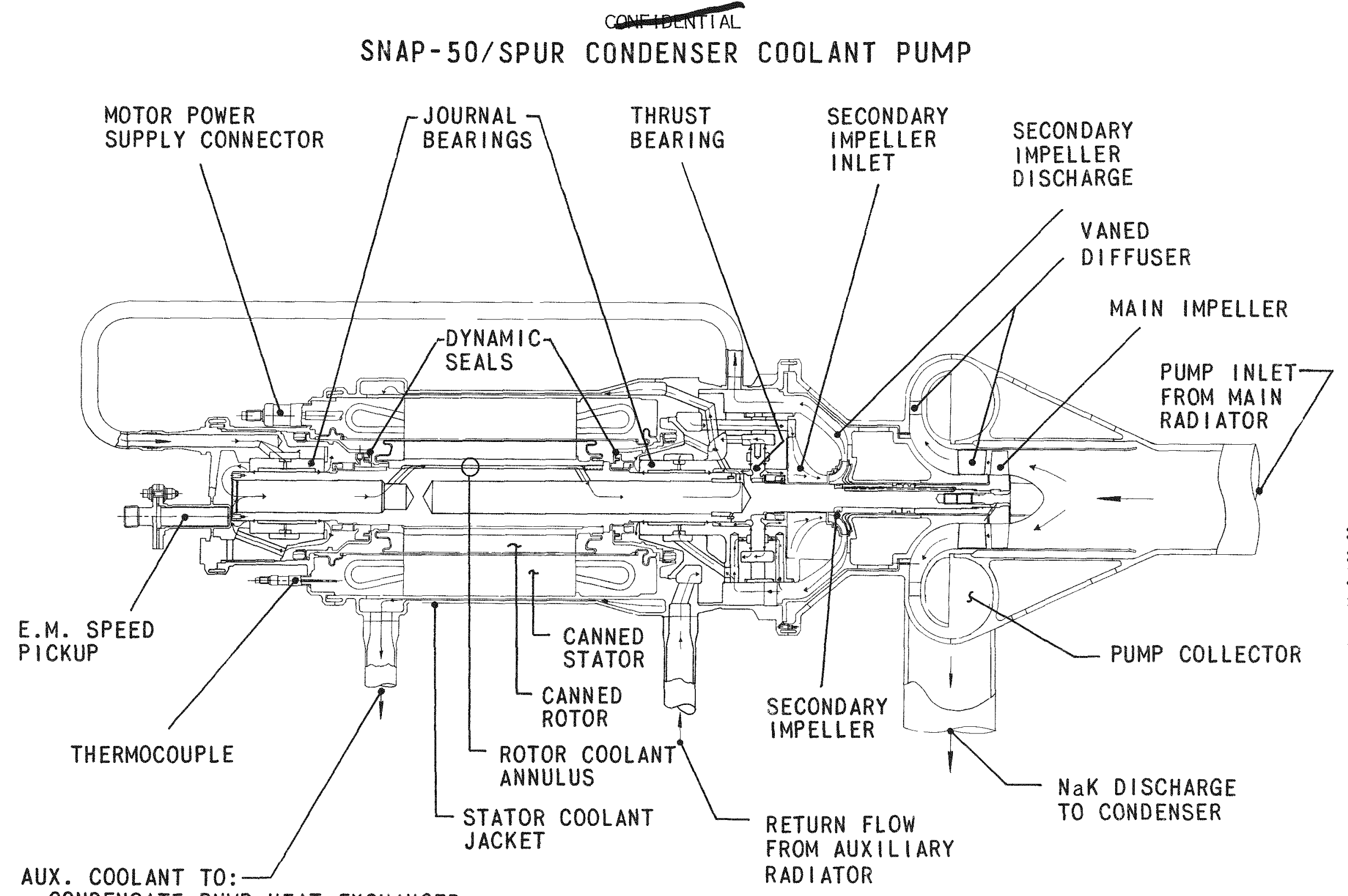

:...:

::i.:

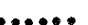

$\because \because:$

$\because$

$\because$

(....:

$\therefore$

$\therefore$ $\therefore \ldots:$

- CONDENSATE PUMP heAT EXCHANGER

RADIATOR

- REACTOR COOLANT PUMP hEAT EXCHANGER

- AUXILIARY RADIATOR 


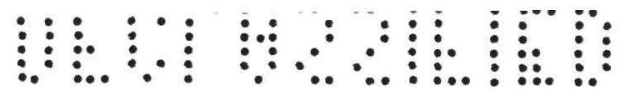

HYDROSTATIC BEARING CONFIGURATIONS

4-POOL, ORIFICE-COMPENSATED

DIA. $=2.375 \mathrm{IN}$.

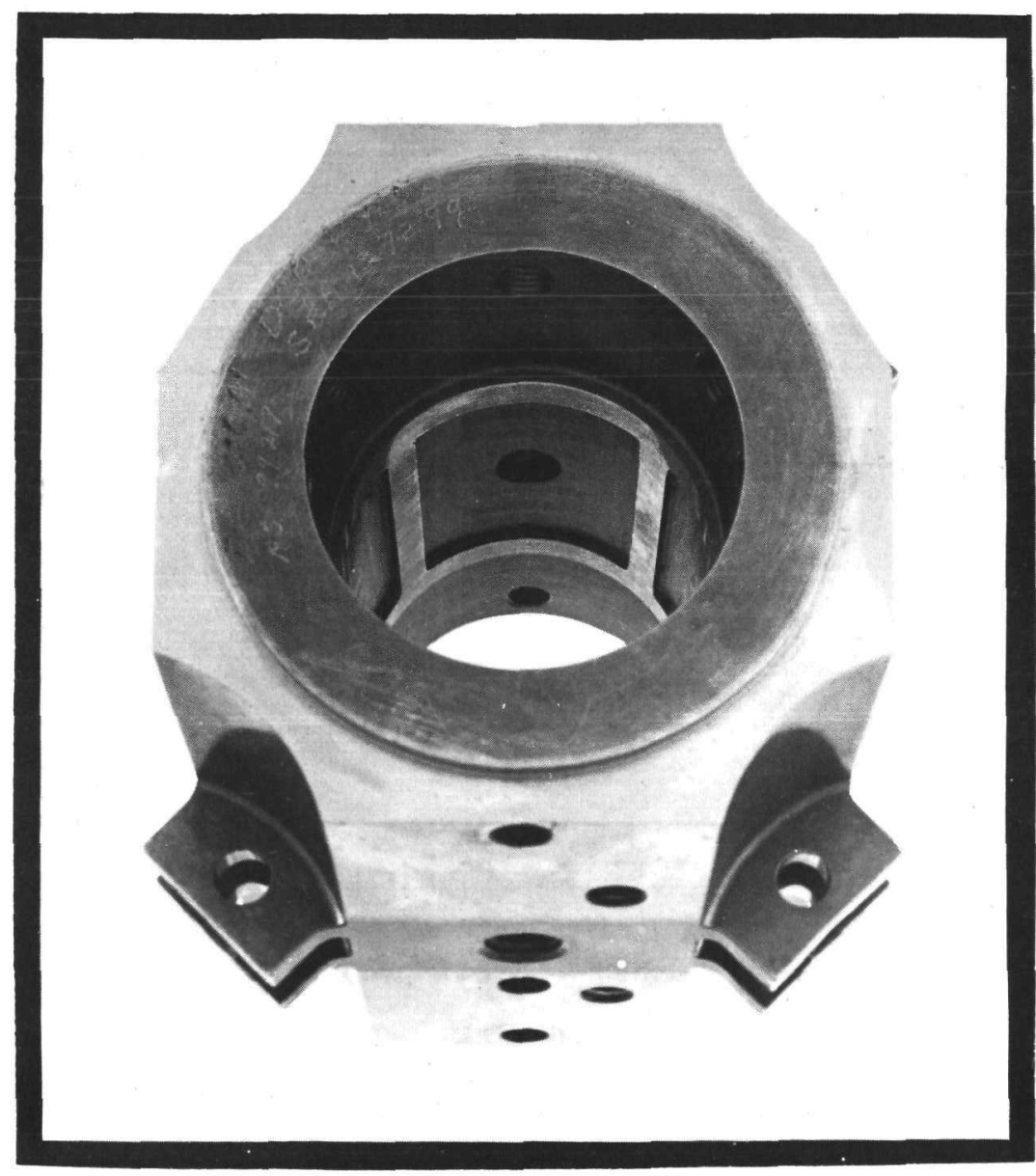

$L / D=1$

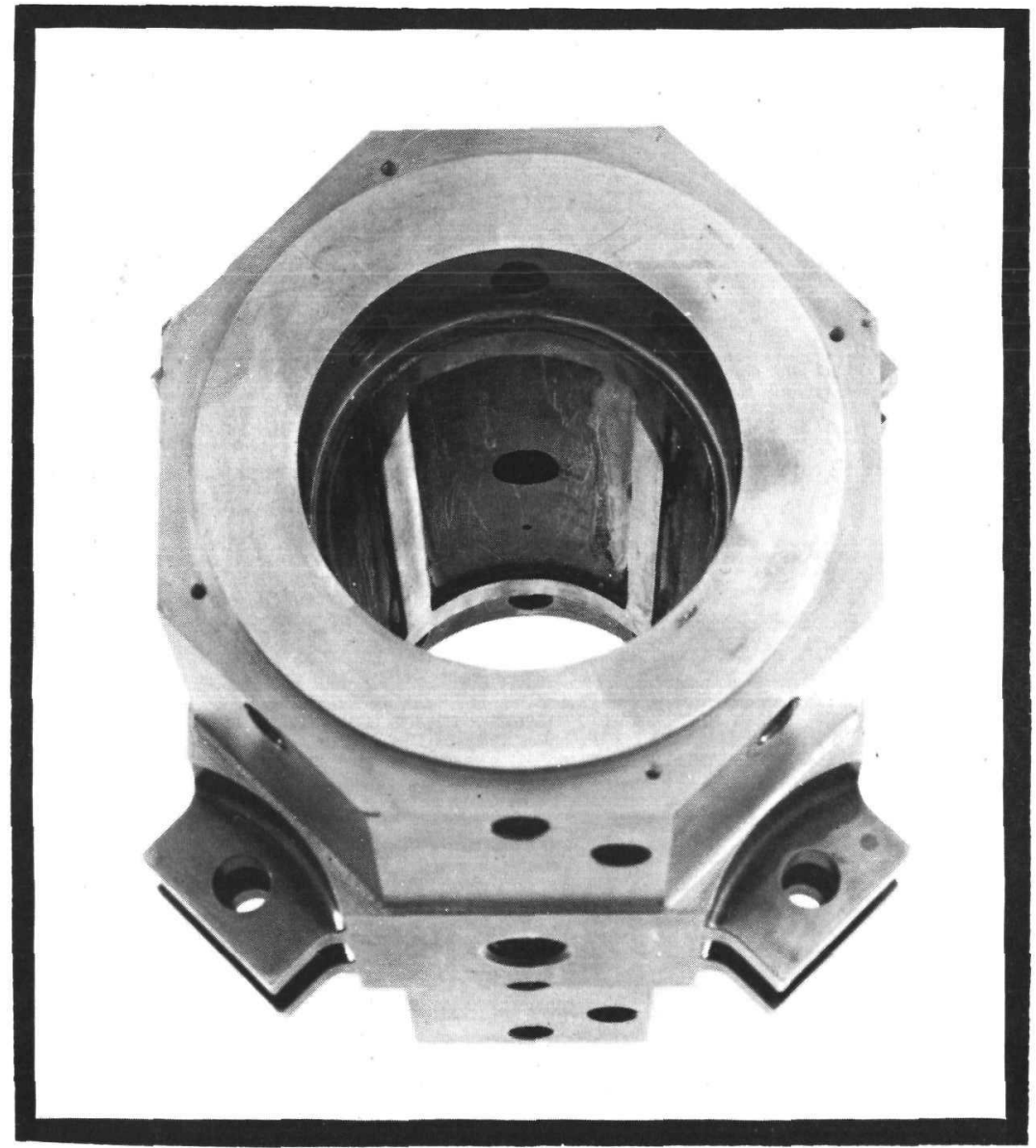

$L / D=1.5$ $\therefore: \cdots:$

:::.::

(n)

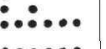

$\because \ldots$

$\therefore \cdots$

$\cdots$

:...:

$\therefore$

$\therefore$

$\therefore \cdots$ 
HYDRODYNAMIC BEARING CONFIGURATION

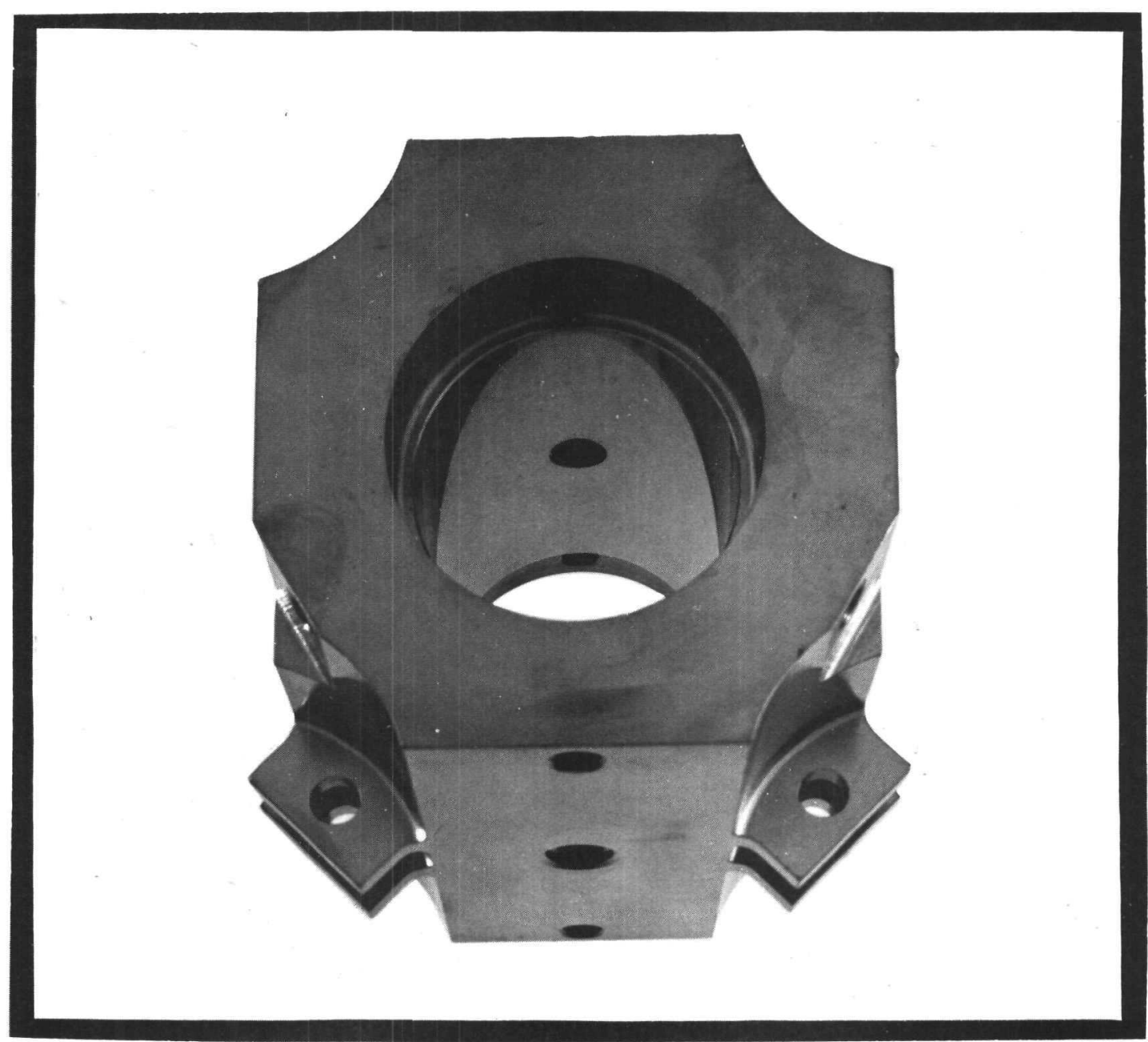

DIA. $=2.375^{\prime \prime}$

$\mathrm{L} / \mathrm{D}=1.5$ 


\section{WATER THRUST BEARING TEST UNIT}

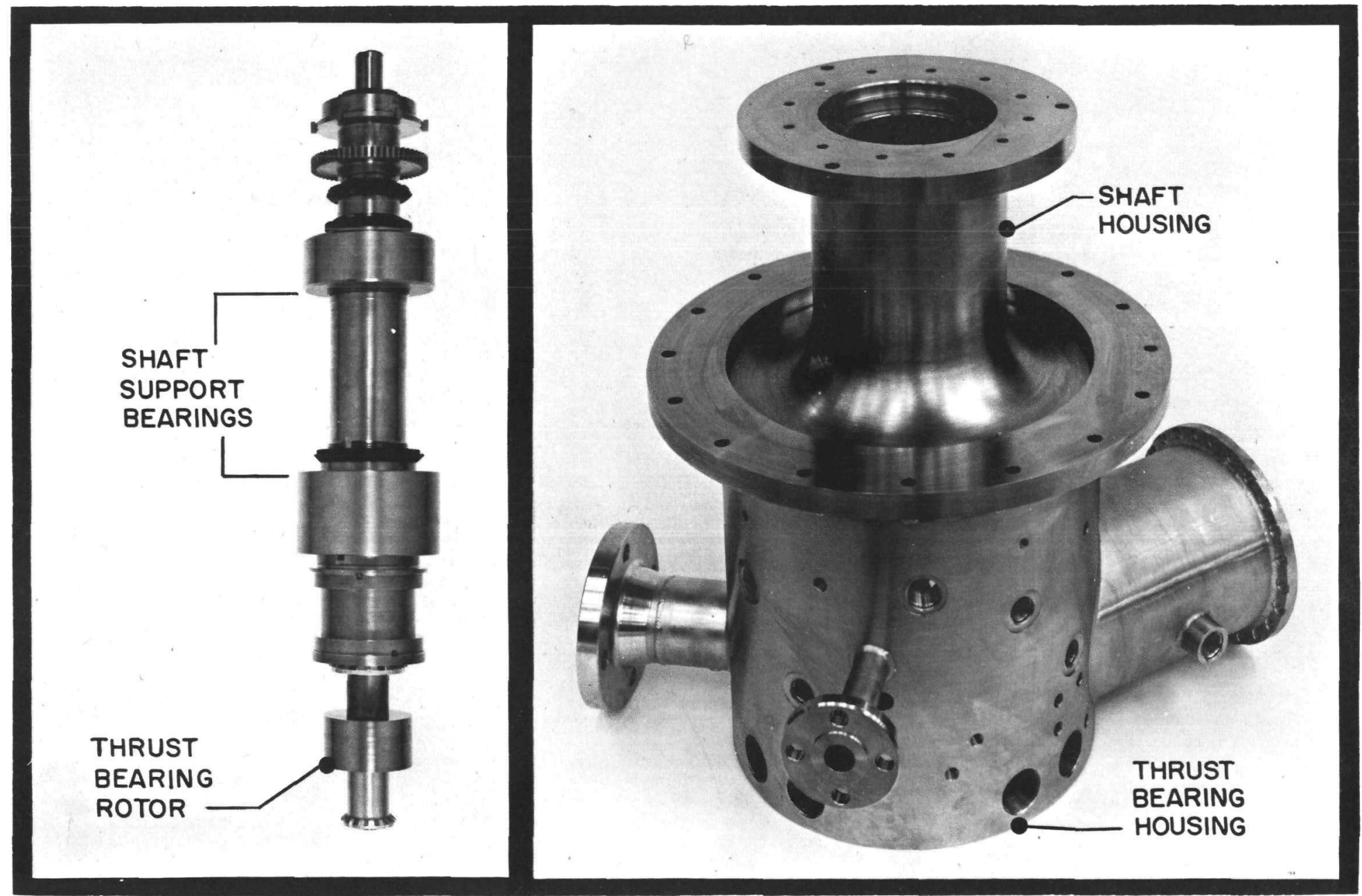

$\therefore: \cdots:$

:::.::

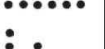

:

$\because \cdots$

$\because \because$

$\cdots$

:

$\because \because$

$\therefore$

$\cdots$

:...: 


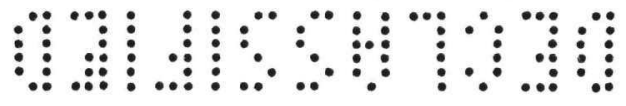

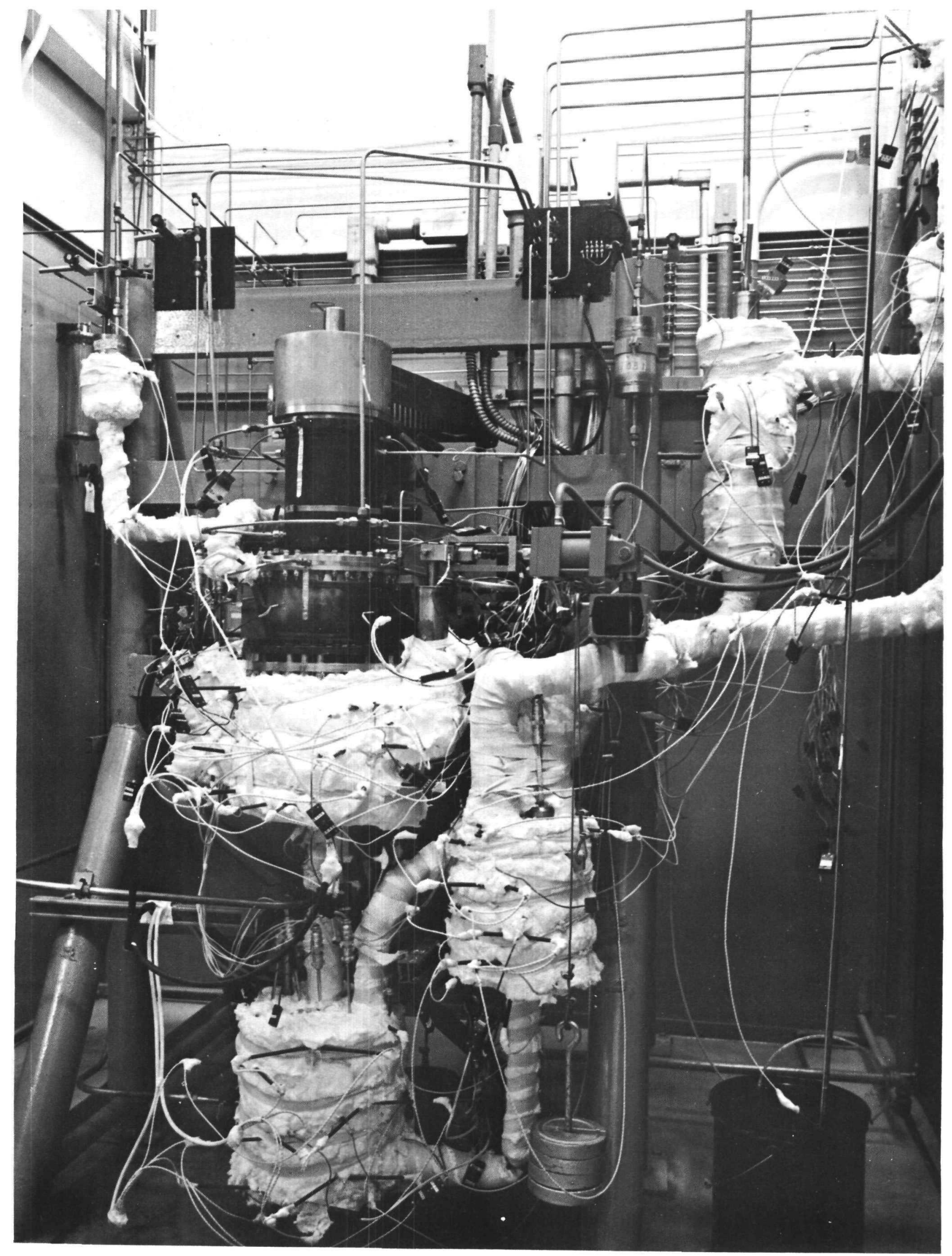


DYNAMIC SEAL IMPELLERS FOR WATER TEST

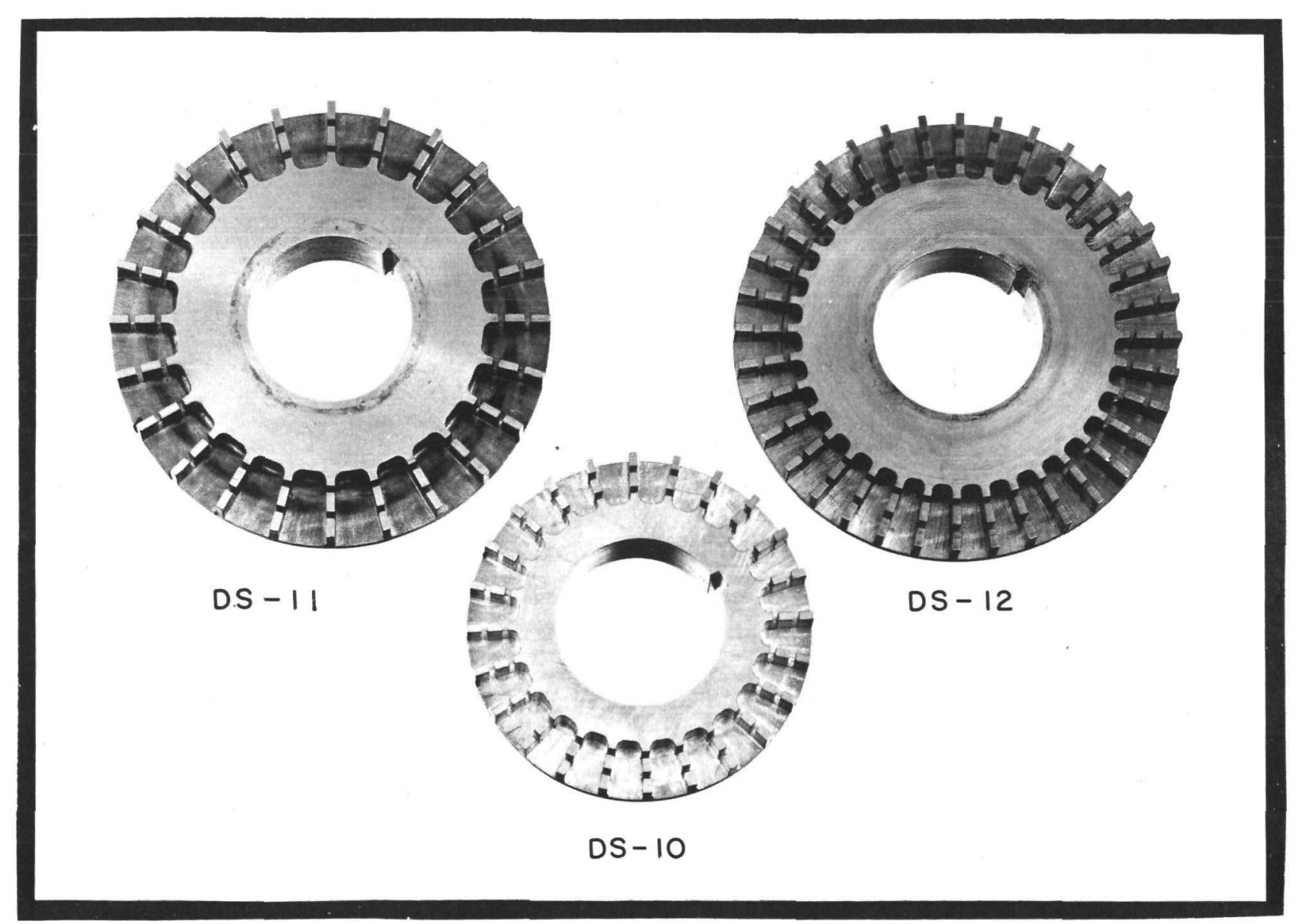

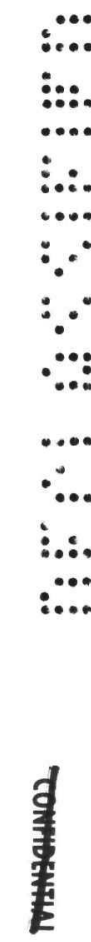

$\ldots$

:

:.....

$\because \cdots$

$\because \because$

$\because$

(n.....

$\therefore$

$\vdots . .$.

$\ldots: . . .:$ 
INTERFACE ON DYNAMIC SEAL

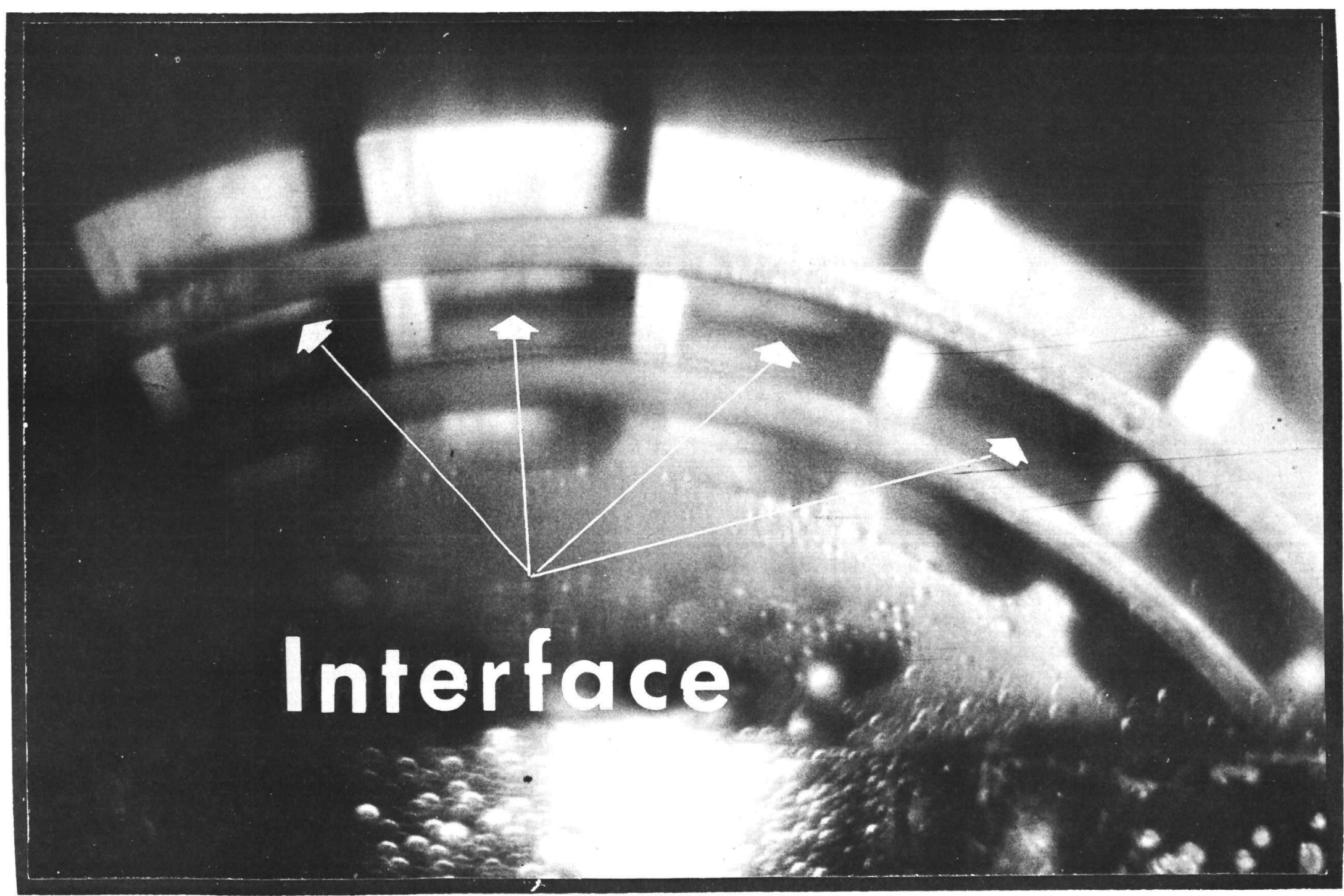

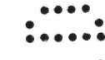

$: \div: .:$

$\vdots . .$.

$\because \cdots$

$\because \because$

$\because \because$

$\because \because$

$\because \cdots$

$\therefore \ldots$

:....

:.... 


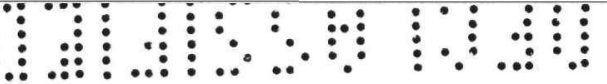

OSCILLOSCOPE TRACES OF SINGLE HYDROSTATIC

WATER BEARING TEST UNIT

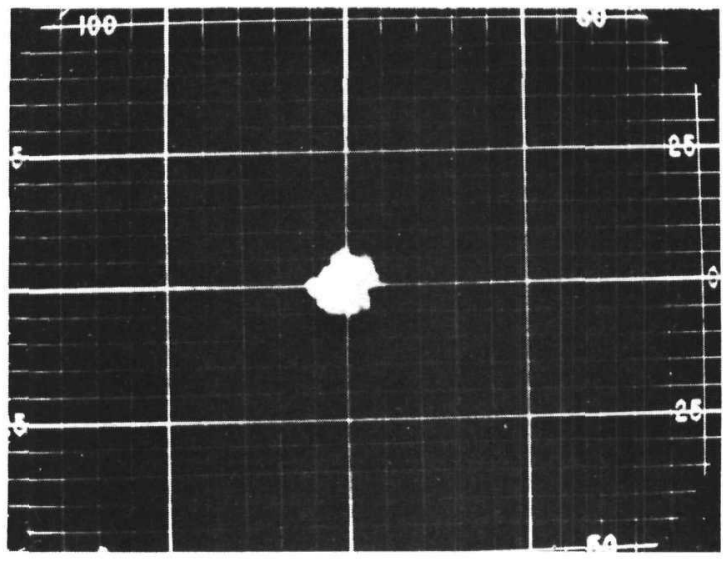

1000 RPM

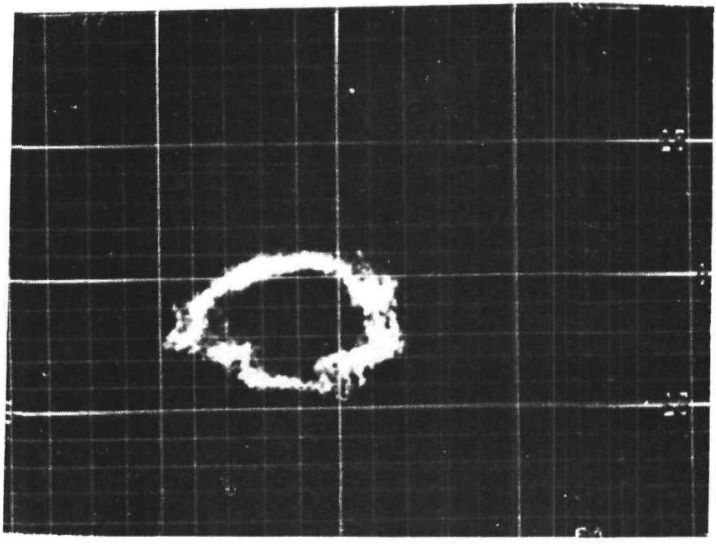

7290 RPM

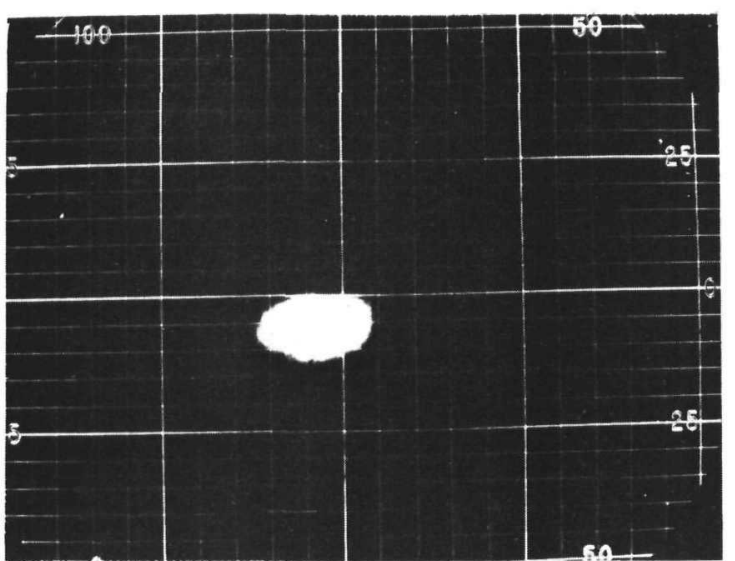

6040 RPM

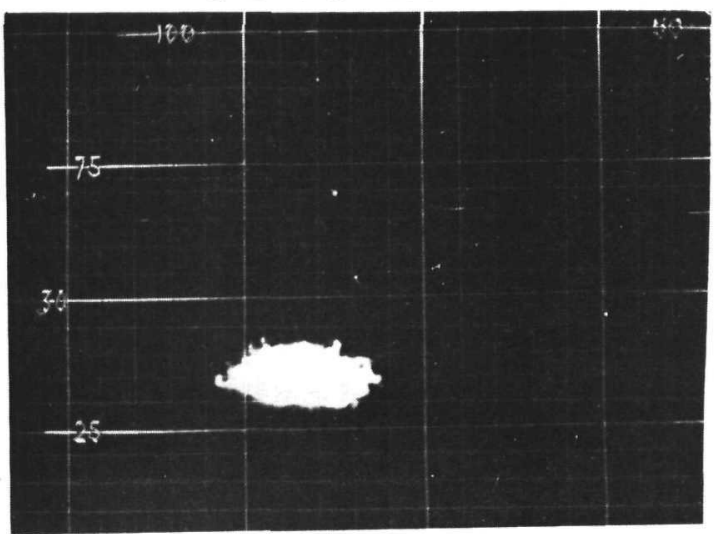

9060 RPM 
(1)

MACHINED IMPELLERS FOR INITIAL REACTOR COOLANT PUMP

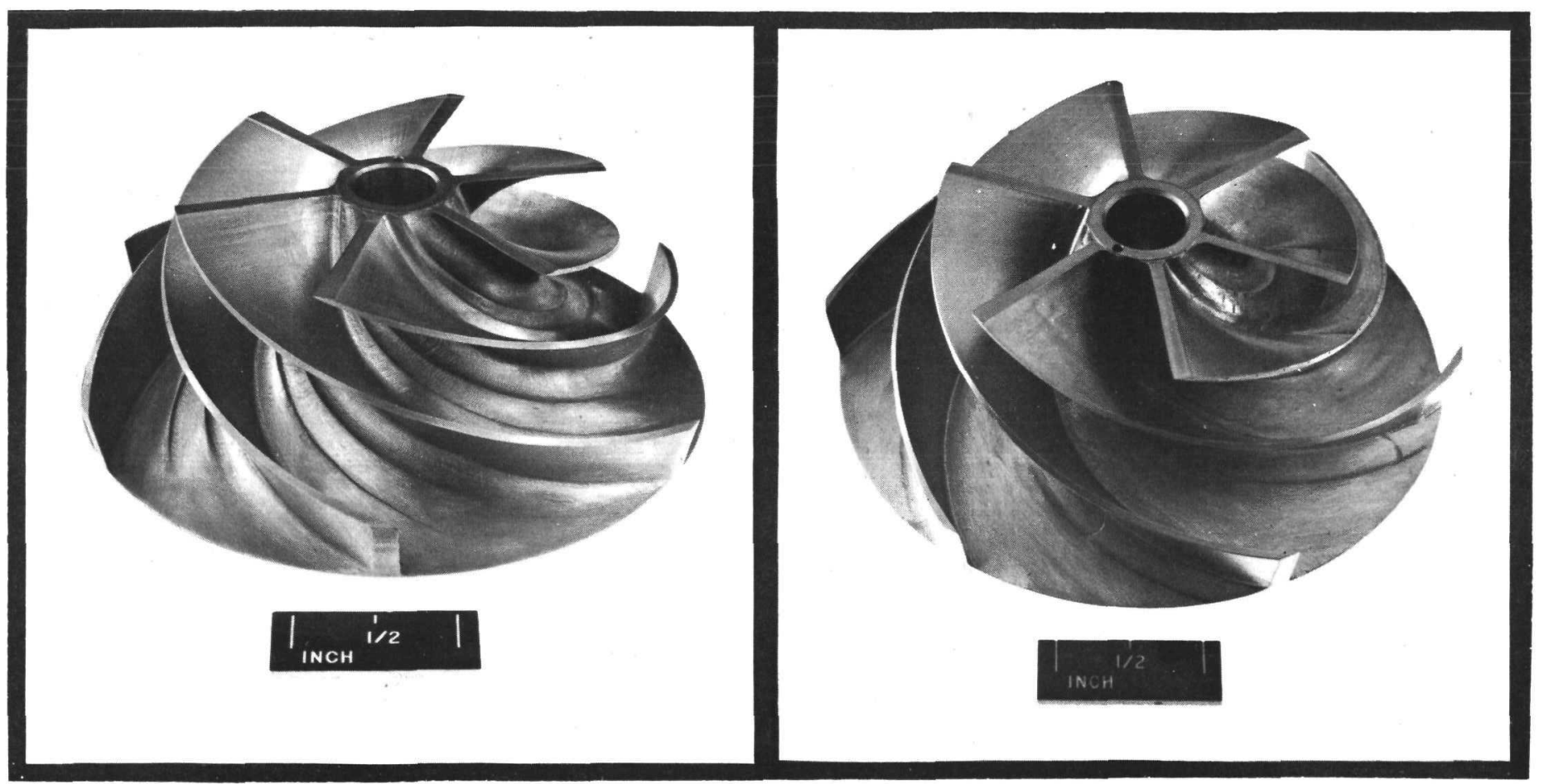




\section{MACHINING OF THE NaK PUMP IMPELLER}

POSITIONING SEQUENCE

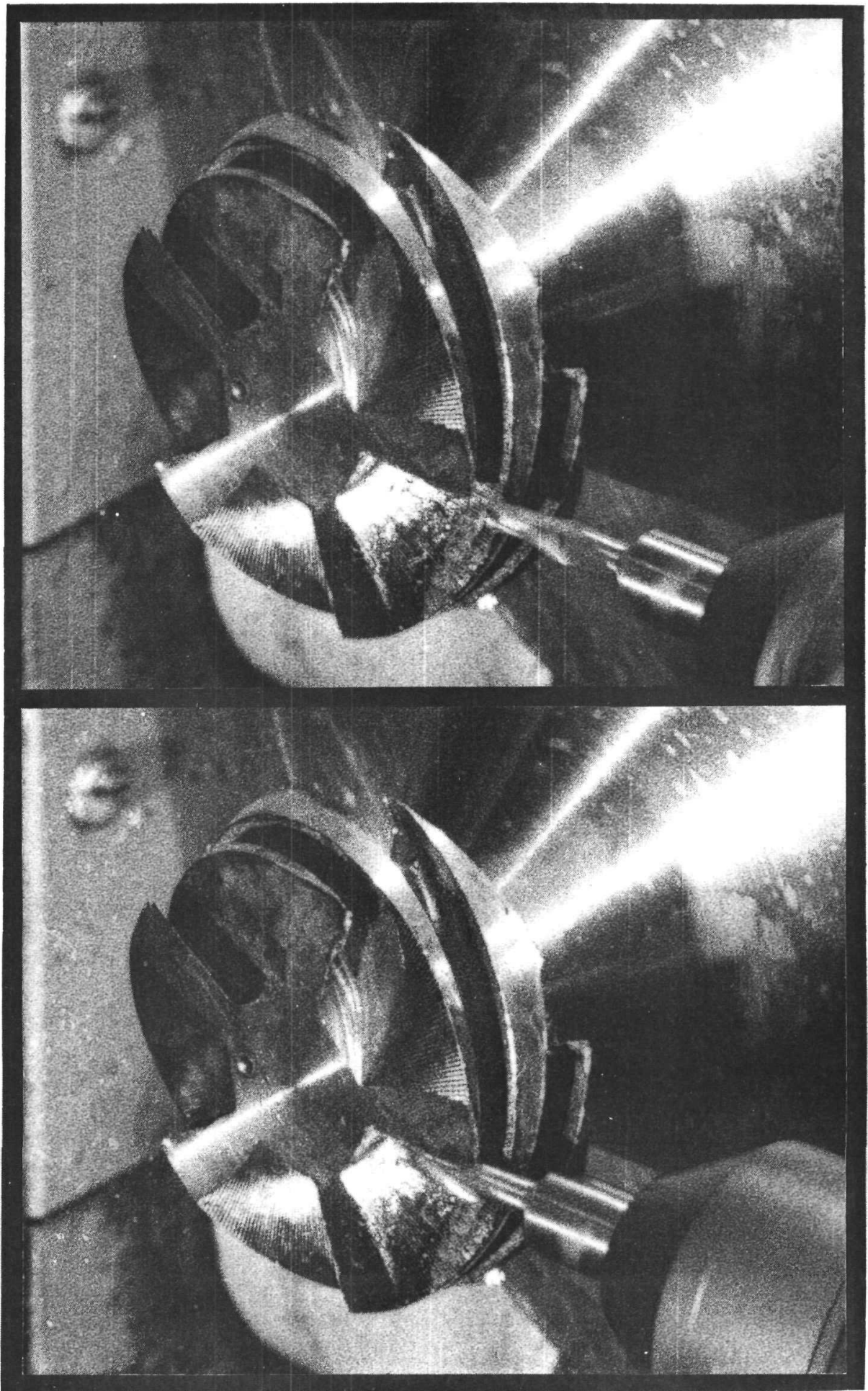

CUTTING SEQUENCE 


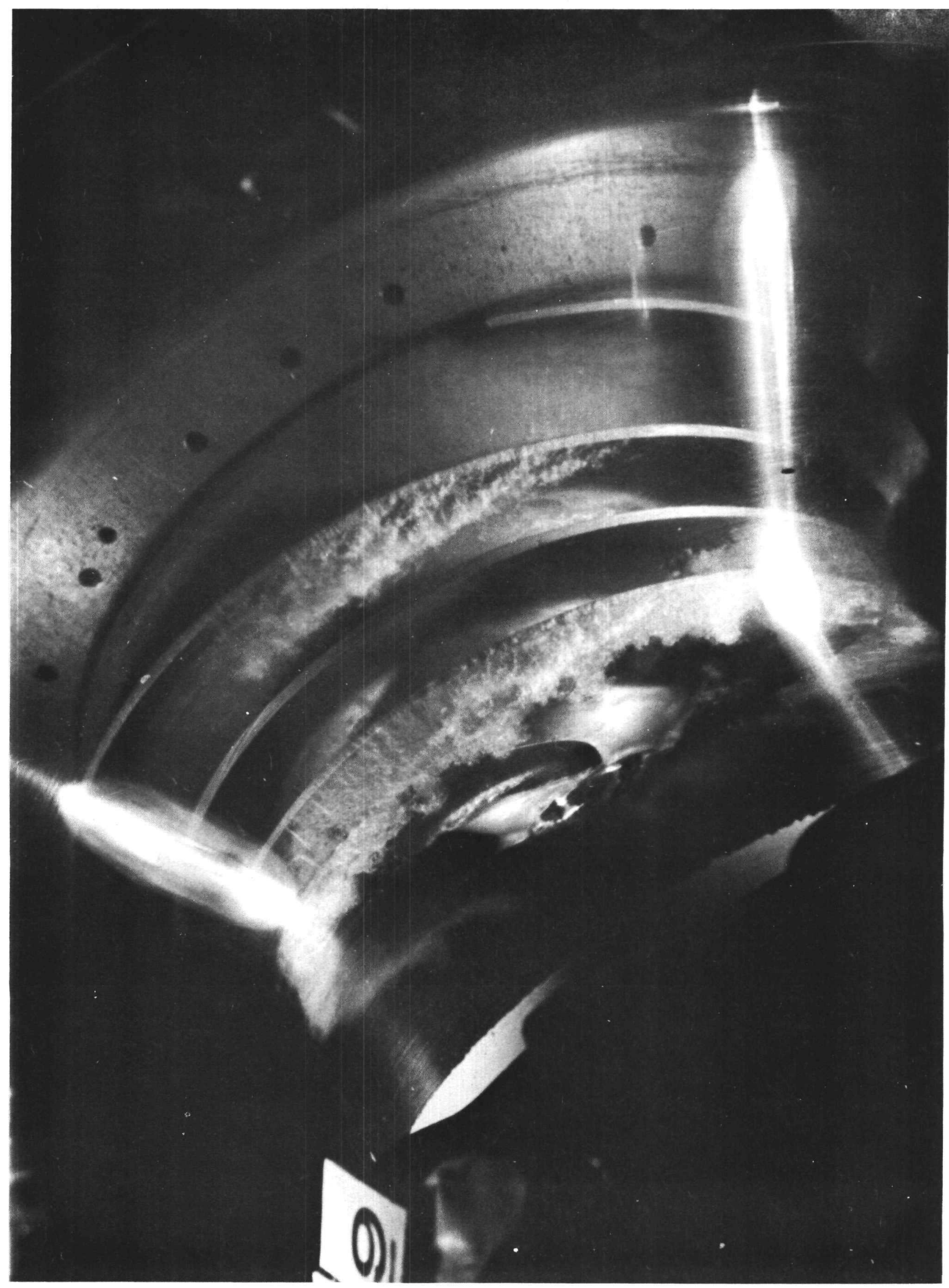

CONFIDENMML 
:

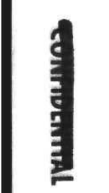

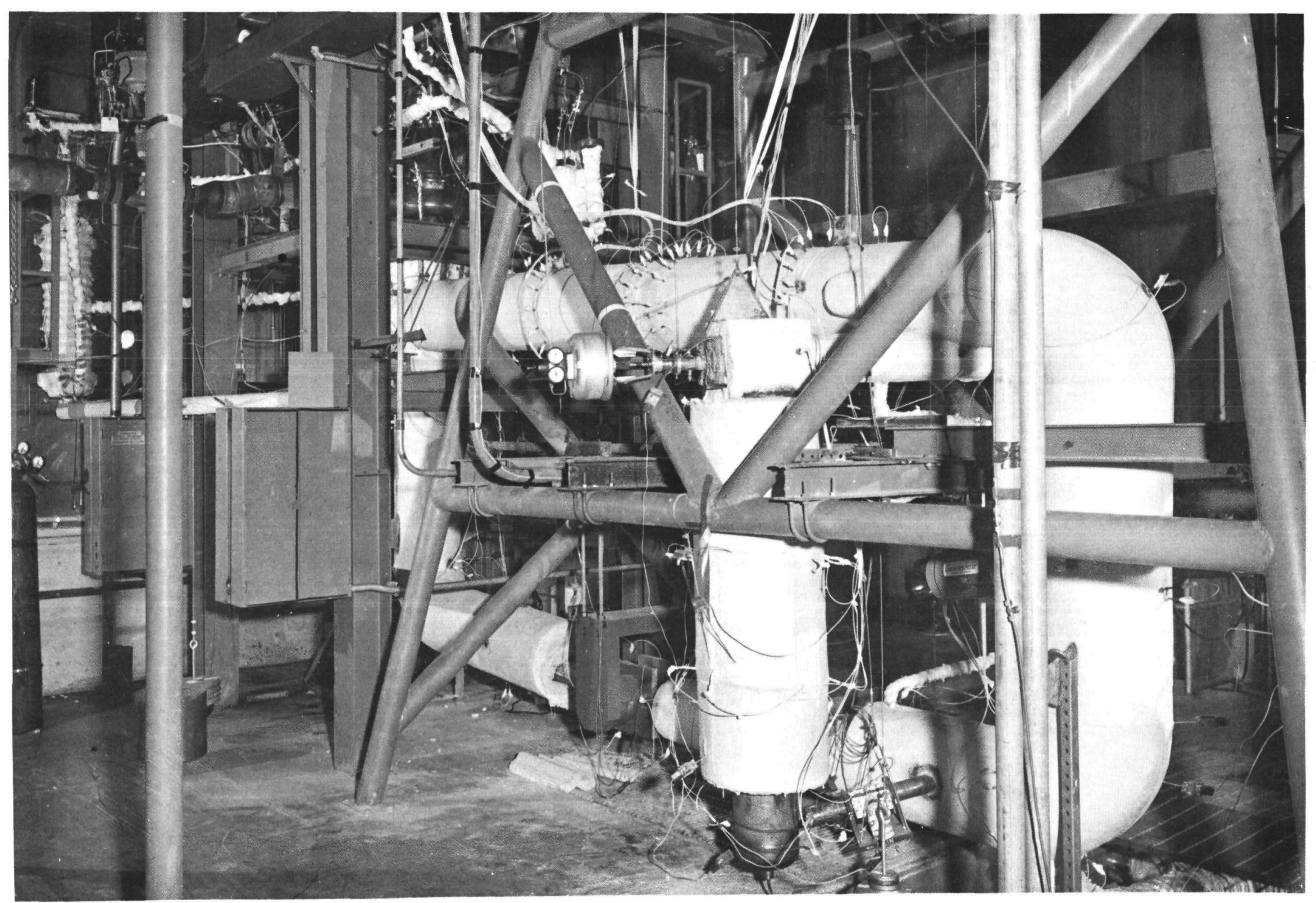

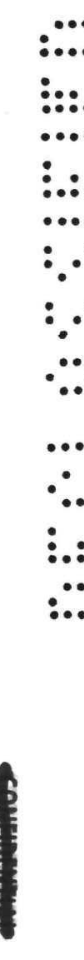

